\title{
SHORT-TERM EFFICACY OF A
}

WORRY POSTPONEMENT INTERVENTION FOR GENERALIZED ANXIETY DISORDER

\author{
by \\ Kathleen Tallon \\ Master of Arts, Ryerson University, 2014 \\ Bachelor of Arts, McGill University, 2012
}

\author{
A dissertation \\ presented to Ryerson University \\ in partial fulfillment of the \\ requirements for the degree of \\ Doctor of Philosophy \\ in the program of \\ Psychology \\ Toronto, Ontario, Canada, 2019 \\ (C) Kathleen Tallon, 2019
}




\section{AUTHOR'S DECLARATION FOR ELECTRONIC SUBMISSION OF A DISSERTATION}

I hereby declare that I am the sole author of this dissertation. This is a true copy of the dissertation, including any required final revisions, as accepted by my examiners.

I authorize Ryerson University to lend this dissertation to other institutions or individuals for the purpose of scholarly research.

I further authorize Ryerson University to reproduce this dissertation by photocopying or by other means, in total or in part, at the request of other institutions or individuals for the purpose of scholarly research.

I understand that my dissertation may be made electronically available to the public. 


\begin{abstract}
Short-Term Efficacy of a
Worry Postponement Intervention for Generalized Anxiety Disorder
\end{abstract}

Doctor of Philosophy, 2019

Kathleen Tallon

Psychology

Ryerson University

\begin{abstract}
Cognitive-Behavioural Therapy (CBT) is an evidence-based treatment for Generalized Anxiety Disorder (GAD). Worry postponement (WP), in which a client is asked to postpone worry until a 30-minute "worry time," is a common component of CBT for GAD; however, the efficacy of WP has never been tested in people with GAD. Further, the mechanisms of change of WP are not known; nor are its effects on cognitive processes and symptoms related to GAD. A better understanding of the efficacy and mechanisms of change of WP could help to optimize CBT for GAD. The goals of the present study were to examine, in a sample of people with GAD, the effects of WP on worry and GAD symptoms, and cognitive processes and symptoms related to GAD. The study also examined the effects of WP on two proposed mediators: stimulus control and metacognitive beliefs. Sixty-seven adults were randomized to one of three conditions: 2week worry postponement intervention (WP), 2-week worry monitoring intervention (MON), or an assessment only control. Participants completed outcome measures before and after the 2week intervention period and at a 2-week follow-up. In the WP and MON conditions, participants completed daily worry monitoring using a phone-based application. All participants showed a significant decrease in past-week worry over the course of the study, with no significant differences between the conditions. There were no significant changes in GAD
\end{abstract}


symptoms across conditions. There was no evidence that WP had superior effects to control groups on cognitive processes or symptoms related to GAD. There was no evidence that stimulus control or metacognitive beliefs mediated the reduction in past week worry in WP. This is the first known study to examine the effects of WP in people with GAD. Whereas worry did decrease on some indices over the course of the study, there were no significant differences between WP and two control conditions. Further this study found no evidence that WP has specific effects on two processes that are thought to be mechanisms of action. The findings of this study demonstrate the need to establish the efficacy of the treatment components used in CBT. 


\section{Acknowledgements}

I am filled with gratitude for the many wonderful people who have contributed to my personal and professional growth, and who have made this dissertation possible.

I would first like to thank my mentor and research supervisor Dr. Naomi Koerner. Naomi, it has truly been a privilege to work with you over the past seven years. You have been an example of professional excellence. Through your guidance you have helped me to challenge myself and work to my full potential. I deeply appreciate the time and consideration you have put into my development. I know I will carry the benefit of your mentorship with me throughout my career.

Thank you to my supervisory committee members. Dr. Martin Antony, your mentorship throughout my graduate training has had a huge influence on me as a clinician and a researcher. I am grateful for the guidance you have given me on my dissertation. Thank you to Dr. Colleen Carney for your insight and feedback throughout the development of this project. Thank you to Dr. David Dozois and Dr. Patrizia Albanese for being a part of my examining committee.

Thank you to my labmates, past and present, whom I have had the pleasure of working alongside. You have been a critical source of support, humour, and encouragement. Thank you to Maya Schonbach for your assistance with data collection for this study.

There are no words to express how much I owe to my parents. Thank you for being an ongoing and unconditional source of support. None of this would be possible without you.

Thank you to my partner Lulu Wei who has helped see me through my PhD by always reminding me what is important and keeping life interesting.

Thank you to the people who volunteered to be research participants in this study.

Finally, thank you to the Canadian Institutes of Health Research for providing research funds for this project. 


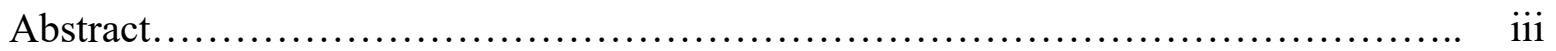

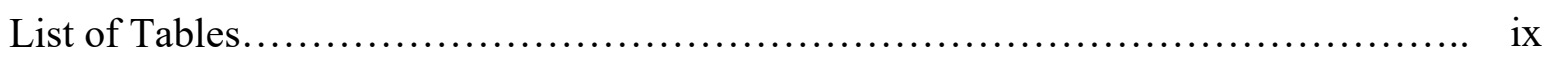

List of Appendices...................................................... xi

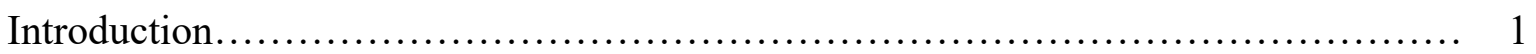

History of Worry Postponement........................................ 3

Stimulus Control Model of Worry Postponement........................... 6

Metacognitive Model of Worry Postponement.............................. 8

Other Approaches to Treating Verbal Behaviour in Psychopathology.............. 10

Evidence for Worry Postponement...................................... 11

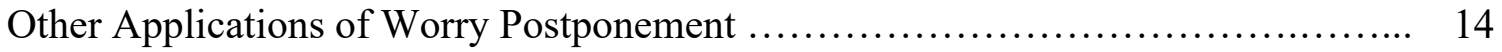

Effects of Worry Postponement on Pathological Worry........................ 19

Proposed Mechanisms of Change in Worry Postponement........................ 20

Proposed Effects of Worry Postponement on Symptoms and Cognitive-Processes

Related to Generalized Anxiety Disorder................................... 22

The Present Study .................................................... 26

Research Questions and Hypotheses..................................... 27

Method................................................................... 31

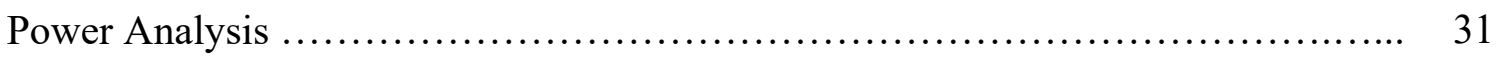

Participants......................................................... 31

Sample Characteristics................................................ 34

Materials.......................................................... 35

Procedure............................................................ 47 
Results.

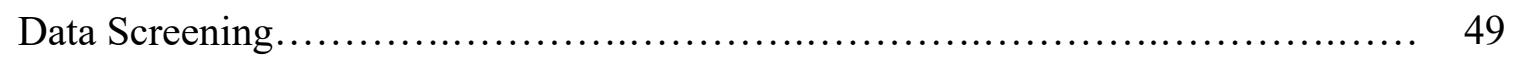

Missing Data........................................................... 49

Compliance to Worry Postponement and Monitoring......................... 51

Baseline Differences Between Groups.................................... 52

Credibility and Expectancy............................................. 52

Main Hypotheses.................................................... 53

Clinically Significant Change............................................ 66

Feedback........................................................... 66

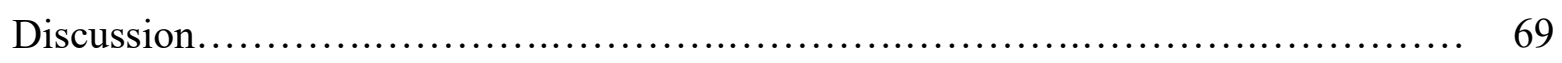

Worry and GAD symptoms........................................... 71

Cognitive Processes Related to GAD ...................................... 71

Stimulus Control.................................................... 73

Proposed Mediators................................................... 74

Symptoms Related to Generalized Anxiety Disorder.......................... 76

Clinically Significant Change............................................ 76

Participant Reactions to the Intervention................................. 77

Explanation of Null Findings.......................................... 77

Worry Diary Responses............................................... 82

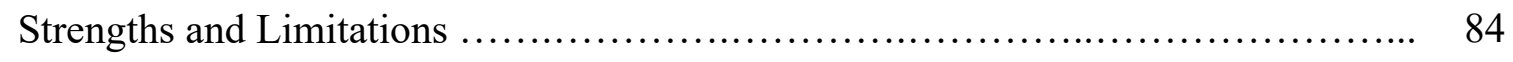

Future Directions........................................................ 86

Conclusions......................................................... 87

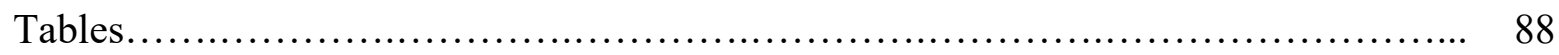


Appendices................................................................ 122

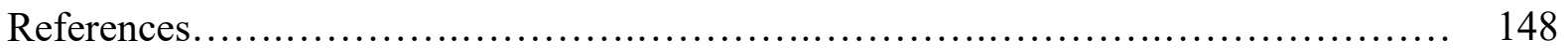




\section{List of Tables}

Table $1 \quad$ Sample Characteristics Separated by Condition............................. 88

Table 2 Internal Consistency for Self-report Measures................................ 89

Table 3 Means and Standard Deviations for Hypothesis 1Questionnaire Measures at Baseline, Postintervention, and Follow-up Separated by Condition.............. 90

Table 4 Compliance to Intervention Instructions in the Worry Postponement and Monitoring Conditions Separated by Condition................................ 92

Table 5 Means and Standard Deviations for Hypothesis 2 Questionnaire Measures at Baseline, Postintervention, and Follow-up Separated by Condition.............. 94

Table 6 Means and Standard Deviations for Hypothesis 2 Behavioural Measures at Baseline, Postintervention, and Follow-up Separated by Condition............. 95

Table 7 Means and Standard Deviations for Hypothesis 4 Measures at Baseline, Postintervention, and Follow-up Separated by Condition........................ 96

Table 8 Means and Standard Deviations for Hypothesis 5 Questionnaire Measures at Baseline, Postintervention, and Follow-up Separated by Condition............. 97

Table 9 Worry Measures from Daily Worry Questionnaire Separated by Condition..... 99

Table $10 \quad$ Between-group Effect Sizes at Postintervention and Follow-up............... 100

Table 11 Correlations Between Baseline Self-Report Worry Measures and Worry Diary Measures in Worry Postponement and Monitoring Conditions $(\mathrm{n}=45) \ldots \ldots \ldots . .102$

Table 12 Hierarchical Linear Models for Self-Reported Worry and Generalized Anxiety Disorder Symptom Measures in all Three Condition (Hypothesis 1)............ 103

Table 13 Hierarchical Linear Models for Self-Reported Worry in Worry Postponement and Monitoring Conditions (Hypothesis 1)................................ 105

Table $14 \quad$ Hierarchical Linear Models for Worry Diary Measures (Hypothesis 1)......... 106

Table $15 \quad$ Hierarchical Linear Models for Mindfulness Measures (Hypothesis 2)......... 107

Table 16 Hierarchical Linear Models for Problem Solving and Cognitive Avoidance Measures (Hypothesis 2)................................................. 108

Table 17 Hierarchical Linear Models for Attentional Control Measures (Hypothesis 2).. 111

Table 18 Hierarchical Linear Models for Frequency of Worry Episodes (Hypothesis 3).. 114 
Table 19 Hierarchical Linear Models for Proposed Mediator Variables (Hypothesis 4).. 115

Table $20 \quad$ Hierarchical Linear Models for Symptom Measures (Hypothesis 5)........... 117

Table 21 Percentage of Participants Achieving Clinically Significant Change on Symptom Measures Separated by Condition.......................... 120

Table 22 Participant Feedback on Worry Postponement and Monitoring Interventions

Separated by Condition................................................. 


\section{List of Appendices}

Appendix A Participant Flow Diagram......................................... 122

Appendix B Participant Home Screen of MovisensXS Application.................. 123

Appendix C "Record Your Worry" Form..................................... 124

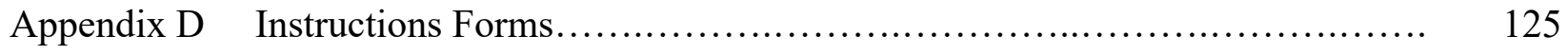

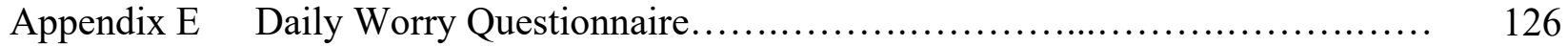

Appendix F Daily Worry Postponement Questionnaire........................... 127

Appendix G Night Worry Questionnaire ................................... 128

Appendix H Worry Postponement Instructions................................... 129

Appendix I $\quad$ Worry Monitoring Instructions.................................. 130

Appendix $\mathbf{J} \quad$ Instructions for Completing Phone Forms: Worry Postponement Condition............................................................ 131

Appendix K Instructions for Completing Phone Forms: Worry Monitoring Condition... 134

Appendix L Worry Postponement Feedback Questionnaire......................... 137

Appendix M Worry Tracking Feedback Questionnaire............................ 138

Appendix N Qualitative Feedback on Worry Postponement Intervention $(n=23) \ldots \ldots \ldots \quad 139$

Appendix O Qualitative Feedback on Monitoring Intervention $(n=21) \ldots \ldots \ldots \ldots \ldots \ldots . .144$ 
Short-Term Efficacy of a Worry Postponement Intervention for Generalized Anxiety Disorder Worry has been defined as a negative, repetitive, future-oriented thought process that typically concerns a problem whose outcome is uncertain (Borkovec, Robinson, Pruzinsky, \& DePree, 1983). Whereas most people worry to some degree (Tallis, Davey, \& Capuzzo, 1994), very frequent and pervasive worry can lead to significant interference in a person's life in the form of generalized anxiety disorder (GAD). Individuals with GAD report experiencing their worry as excessive and uncontrollable (American Psychiatric Association, 2013). Worry is proposed to underlie many of the difficulties associated with GAD including cognitive complaints and sleep difficulties (Borkovec, Ray, \& Stöber, 1998). Worry has also been linked to health complaints (Brosschot, Gerin, \& Thayer, 2006). Worry is proposed to prolong the stress response, which can lead to physiological changes such as reduced immune functioning, reduced heart rate variability, and increased blood pressure (Brosschot et al., 2006). Individuals with GAD report greater use of primary care and specialized health resources than do individuals with other anxiety disorders (Wittchen, 2002). Worry has been a central focus of research on GAD and the main target of psychological treatment for this disorder.

Cognitive-behavioural therapy (CBT) is an efficacious treatment for GAD (Borkovec \& Costello, 1993; Covin, Ouimet, Seeds, \& Dozois, 2008; Dugas et al., 2003; Dugas et al., 2010; Ladouceur et al., 2000; Wetherell, Gatz, \& Craske, 2003). However, a significant proportion of people with GAD do not remit following CBT. According to two meta-analyses, 12 months after completing treatment only between 39 to $57 \%$ of people who received CBT were classified as recovered from GAD (Fisher, 2006; Hanrahan, Field, Jones, \& Davey, 2013). This points to a need to strengthen existing treatments. 
CBT for GAD is a multicomponent treatment. The effects of single components of CBT for GAD have seldom been studied. By examining the efficacy of single components of CBT for GAD, the most powerful components of the treatment can be selected to optimize treatment packages and improve patient response. If single component treatments are found to lead to clinically meaningful effects, they can be easily disseminated and administered with minimal additional training. Further to the lack of research on components of CBT, little is known about the mechanisms of change in CBT for GAD (Covin et al., 2008). Identifying mechanisms of change can further help to tailor treatment and inform models of pathological worry.

Worry postponement (also known as stimulus control) is a component of several CBT protocols for GAD (Borkovec, 2006; Wells, 2006; Newman \& Borkovec, 2002; Rygh \& Sanderson, 2004). In worry postponement, worry is treated as a maladaptive coping behaviour. This intervention aims to reduce the frequency and generalization of worry by asking individuals to notice incidents of naturally occurring worry, and to then disengage from and defer their worry to a designated 30-minute "worry time" later in the day (Borkovec, Wilkinson, Folensbee, \& Lerman, 1983). Worry postponement is widely recommended. In a survey of clinicians practicing CBT with people with GAD, $65 \%$ endorsed using "stimulus control for worry" as a treatment technique, suggesting that the technique is frequently used in clinical practice (Szkodny, Newman, \& Goldfried, 2014).

Instructions and rationale for worry postponement's use vary across sources. Variability in rationale and instructions suggest that little is known about how the intervention works or what the optimal way to deliver it is. No research has been done to test the proposed mechanisms of change in worry postponement. Further, very little research has been done on the efficacy of worry postponement for reducing worry, and to my knowledge, this intervention has never been 
studied in a sample of people with GAD, the population it is intended for. The present study begins to address the above issues by examining the short-term efficacy of worry postponement as an intervention for worry in GAD and by attempting to elucidate mediators of change in this intervention.

\section{History of Worry Postponement}

Worry postponement was adapted from a behavioural intervention for insomnia called stimulus control (Borkovec, Wilkinson, et al., 1983). Understanding the development of stimulus control for insomnia informs the understanding of its application to pathological worry. According to behaviour theory, stimulus control happens when the probability of a behaviour occurring is dependent on what cues are present. Stimulus control may involve stimulus discrimination, in which different stimuli cue different behaviours. For example, when looking at a traffic light, a driver learns to stop at a red light, and to go at a green light. Stimulus control also involves stimulus generalization, in which various stimuli cue one behaviour. For example a driver learns to stop at a red light, a stop sign, or at a traffic cop's hand signal. When a driver has learned to stop their car according to the presence or absence of various stimuli, the behaviour can be said to be under stimulus control.

Stimulus control treatment for insomnia is based on the rationale that the behaviour of people with insomnia leads them to associate stimuli related to bed (e.g., the bedroom) with wakefulness (Bootzin, 1972). In other words, stimuli that should cue sleep (e.g., nighttime, the bed) have become associated with wakefulness. Stimulus control treatment is intended to change this association so that the person with insomnia comes to associate bed-related stimuli with sleepiness and sleep onset. The intervention is carried out through five instructions: 1) go to bed only when sleepy, 2) use the bedroom only for sleeping, 3) get out of bed if you are unable to 
sleep, 4) get out of bed at the same time every morning, 5) do not nap (Bootzin, 1972). Stimulus control for insomnia has been widely studied and has been shown to be efficacious on its own and as part of a treatment package (Morin et al., 1999). When delivered on its own, stimulus control for insomnia is typically taught by a therapist over four weekly sessions. Additionally, participants practice the stimulus control instructions on their own on a daily basis, in between sessions with the therapist (Morin, Culbert, \& Schwartz, 1994). Weekly sessions with the therapist involve delivering the treatment rationale and instructions, reviewing records of daily stimulus control practice, and troubleshooting any problems that have arisen in the application of the instructions (Lacks, Bertelson, Gans, \& Kunkel, 1983). Stimulus control has been delivered in individual, group, and self-administered forms (e.g., delivered via written instructions with no therapist contact; Morin et al., 1999). One meta-analysis examined the effect of stimulus control, delivered variously in group, individual, and self-guided formats (Morin et al., 1994). Stimulus control on its own was shown to have a large effect size, compared to control conditions, for reducing sleep-onset latency $(d=0.81)$ and for reducing time awake after sleep onset $(d=0.70$; Morin et al., 1994). One study of stimulus control administered individually found that gains from treatment were maintained at a 6-month follow-up (Baillargeon, Demers, \& Ladouceur, 1998). Self-guided stimulus control has been shown to be efficacious. Morawetz (1989) found that stimulus control delivered via written manual and audiotapes was comparably efficacious to therapist-guided treatment ( $48 \%$ reduction in sleep onset latency compared to $50 \%$ respectively) for unmedicated participants with insomnia. In head-to-head comparisons with other active treatments, eight weekly sessions of stimulus control was superior to relaxation for improving sleep onset latency (between-group $d=0.93$; Espie, Lindsay, Brooks, Hood, \& Turvey, 1989), 
and four weekly sessions of stimulus control was found to be superior to imagery training for improving sleep maintenance (between-group $d=0.46$; Morin \& Azrin, 1988).

Stimulus control is recommended by the American Academy of Sleep Medicine as an evidence-based treatment for insomnia (Chesson et al., 1999). There is some evidence that multicomponent insomnia treatment including stimulus control leads to improvement in symptoms of depression (pre to posttreatment $d=1.49$ on Beck Depression Inventory), state anxiety $(d=0.92)$, and trait anxiety $(d=1.55$; Jacobs, Benson, \& Friedman, 1993). Depression and anxiety have not been widely studied as outcomes of stimulus control treatment for insomnia. Another advantage of stimulus control for insomnia is that it is a brief effective treatment that can be delivered by healthcare workers with minimal additional training (Baillargeon et al., 1998).

Some research has examined the factors that moderate outcome in stimulus control for insomnia. An early study examined variation in the instructions for stimulus control. In one condition labelled "counter control" participants were instructed, if they were unable to sleep, to sit up in bed and engage in another activity until they were sleepy (Zwart \& Lisman, 1979). The counter control instructions are in contrast to the original instructions in which people are told to leave the bed if they cannot sleep. Treatments were delivered over the course of four, weekly 30 minute sessions. Participants followed instructions daily on their own. The counter control condition and original stimulus control instructions led to comparable reductions in sleep onset latency from pretreatment to 4 -week follow-up ( $d \mathrm{~s}=1.77$ and 1.35 respectively). Given that both the counter control and stimulus control instructions led to large effects on sleep onset, the authors concluded that the efficacy of stimulus control may be due to "its ability to contingently disrupt sleep-incompatible activities and/or cognitions" rather than achieving stimulus control 
per se (Zwart \& Lisman, 1979, p. 117). In other words, the key element in the intervention may be training people's attention to notice when they are engaging in a sleep-incompatible behaviour (e.g., lying in bed worrying), stopping the behaviour, and then doing something different.

Taken together, the research on stimulus control suggests that it is a highly efficacious treatment for insomnia that may also have effects on anxiety and depression. The behavioural principles behind stimulus control for insomnia have also been applied to the treatment of pathological worry, the focus of the present dissertation.

\section{Stimulus Control Model of Worry Postponement}

Borkovec, Wilkinson and colleagues (1983) adapted stimulus control for application to worry with the rationale that worry is an "internal, voluntary, operant" behaviour that is under poor stimulus control. They proposed that worry is a response, triggered by threat-relevant stimuli, or what have been labeled "fear cues" (Borkovec, Wilkinson, et al., 1983). In the case of GAD when anxiety or fear is triggered by a perceived threat, individuals engage in worry as a cognitive response to that threat. For individuals with GAD, when worry is triggered it occurs in a persistent and uncontrollable way. The uncontrollability of worry means that worry occurs in a broad variety of situations and thus worry becomes associated with a wide variety of internal and external stimuli that may be unrelated to the original fear cue (Borkovec, Wilkinson, et al., 1983). In this way, worry becomes generalized. For example, a fear about not performing well at work could lead to worry being triggered by any stimuli related to work, such as entering the office, receiving a work email, etc. Worry could also become associated with internal stimuli including certain types of thoughts (e.g., thinking about a work deadline) or physiological changes (e.g., increased heart rate, muscle tension). Generalized worry is proposed to be 
negatively reinforced when a feared negative outcome does not occur (Borkovec et al., 1998). Worry is also proposed to be negatively reinforcing because it dampens emotional arousal. Worry is conceptualized as an "avoidance behaviour" because it takes an abstract form that prevents people from thinking about their fears in a concrete, emotionally arousing way (Borkovec et al., 1998; Stöber \& Borkovec, 2002). As such, worry is proposed to dampen physiological arousal associated with fear cues, and is thus negatively reinforcing. Taken together, uncontrollable worry becomes associated with many different stimuli and these associations are negatively reinforced when the feared outcome does not happen or when emotional arousal is reduced. Based on this conceptualization of pathological worry, the goal of stimulus control for worry is to limit stimulus generalization by reducing the internal and external stimuli that trigger worry. The original instructions for worry postponement are as follows:

(a) Learn to identify worrisome thoughts and other thoughts that are unnecessary or unpleasant. Distinguish these from necessary or pleasant thoughts related to the present moment, (b) Establish a half hour worry period to take place at the same time and in the same location each day, (c) When you catch yourself worrying, postpone the worry to the worry period and replace it with attending to present-moment experience, (d) Make use of the half hour worry period to worry about your concerns and to engage in problemsolving to eliminate those concerns (Borkovec, Wilkinson, et al., 1983, pp. 247-248) Several current recommendations for use of worry postponement closely follow these original instructions (Borkovec, 2006; Newman \& Borkovec, 2002; Rygh \& Sanderson, 2004).

In addition to stimulus control, a goal of worry postponement is to replace the worry response with active coping and problem solving (Borkovec, Wilkinson, et al., 1983). Worry is 
described as an ineffective problem solving strategy (Szabó \& Lovibond, 2006). Worry content in GAD typically involves repeatedly generating possible future negative outcomes and thinking about problems in an abstract way (Borkovec, Robinson, et al., 1983; Stöber, 1998). Catastrophic worrying does not typically include active coping or generating concrete problem solutions and thus concerns about negative outcomes are maintained (Davey, 1994; Stöber, 1998; Szabó \& Lovibond, 2006). The third and fourth instructions ("c" and "d" above) for worry postponement are intended to reduce periods of unproductive worry through attending to the present moment and increasing active problem solving related to one's current concerns by using the worry period to problem solve (Borkovec, Wilkinson, et al., 1983).

\section{Metacognitive Model of Worry Postponement}

Worry postponement is also recommended in metacognitive therapy for GAD under a different rationale than that provided by Borkovec, Wilkinson and colleagues (1983).

Metacognitive theory of GAD proposes that individuals with GAD have positive beliefs about worry (e.g., worry helps me problem solve) and negative beliefs about worry (e.g., worry is uncontrollable and dangerous; Wells, 1999). Negative beliefs about worry are proposed to lead to efforts to avoid triggers for worry and to "metaworry," or worry about worry (Wells, 1999). Worry postponement is used in metacognitive therapy for GAD as a behavioural experiment to test the belief that worry is uncontrollable (Wells, 2006). Because individuals with GAD believe that worry is uncontrollable, they may not engage in efforts to interrupt worry once it has begun (Wells, 1999). By practicing worry postponement individuals with GAD will gain evidence that they can control worry once it has begun. The goal of worry postponement in metacognitive therapy for GAD is to reduce the belief "worry is uncontrollable" and to disrupt the cycle of worry (Wells, 2006). Worry postponement is intended for use in combination with other 
treatment components within a larger metacognitive CBT treatment. Instructions for worry postponement in metacognitive therapy resemble those by Borkovec et al. (1983) with some noteworthy differences. Consistent with the behavioural experiment approach, the instructions do not specify a length of time for the worry period but give the guideline of 15 minutes (Wells, 2006). Additionally the instructions state that the worry period is not compulsory and they may decide not to use it. As with the original instructions, the metacognitive instructions speak to the importance of disengaging from worry rather than efforts to suppress worry. Clients are encouraged to adopt a mindset of "detached mindfulness" (Wells, 2006). This is illustrated in the following instructions:

It is important that you don't confuse postponing your worry with the act of trying not to think a thought. I'm asking you to allow an initial thought that triggers worry to remain in your mind but you are choosing not to engage with it by worrying and trying to work it out. (Wells, 2006, p. 266)

The therapist records their client's percentage belief in the uncontrollability of worry before and after this behavioural experiment, and the experiment is repeated or adjusted as needed until the client's belief reaches zero (Wells, 2006). As such there are not strict instructions for the duration of the intervention.

Stimulus control and metacognitive theory represent the two most common rationales for the use of worry postponement in the treatment of GAD. Very little research has been conducted on the rationales for worry postponement. It is useful to consider other examples of behavioural interventions for repetitive cognition in order to gain a broader theoretical understanding of why worry postponement may be a useful approach to treating worry. 


\section{Other Approaches to Treating Verbal Behaviour in Psychopathology}

Repetitive negative thinking such as worry, occurs across different forms of psychopathology. One notable example is rumination in depression. Depressive rumination and worry have common features in that they both occur repetitively and contain negative, abstract, verbal content (Watkins, 2008). In behavioural activation interventions for depression, behavioural strategies have been applied to reduce rumination. Early behavioural activation treatment for depression involved using "thought stopping" techniques to deal with negative thoughts (Lewinsohn, Biglan, \& Zeiss, 1976). In its modern form, behavioural activation for depression treats rumination as a "maladaptive avoidance behaviour" which is negatively reinforced by short-term relief from negative emotions (Martell, Addis, \& Jacobson, 2001). Clients are instructed to become aware of contexts in which rumination occurs and when they notice rumination to stop the rumination and engage in "alternate coping behaviour" which could include attending to the present moment or problem solving (Martell et al., 2001). The procedures for dealing with rumination in behavioural activation closely resemble worry postponement strategies. Both interventions involve the contingent disruption of repetitive negative thinking in that they involve 1 . Noticing when periods of repetitive negating thinking occur and then 2. Doing something different (i.e., engaging in alternative coping in behavioural activation or putting off worry to a later time in worry postponement). The rumination component of behavioural activation treatment has not been studied in isolation and therefore there is not currently empirical evidence that this approach to rumination is efficacious. The use of behavioural strategies to reduce rumination in depression suggests that parallel strategies may be effective when applied to worry. Further, it suggests that worry postponement may also have 
effects on depressive symptoms and rumination. The effects of worry postponement on other forms of repetitive negative thought, such as rumination, are not known.

\section{Evidence for Worry Postponement}

Two studies have examined the use of worry postponement as an intervention for high trait worry. Borkovec et al. (1983) presented the first evidence of the effects of worry postponement in two experiments. In the first experiment, 4 weeks of daily worry postponement was compared to an assessment only control condition in a sample of students reporting high levels of worry, operationalized as worrying more than half the day and identifying worry as a problem. All participants filled out daily worry questionnaires for 1 week prior to randomization to obtain a baseline measure of worry. Participants assigned to the worry postponement condition met in a group for 1 hour with a therapist who delivered the worry postponement instructions and discussed how to attend to the present moment (Borkovec, Wilkinson, et al., 1983). Participants then independently carried out the worry postponement intervention daily over 4 weeks and also recorded their level of worry on each day of practice. Participants assigned to the assessment only control condition recorded their level of worry on each day for 4 weeks, but did not engage in worry postponement. Change scores from pretreatment to the fourth week of treatment were compared between groups. The worry postponement condition, compared to control, showed a greater a decrease in percent of the day spent worrying (between-group $d=0.84$ ) and amount of focus on "unrealistic concerns" (rated on a 5-point scale; between-group $d=0.63$ ). Visual inspection of group means shows almost no change on worry measures in the control condition. In the control condition, mean percent of the day spent worrying was $41.72 \%$ at pretreatment and $40.08 \%$ at the end of treatment, whereas the worry postponement condition went from $40.31 \%$ to 24.82\% (standard deviations unavailable; Borkovec, Wilkinson, et al., 1983). The second 
experiment compared two forms of worry postponement: one in which participants were instructed to write out their worries during their worry period and the other where they were instructed to mentally worry during the worry period. The authors included a written worry condition based on the rationale that writing about worries could improve focus on worries during the worry period and consequently lead to a greater reduction in worry than mentally worrying (Borkovec, Wilkinson, et al., 1983). The instruction to use the worry period as a time to problem solve was excluded from the worry postponement procedures in the second study. The two worry postponement conditions were compared to a wait list control condition. Procedures were the same as for the first study except that participants were called once per week to check if they had questions about the procedures. Change scores were compared between groups. There were no significant differences between the written and mental worry conditions (effect size unavailable) but both worry postponement conditions, compared to control, led to significant decreases from pretreatment to week four in time spent worrying (effect between control condition and treatment conditions; $d=0.73$ ) and in self-reported tension $(d=0.71)$. The authors, in comparing the findings to those of the first study, concluded that there were no differences based on the inclusion or exclusion of the problem solving instruction. Taken together these studies suggest that worry postponement reduces worry compared to a control condition and that there are no significant differences when instructions are varied to include problem solving or written worry. Whereas these findings are promising, a nonclinical study sample was tested and researchers did not assess participant adherence to intervention instructions. Therefore, the extent to which these findings generalize to clinical populations and the degree to which people actually engage consistently in worry postponement are to be determined. 
It is noteworthy that no studies were published on worry postponement for high trait worry or GAD between 1983 and 2013. The reason for this is not known. Most recently, worry postponement was studied in a sample of individuals with high levels of trait worry (McGowan \& Behar, 2013). A 2-week intervention of daily worry postponement as per Borkovec et al., (1983) was compared to an active control condition. In the active control condition, which was included to account for expectancy effects, participants were instructed to focus on their worry when it occurred (labeled "focused worry"). The focused worry condition was intended to lead to participants engaging in worry as they normally would while creating expectancy for change by providing them with a treatment rationale. Focused worry participants were told that fighting against worry might increase it, so that they should allow worry to naturally occur and to focus their attention on it when it did. There was a high level of compliance to daily worry measures and participants in the worry postponement condition reported completing the worry period $87.5 \%$ of the time across the 2 weeks of the intervention. Compared to the focused worry control condition, after 2 weeks of the intervention, the worry postponement condition showed significantly lower worry (between-group effect size: $d=0.72$ ), anxiety $(d=0.71)$, negative affect $(d=0.55)$, and symptoms of insomnia $(d=0.51)$. Within-group changes from pre to posttreatment were greater in the worry postponement condition than control on all outcome measures including worry (WP; $d=1.81, \mathrm{C} ; d=0.79$ ), anxiety (WP; $d=1.46, \mathrm{C} ; d=0.19$ ), negative affect (WP; $d=1.81, \mathrm{C} ; d=0.49$ ), and insomnia symptoms (WP; $d=0.93, \mathrm{C} ; d=0.02$ ). It is notable that the focused worry condition, intended as a control, lead to a large reduction in worry. The authors speculate that the instructions for the focused worry condition may reduce experiential avoidance by asking participants to actively engage in their worry, and that this mechanism may explain the improvement in worry seen in their control condition (McGowan \& 
Behar, 2013). A mixed analysis of variance (ANOVA) found no Condition x Time effect on depressive symptoms measured using the Beck Depression Inventory (BDI-II). However, the worry postponement condition displayed a large pre to posttreatment change in depressive symptoms $(d=1.10$, Control; $d=0.24)$, with scores dropping from the "moderate" to "minimal" range of clinical severity (Beck, Steer, \& Brown, 1996). There was also a medium betweengroup difference in depressive symptoms at posttreatment; with worry postponement resulting in lower BDI-II scores $(d=0.62)$. There was no significant effect of worry postponement on positive affect (within-group $d=0.41$ ). Clinically significant change was assessed as the proportion of participants in each condition whose scores on posttreatment measures were at least two standard deviations below the pretreatment group mean. In the worry postponement condition $65.2 \%$ of participants evidenced clinically significant change in worry (compared to $30.4 \%$ in the control condition; McGowan \& Behar, 2013). This study is the most rigorous investigation of worry postponement to date and, along with the study by Borkovec, Wilkinson and colleagues (1983), provides promising evidence that this intervention can be helpful at improving a range of symptoms for individuals with pathological worry. The implications of the McGowan and Behar (2013) study are limited by the use of a nonclinical sample. Further, outcomes were measured immediately following a 14-day intervention period, and as such the study does not provide any information on whether results were maintained.

\section{Other Applications of Worry Postponement}

Worry postponement has also been studied in unselected samples. One study (Brosschot \& Van Der Doef, 2006) examined the effects of worry postponement on worry and somatic complaints in high school students. Individuals with GAD experience a high level of health complaints and this contributes to the global burden related to the disorder (Brosschot et al., 
2006). Interventions that reduce worry in GAD may have the additional effect of reducing somatic complaints related to GAD, and health complaints more generally. In an unselected sample, high school students completed 6 days of worry diaries, recording the duration and frequency of their worry. Half were instructed to postpone worry and the other half to just complete worry diaries (control condition; Brosschot \& Van Der Doef, 2006). Participants completed questionnaires at the start of the 6 days and immediately following the sixth day. Compared to the control condition, the worry postponement condition showed significantly shorter average worry duration across the 6 days of the study (between-group difference at postassessment, $d=0.38)$ and significantly fewer health complaints posttreatment $(d=0.44)$. Both conditions demonstrated a significant reduction in health complaints from pre to posttreatment but this effect was larger in the worry postponement condition $(d=0.78)$ than in the control condition (0.40).Worry duration and frequency scores were averaged across the 6 days of the intervention and therefore information on within-group changes in worry duration and frequency is unavailable.

In another study, an unselected sample of school-aged children between 9 and 13 years were assigned to either monitor their negative perseverative thoughts for a week or to monitor and postpone these thoughts to a 30-minute worry period (Jellesma, Verkuil, \& Brosschot, 2009). Perseverative thought duration and frequency was assessed through daily diaries completed over the week of the intervention. Worry duration and frequency was averaged across the 7 days of the study. The authors reported group means separated by gender. Compared to the monitoring condition, participants in the postponement condition displayed a lower average frequency of perseverative thoughts (between-group effect in boys; $d=0.25$, in girls; $d=0.39$ ). There was also a significant effect of condition on worry duration, but this was qualified by a 
significant interaction with gender where girls in the worry postponement condition showed lower worry duration than controls, but boys did not (between-group effect in boys; $d=0.01$, in girls; $d=0.59$ ). Information on within-group changes in worry frequency and duration is unavailable. These studies demonstrate that worry postponement is a simple enough intervention that it can be applied by children and adolescents, that it is effective even when the intervention is brief, and that it can have effects on worry-related symptoms such as health complaints.

One study examined the effects of worry postponement administered as an online intervention with an unselected sample. Versluis, Verkuil, and Brosschot (2016) randomly assigned volunteers to 6 days of worry monitoring or 6 days of worry postponement. Results showed that worry frequency reduced over the 6-day period, but that this did not vary by condition. Further, worry duration did not change significantly in either group. Versluis and colleagues (2016) identified factors that may have contributed to their finding that worry postponement did not lead to greater worry reduction than worry monitoring, including the brief intervention period, participants reporting difficulty postponing worry, and a need to better adapt the intervention for online application.

Although worry postponement has never been studied in a sample of individuals with GAD, it has been studied in other clinical samples. For example, worry postponement was studied as a "pretreatment" in a sample of adults referred for work stress group treatment based on the rationale that reducing perseverative thought would lead to better treatment outcomes (Verkuil, Brosschot, Korrelboom, Reul-Verlaan, \& Thayer, 2011). Participants had diagnoses of adjustment disorder, unspecified somatoform disorder, or were experiencing "severe work problems" (Verkuil et al., 2011). Two weeks of worry postponement prior to the group was compared to monitoring worries alone and to treatment as usual (i.e., no pretreatment before the 
work stress group). After 2 weeks of pretreatment, participants in the worry postponement condition showed a significant decrease in somatic symptoms, but no significant change in anxiety or depressive symptoms (effect sizes unavailable). Immediately after treatment individuals in the worry postponement condition showed fewer anxiety symptoms than did participants in treatment as usual (between-groups $d=0.59$ ). At a 3-month follow-up after completion of group treatment, individuals in the worry postponement condition compared to treatment as usual reported fewer somatic $(d=0.51)$ and depressive symptoms $(d=0.51)$. There were no significant differences between worry postponement and monitoring alone at either time point. The study by Verkuil and colleagues (2011) demonstrates that worry postponement may be a useful adjunct to other treatments and that there may be some therapeutic effects of monitoring worry. One major limitation of this study is that it did not assess changes in worry as a result of treatment, which is the main target of a worry postponement intervention.

Worry postponement has also been studied as a component of a brief worry intervention for people with delusions (Foster, Startup, Potts, \& Freeman, 2010), and as part of a treatment for anxiety in older adults (Wetherell, Sorrell, Thorp, \& Patterson, 2005). Worry postponement is proposed to require limited use of "cognitive linguistic resources" (Wells, 2006), and therefore it is fitting that it has been applied to populations in which these resources may be restricted. Further, it has been suggested that worry postponement places a lower demand on cognitive resources compared to more cognitively intensive interventions like cognitive restructuring (Wells, 2006). As worry has been shown to be cognitively demanding (Hayes, Hirsch, \& Mathews, 2008; Leigh \& Hirsch, 2011; Stefanopoulou, Hirsch, Hayes, Adlam, \& Coker, 2014) worry postponement may be a more appropriate first line intervention for individuals with GAD than more cognitively involved interventions. 
In sum, there are several controlled studies demonstrating that a brief worry postponement intervention can lead to moderate to large improvements in worry and associated symptoms. Despite these promising findings there are several limitations to the existing literature on worry postponement. First, worry postponement contains multiple instructions, the first of which is to monitor when worry occurs. There have been contradictory findings with regard to worry postponement's efficacy relative to merely monitoring worry. Some studies have demonstrated a superiority of worry postponement to worry monitoring (Jellesma et al., 2009), whereas others have found no significant differences (Verkuil et al., 2011). McGowan and Behar's (2013) study included a "focused worry" condition that is similar to a worry monitoring condition - participants were instructed to monitor their worry and when it occurred to focus their attention on it. Individuals in the focused worry condition demonstrated a large reduction in worry from pre to postintervention $(d=0.79)$. It is possible therefore that worry monitoring alone can lead to large changes in worry. However, in the McGowan and Behar study it is unclear whether the large effect is due to monitoring, the instruction to focus on worry, or a combination of the two. It is also important to note that individuals in the worry postponement condition had a significantly larger within-group reduction in worry $(d=1.81)$. Taken together, it is unclear whether there is incremental benefit added by the instruction to disengage and postpone worry or if participants would benefit equally from just monitoring worry.

Second, with one exception (Verkuil et al., 2011), studies on worry postponement have not included a follow-up assessment of outcomes beyond the postintervention assessment; therefore, the durability of demonstrated effects, even in the short-term, is unknown.

Third, worry postponement has never been studied in a sample of people with GAD, the population for whom it is recommended most explicitly. It is unknown whether the effects 
demonstrated in existing studies would generalize to a sample reporting clinically significant levels of worry.

Fourth, the effects of a worry postponement intervention on worry-related cognitive processes have not been investigated despite their importance to the psychopathology of GAD. Worry is associated with a host of maladaptive cognitive processes such as cognitive avoidance (Borkovec, Alcaine, \& Behar, 2004), negative problem orientation (Dugas, Letarte, Rhéaume, Freeston, \& Ladouceur, 1995; Dugas, Gagnon, Ladouceur, \& Freeston, 1998), and difficulty with attentional control (Hirsch \& Mathews, 2012), which contribute to the impairment that people with GAD experience and serve to maintain the disorder. An effective worry intervention should also lead to changes in worry-related cognitive processes.

Finally, although there are multiple proposed rationales for the use of worry postponement, the specific effects of worry postponement on pathological worry are unclear and the mechanisms of change associated with this intervention have not been empirically studied.

\section{Effects of Worry Postponement on Pathological Worry}

Pathological worry has several features; it is excessive in frequency and duration, and is perceived by the individual as uncontrollable. It is unknown which of these features are most affected by worry postponement. Borkovec, Wilkinson and colleagues (1983) have proposed that worry postponement reduces pathological worry by bringing worry under stimulus control. If stimulus control theory of worry postponement is accurate, one would expect that over the course of the intervention, the frequency of worry episodes should decrease. If worry postponement leads to greater stimulus control over worry, over time worry should come to be associated with the worry period and the associations between worry and other stimuli should weaken. If a smaller range of stimuli elicits worry (i.e., worry is under stimulus control) then one 
would expect to see fewer occurrences of worry throughout the day. Worry frequency is just one possible index of stimulus control. Assessing worry frequency has benefits over assessing other indices of stimulus control as it is relatively easy to measure and compare across participants. Two studies, both in healthy children and adolescents, examined changes in frequency of worry. One study found that worry postponement relative to worry monitoring led to a significant decrease in worry frequency as measured through daily diary averages across treatment (between-group effect in boys; $d=0.25$, in girls; $d=0.38$; Jellesma et al, 2009), whereas the other study found no significant differences between these conditions in worry frequency following a 1-week intervention (between-group $d=0.24$; Brosschot $\&$ Van Der Doef, 2006). Both studies that reported on changes in worry frequency had brief, week-long interventions, and averaged frequency across all days of the study. Both of these methodological factors could have led to the underestimating of the effect of worry postponement on worry frequency. Based on the literature, therefore, it is currently unknown whether worry postponement in people with GAD leads to stimulus control of worry.

\section{Proposed Mechanisms of Change in Worry Postponement}

Change in metacognitive beliefs is one of the primary mechanisms that has been proposed to explain worry postponement's effects on pathological worry. From the perspective of the metacognitive theory of worry postponement, one would expect that the belief or perception that worry is uncontrollable would decrease over the course of the worry postponement intervention, leading to changes in worry and associated symptoms.

Another possible explanation for the effects of worry postponement on worry and related symptoms is that the intervention trains individuals to disengage from their worry, increasing their attentional control over negative repetitive thinking. Worry postponement requires 
individuals to actively practice disengaging from worry repeatedly throughout the day when they experience negative thought intrusions. According to metacognitive theory, individuals with GAD do not typically make efforts to disengage from worry once it starts (Wells, 1999). By training individuals to notice and disengage from worry, worry postponement may increase attentional control, especially attentional control over negative thoughts.

Attentional control refers to the ability to maintain goal-focused attention, to inhibit irrelevant information from attention, and to shift attention between different tasks (Eysenck, Derakshan, Santos, \& Calvo, 2007; Miyake et al., 2000). Attentional control maps onto the central executive component of working memory (Miyake et al., 2000). One feature of GAD is difficulty concentrating (American Psychiatric Association, 2013). Attention has been implicated as an important factor in the development and maintenance of perseverative negative thought (Hirsch \& Mathews, 2012; Matthews \& Wells, 2000). Difficulty concentrating could be due to worry's negative effects on attentional control processes. In their cognitive model of pathological worry, Hirsch and Mathews (2012) propose that worry is maintained, in part, by impaired attentional control which causes difficulty disengaging from worry. They further argue that sustained worry may lead to increased attention to threat and further negative thought intrusions, thus maintaining anxiety and worry. There is evidence that higher trait worry is related to poorer performance on attentional control tasks (Crowe, Matthews, \& Walkenhorst, 2007) and that worry is a cognitively demanding mental activity relative to positive or neutral forms of thinking (Hallion, Ruscio, \& Jha, 2014; Hayes et al., 2008; Leigh \& Hirsch, 2011; Stefanopoulou et al., 2014). The cognitive demands imposed by worry may lead to concentration difficulties and to difficulty exerting attentional control to inhibit episodes of worry and disengage from worry once it has begun. Worry postponement may increase attentional control over worry, and 
attenuate the negative effect of worry on attentional processes by training individuals to notice worry and redirect their attention to the present moment. Hirsch and Mathews (2012) identified worry postponement as an intervention that attempts to train attentional control over worry.

Increased attentional control, especially attentional control over worry, may be a mediator of the effects of worry postponement on worry that is not captured in the stimulus control and metacognitive rationales for the intervention.

\section{Proposed Effects of Worry Postponement on Symptoms and Cognitive-Processes Related to Generalized Anxiety Disorder}

Worry postponement may have effects on symptoms and cognitive processes related to worry in GAD. In individuals high in the tendency to worry, there is initial evidence that worry postponement has positive effects on symptoms of insomnia (McGowan \& Behar, 2013), health complaints (e.g., pain and headache, gastrointestinal problems, cold and flu symptoms; Brosschot \& Van Der Doef, 2006), and negative affect (McGowan \& Behar, 2013). McGowan and Behar (2013) additionally found a medium between-group effect size for worry postponement's effect on depressive symptoms though this finding did not reach significance. In my review of the literature on worry postponement, I noted that not a single study has examined an outcome other than symptoms. There are theoretical reasons to hypothesize that worry postponement may lead to improvement in cognitive processes related to GAD including mindfulness, problem solving quality, and cognitive avoidance. These outcomes have not, to my knowledge, been investigated in past studies.

Problem Solving. Worry postponement may have positive effects on individuals' attitudes toward problems and the process of solving them. Individuals with GAD have a “negative problem orientation" - a tendency to see problems as threatening, to doubt one's own 
problem solving ability, and to be pessimistic about a problem's outcome (Gosselin, Pelletier, \& Ladouceur, 2001). Excessive, catastrophic worry is, at the core, a misguided, and typically unproductive attempt to solve a problem (Borkovec, Wilkinson, et al., 1983). According to the intolerance of uncertainty model of GAD, people with pathological worry have negative beliefs about problems and the problem solving process. When people engage in worry in response to a problem that is unsolvable (e.g., it may not even exist), this may lead to frustration and anxiety regarding problems, and foster beliefs that one cannot effectively solve problems and that problems are insurmountable (Dugas et al., 1995; Dugas et al., 1998). When people with GAD confront real problems they tend to view them as threatening and may get mired in gathering information about the problem rather than proceeding through the problem solving process (Dugas et al., 1998; Ladouceur, Talbot, \& Dugas, 1997). In current treatments for GAD, a more positive problem orientation is trained by helping participants to distinguish between worries that are amenable to problem solving and those that are not, to identify key elements of problems, and to take actions to solve problems. I propose that worry postponement may be also be a strategy that reduces a negative problem orientation, in that individuals are instructed to actively and purposefully turn toward their worries and engage in problem solving (or disengage from worry if the problem does not exist at the moment or is unsolvable). By approaching their problems in this way, people may come to see their problems as less threatening and more manageable, thus adopting a less negative problem orientation. Further, worry postponement may have effects on problem solving style. Worry postponement encourages a more active problem solving style by encouraging people to approach and think through the problem solving process. Further, worry postponement could decrease impulsive problem solving; by delaying 
worry, people may find that they can tolerate problems without needing to respond to them immediately.

Mindfulness. Worry postponement may have effects on mindfulness, defined as selfregulated attention to the present moment and an orientation of openness, curiosity, and acceptance (Bishop et al., 2004). The original guidelines for worry postponement instruct the person to attend to "present moment experience" (Borkovec, Wilkinson, et al., 1983). When worry postponement is practiced within the context of metacognitive therapy, people are instructed to adopt a stance of "detached mindfulness." The instructions suggest that worry postponement may increase mindfulness. Worry has been conceptualized as a form of "experiential avoidance" meaning that it is used by people to avoid and withdraw from uncomfortable or anxiety provoking experiences (Roemer \& Orsillo, 2002). In mindfulnessbased therapies for GAD, mindfulness strategies are used to counter the tendency toward worry and experiential avoidance. These therapies involve a variety of strategies including mindfulness meditation, decentering from negative thoughts, and practicing acceptance of negative experiences. There is initial evidence that mindfulness-based therapies are efficacious at reducing worry and GAD symptoms (Craigie, Rees, Marsh, \& Nathan, 2008; Evans et al., 2008; Orsillo, Roemer, \& Barlow, 2003; Roemer \& Orsillo, 2007; Roemer, Orsillo, \& SaltersPedneault, 2008). Strategies used in worry postponement share similarities with those used in mindfulness-based treatments. For example, in postponing worry individuals disengage from negative thoughts and attend to present moment experience, which is the same skill that is practiced in mindfulness meditation. In worry postponement, during the worry period people are encouraged to approach anxiety-provoking topics they may have been avoiding thinking about deeply. The worry period therefore may be a strategy for increasing mindfulness by countering 
the experiential avoidance that is thought to be central to GAD (Roemer \& Orsillo, 2002). As such, as well as reducing worry, worry postponement may have effects on processes similar to those targeted in mindfulness and acceptance based therapies. The present study considered multiple facets of mindfulness including mindful attention, acceptance of internal experiences, and mindful reactions to unwanted thoughts and images.

Cognitive Avoidance. Worry postponement may reduce cognitive avoidance. Worry is proposed to facilitate cognitive avoidance of mental imagery, specifically, thinking about feared situations in a concrete, vivid, and emotionally arousing way (Borkovec et al., 1998; Stöber, 1998). The cognitive avoidance theory of worry proposes that when individuals with GAD are confronted with fear-arousing stimuli, they use cognitive strategies such as worry to dampen the emotional arousal associated with fear related mental imagery (Borkovec et al., 1998). The reduced concreteness theory of worry further suggests that worry's abstract content allows individuals to avoid thinking about feared stimuli in a concrete, emotionally arousing manner (Stöber, 1998). It has been suggested that cognitive avoidance, especially avoidance of mental images of frightening worst case scenarios, may be a central process in pathological worry (Dugas et al., 1998). Cognitive avoidance is also proposed to interfere with the emotional processing of anxiety-provoking material, and consequently, to maintain anxiety and worry (Borkovec et al., 1998). Worry postponement encourages people to mentally approach topics they are afraid of during the designated worry period and encourages them to actively problem solve (or disengage from) issues they have been worrying about. Problem solving requires individuals to think about issues in a clear and concrete manner inconsistent with cognitive avoidance. Further, in the worry period, thinking about hypothetical worries that may not be amendable to problem solving could serve as a form of "cognitive exposure" which has been 
proposed to be helpful for reducing cognitive avoidance and worry (Dugas et al., 1998). The active approach to concerns encouraged in worry postponement may reduce cognitive avoidance by providing people with another strategy for dealing with upsetting mental experiences and facilitating emotional processing. Use of worry postponement may reduce reliance on cognitive avoidance strategies such as suppression, worry, or avoiding stimuli that may trigger thoughts and images about feared situations.

\section{The Present Study}

The present study examined the immediate and short-term effects of a 2-week daily worry postponement intervention in people with GAD. Worry postponement (WP) was compared to an active control condition consisting of 2 weeks of worry monitoring alone (MON), and to an assessment only control condition (AX-ONLY). WP contains multiple components including monitoring the occurrence of worry, disengaging from worry, and engaging in a worry period. The MON condition was included as an active control to account for the effects of monitoring episodes of worry as it is yet unknown whether postponing worry has an effect over and above merely monitoring when worry occurs. The most rigorous WP study to date (McGowan \& Behar, 2013) included an active control condition labeled "focused worry." The focused worry condition combined worry monitoring with the instruction to focus on worry when it occurs. As such, the McGowan and Behar (2013) study did not allow for conclusions to be made about the effects of monitoring alone relative to monitoring and WP. This is an important outstanding question because worry monitoring constitutes a large part of the WP intervention and one study showed that it has comparable effects to WP (Verkuil et al., 2011). The inclusion of a worry monitoring condition in the present study allowed for clear conclusions to be made about the effects of worry monitoring relative to the effects of WP. In the AX-ONLY 
condition, participants completed baseline and follow-up assessments but did not complete daily worry monitoring. The AX-ONLY control was included to control for the effects of the passage of time and the effects of repeatedly filling out questionnaire measures on worry and related symptoms. Participants in the active conditions (WP and MON) completed 2 weeks of daily worry monitoring (MON) or worry monitoring plus worry postponement (WP). All participants were assessed at baseline, immediately following the 2-week intervention, and 2 weeks after the end of the intervention period. The present study aimed to address questions about the efficacy of WP for reducing worry in GAD, the broader effects of WP on symptoms and cognitive processes related to GAD, and mechanisms through which WP has its effects.

\section{Research Questions and Hypotheses}

Question 1: Does WP lead to statistically and clinically significant change in worry and other GAD symptoms in a sample of people with GAD?

Hypothesis 1: Regarding the effects of WP on worry and GAD symptoms, it was predicted that:

A) Within the WP condition there would be statistically significant reductions in worry and GAD symptoms which would be evidenced at postintervention and at a 2-week follow-up. Further, it was predicted that the MON condition would lead to reduction in worry and GAD symptoms but that the magnitude of this reduction would be less than in the WP condition. It was not expected that there would be a significant reduction in worry or GAD symptoms in the AXONLY condition. It was predicted that WP would lead to statistically significantly lower scores on worry and GAD symptom measures compared to monitoring alone and an assessment-only control condition and this would be seen at postintervention and 2-week follow-up. 
B) A greater proportion of individuals in the WP condition than in the other conditions would demonstrate a clinically significant change in worry and GAD symptoms. Clinically significant change was operationalized as the proportion of individuals whose postintervention scores on measures of past-week pathological worry or GAD symptom severity fell at least two standard deviations below their preintervention score (Jacobson, Follette, \& Ravenstorf, 1984).

Question 2: Does WP lead to statistically significant improvements in cognitive processes related to GAD, specifically: mindfulness, problem solving, cognitive avoidance, and attentional control?

Hypothesis 2: It was predicted that within the WP condition there would be a statistically significant increase in mindfulness and in attentional control, and a statistically significant decrease in negative problem orientation and cognitive avoidance. As this is a new area of investigation, no predictions were advanced as to whether the MON condition would demonstrate changes in these processes. Changes in cognitive processes were not expected to be seen within the AX-ONLY condition. It was further predicted that there would be statistically significant differences between the three conditions. That is, WP would lead to significantly greater increases in mindfulness, and attentional control and significantly greater decreases in negative problem orientation, and cognitive avoidance compared to monitoring alone and an AX-ONLY control condition.

Question 3: Does WP lead to greater stimulus control over worry?

Hypothesis 3: Change in worry frequency was examined as an indication of worry coming under stimulus control. Worry frequency was operationalized as the number of distinct worry episodes participants reported in a worry diary within a 24-hour period. It was predicted 
that if WP works through stimulus control as is theorized, one would expect to see a significant reduction in worry frequency across the 2 weeks of the intervention.

Question 4: What are the mechanisms of WP's effects on worry reduction?

Hypothesis 4: Two potential mediators of WP's worry-reducing effects were identified a priori: metacognitive shift operationalized as a decrease in the self-reported belief that worry is uncontrollable, and ability to disengage from worry operationalized as an improvement in attentional control over worry. It was predicted that both metacognitive shift and changes attentional control would mediate changes in worry resulting from WP.

Question 5: Does WP lead to statistically and clinically significant improvements in depressive symptoms, anxiety, symptoms of insomnia, and health complaints in people with GAD?

Hypothesis 5: It was predicted that within the WP condition, individuals would show a statistically significant reduction in depressive symptoms, anxiety, symptoms of insomnia, and health complaints at postintervention and at the 2-week follow-up. Previous studies have found that monitoring worry alone leads to small effects on these symptoms ( $d$ s ranging from 0.02 to 0.40; Brosschot \& Van Der Doef, 2006; McGowan \& Behar, 2013). Based on this, it was predicted that the MON condition would show reduction in these symptoms but that the magnitude of this reduction would be small and less than in the WP condition. It was not expected that there would be a significant reduction in symptoms in the AX-ONLY condition. It was predicted that there would be statistically significant between-group differences. Compared to those assigned to the control conditions, those assigned to WP would report less severe depressive symptoms, lower anxiety, less severe insomnia symptoms, and fewer health complaints at postintervention assessment and at 2-week follow-up. A greater proportion of 
individuals in the WP condition than the other conditions would demonstrate a clinically significant change (defined as above) in depressive symptoms, anxiety, symptoms of insomnia, and health complaints. 


\section{Method}

\section{Power Analysis}

An a priori power analysis was conducted using $\mathrm{G}^{*}$ Power (Faul, Erdfelder, Lang, \& Buchner, 2007) to estimate the sample size needed to demonstrate a statistically significant difference between the effects of WP and control conditions on worry. Because there are no standardized procedures to conduct an a priori power analysis for MLM, the power analysis was conducted based on a mixed ANOVA design. As MLM is considered to be a more powerful statistical test than ANOVA (Quené \& van den Bergh, 2004) this approach should provide a conservative sample size estimate. Post hoc power analyses were performed to determine the achieved power in the most recent worry postponement study (McGowan \& Behar, 2013). Also, based on effect sizes found by McGowan and Behar (2013), a priori power analyses were conducted to determine the sample size that would be required to achieve power of 0.80 . McGowan and Behar (2013) found a large between groups effect of worry postponement on worry $(d=0.72)$ and with their sample of 53 participants had an achieved power of 0.90 . In order to detect an effect of the same magnitude, an a prior power analysis determined that a total sample size of 45 would be required. On other outcome measures (negative affect and insomnia symptoms) McGowan \& Behar (2013) found medium effect sizes (between group $d \mathrm{~s}=0.55$ and 0.51 respectively). For these medium effects, McGowan and Behar achieved suboptimal power, 0.62. An a priori power analysis determined that for the present study to detect a medium effect, a sample size of 90, or 30 participants per condition, would be required.

\section{Participants}

Participants with a principal diagnosis of DSM-5 defined GAD were recruited from the community using flyers posted on university and college campuses, online classified 
advertisements (i.e., Craigslist, Kijiji, Facebook), newspaper print advertisements, and a database of individuals who had previously participated in worry-related research in the Cognition and Psychopathology Lab. Potential participants $(N=396)$ were screened over the telephone to assess eligibility criteria. During the phone screen they answered questions about worry and other symptoms of GAD, and were administered the Mini International Neuropsychiatric Interview 7.0.0 (MINI 7.0.0; Sheehan, 2014). A Clinical Severity Rating (CSR) was made to record level of distress and impairment associated with any diagnoses that were present (range 0 to 8 , with 4 indicating clinically significant symptom severity; Brown \& Barlow, 2013).

Participants were included if they: 1) endorsed symptoms consistent with a principal diagnosis of GAD as defined by DSM-5 (American Psychiatric Association, 2013), with a CSR $\geq 4$, and 2) if a comorbid diagnosis was present, its associated CSR was at least 1 point lower than that of their GAD diagnosis. Participants were excluded if they 1) had a current or past history of psychosis or mania, or endorsed symptoms consistent with a diagnosis of a substance use disorder in the past 12 months, 2) reported clinically significant suicidal ideation, intent, or plan, 3) were receiving regular psychotherapy (i.e., a frequency of weekly or more) that had a duration of less than 12 weeks $^{1}, 4$ ) were taking psychotropic medications and had had a change in dose in the past 12 weeks. If they had recently discontinued a psychotropic medication, they were included if it had been at least 1 month since discontinuation, or 3 months if they had been taking fluoxetine. If a participant was taking psychotropic medication on an as needed basis (e.g., benzodiazepines), they were included and their use of this medication was noted. A total of 103 participants were determined to be eligible to participate and were invited to the lab. Of these, 83

\footnotetext{
${ }^{1}$ During the initial stages of recruitment, participants were excluded if they had received psychological treatment or counselling the past 3 months. Due to slow recruitment, this criterion was updated to include a greater number of participants, while still excluding participants who had recently started a new treatment.
} 
participants enrolled in the study, completed the first lab visit, and were randomized to one of the three study conditions (WP; $n=29$, MON; $n=28$, AX-ONLY; $n=26$ ). Following randomization, seven participants in the WP condition and five participants in the MON condition did not attend either the second third lab visits, and/or did not meet the compliance criterion for their condition. Adequate compliance was operationalized as completing the assigned intervention on at least $50 \%$ of intervention days, according to participant self-report. In the AX-ONLY condition, four participants did not attend either the second or third follow-up visits. The final sample for analysis comprised 67 individuals (WP; $n=22$, MON; $n=23$, AXONLY; $n=22$ ) who had completed at least two lab visits, and in the active conditions, demonstrated adequate compliance to the intervention. A summary of participant flow can be found in Appendix A.

Participants in the final sample were compared on demographic and baseline outcome measures to those who were randomized but not included in the final sample due to drop out or noncompliance. Participants included in the final sample endorsed less of a rational problem solving style than did those excluded from analyses, $t(81)=2.00, p<.05, d=0.56$. Further, participants included in the final sample in the WP and MON conditions indicated on the Credibility and Expectancy Questionnaire (CEQ; Borkovec \& Nau, 1972; Devilly \& Borkovec, 2000) that they thought the intervention was more likely to be successful than did those who had been randomized to WP or MON but were not included in analyses, $t(53)=-2.16, p<.05, d=$ 0.67. There were no other significant differences on outcome measures, and no significant differences in demographic factors or assigned condition between those included in the final sample and those excluded. 


\section{Sample Characteristics}

The final sample consisted of 60 females and 7 males, between the ages of 18-57 $(M=$ 29.66, $S D=11.23)$. Participants identified their racial/ethnic background as European origin/White 47.8\%, Asian-American/Asian Origin/Pacific Islander 28.4\%, Middle Eastern 7.5\%, Biracial/Multiracial 4.5\%, African-American/Black/Caribbean Origin 4.5\%, Latinoa/Hispanic 3.0\%, or "Other" $4.5 \%$ (i.e., participants identified with an racial/ethnic background other than those listed). Thirty-two participants indicated that they were students (full-time; $n=$ 25, part-time; $n=7$ ) and 44 reported that they were currently employed (full-time; $n=22$, parttime; $n=22$ ). One-way ANOVA and chi-square tests were used to investigate group differences in demographic characteristics. There was a significant between-group difference in age, $F(2)=$ 3.887, $p<.05$. Posthoc tests indicated that participants in the AX-ONLY condition were significant younger than participants in the MON condition $(p=.007, d=0.83)$. There were no other significant demographic differences between the conditions.

Per inclusion criteria, all participants met DSM-5 criteria for GAD, as assessed by the MINI. Seventeen participants also met criteria for one or more comorbid disorders including major depressive disorder $(n=10)$, social anxiety disorder $(n=7)$, obsessive-compulsive disorder $(n=2)$, bulimia nervosa $(n=1)$, panic disorder $(n=1)$, and agoraphobia $(n=1)$. There was no significant difference in the presence of comorbid disorders across the three conditions, $\chi^{2}(2)=2.39, p=.30$.

Ten participants indicated at baseline that they were currently receiving one or more treatments for a psychological or interpersonal issue (individual counselling; $n=6$, medication; $n$ $=7$ ). No participants reported beginning a new treatment during the first 2 weeks of the study.

Four participants indicated that they began a new treatment for a psychological issue between the 
postintervention visit and follow-up visit (medication; $n=2$, individual counselling; $n=1$, selfhelp manual; $n=1)$. There was no difference between conditions in whether participants were receiving treatment at baseline, $\chi^{2}(2)=.88, p=.64$, or started a new treatment during the course of the study, $\chi^{2}(2)=.40, p=.82$.

See Table 1 for sample characteristics separated by condition.

\section{Materials}

\section{Screening Measures.}

The Mini International Neuropsychiatric Interview 7.0.0 (MINI 7.0.0; Sheehan, 2014) is a semistructured diagnostic interview to assess DSM-5 psychiatric disorders. Information is not yet available on the psychometric properties of the MINI 7.0.0. The MINI 7.0.0 was adapted from the MINI for DSM-IV (Sheehan et al., 1998) which has high diagnostic sensitivity (91\%) and specificity $(86 \%)$ and good test-retest reliability for GAD $(\mathrm{k}=.78$; Sheehan et al., 1997). As diagnostic criteria for GAD have not changed from DSM-IV to DSM-5, the MINI 7.0.0 should provide a reliable and valid assessment of GAD. The MINI was administered over the phone during phone screening procedures to ensure that participants' symptoms met diagnostic criteria for GAD before being invited to participate in the study.

\section{Outcome Measures.}

Internal consistency for self-reported outcome measures is reported in Table 2. All measures had acceptable to excellent internal consistency across the three assessment points of the study. Internal consistency was not calculated for the Generalized Anxiety Disorder Question-IV (GAD-Q-IV), per Newman et al., 2002, as its instructions require participants to discontinue the measure if they score below a certain threshold on early items, leading to invalid reliability estimates. 


\section{Symptom Measures.}

The Penn State Worry Questionnaire (PSWQ; Meyer, Miller, Metzger, \& Borkovec, 1990) is a 16-item self-report measure of the tendency to engage in excessive and uncontrollable worry. The PSWQ has shown good reliability and validity across clinical and nonclinical populations (Brown, Antony, \& Barlow, 1992; Davey, 1993; Meyer et al., 1990) and high internal consistency ( $\alpha=.88$ to .95; Molina $\&$ Borkovec, 1994). The PSWQ was used in this study to describe the sample.

The Penn State Worry Questionnaire - Past Week (PSWQ-PW; Stöber \& Bittencourt, 1998) is an adapted version of the Penn State Worry Questionnaire (Meyer et al., 1990) intended to assess weekly changes in worry. The PSWQ-PW has high reliability and validity and good convergent validity with other measures of weekly worry (Stöber \& Bittencourt, 1998). Given the brief duration of the WP intervention, the PSWQ-PW was used for hypothesis testing. The PSWQ-PW is sensitive to treatment-related changes in worry (Stöber \& Bittencourt, 1998; Woelk \& Schläfke, 2010).

The Generalized Anxiety Disorder Question-IV (GAD-Q-IV; Newman et al., 2002) is a self-report measure of DSM-IV diagnostic criteria for GAD consisting of 9 items. Scores on the measure range from 0 to 13 with a cut score of 7.6 representing $85 \%$ sensitivity and $74 \%$ sensitivity in differentiating between people with and without GAD (Moore, Anderson, Barnes, Haigh, \& Fresco, 2014). The GAD-Q-IV has high convergent validity with other measures of GAD symptoms, good discriminant validity with measures of depression (Robinson, Klenck \& Norton, 2010), and good test-retest reliability (Newman et al., 2002). This measure was used in hypothesis testing to determine the effects of WP on symptoms of GAD. 
The Depression Anxiety Stress Scales, 21-item version (DASS-21; Lovibond \& Lovibond, 1995; Antony et al., 1998) is a 21-item dimensional measure of depression, anxiety, and distress symptoms over the past week. The DASS-21 contains three scales measuring symptoms related to depression, anxiety, and stress. The DASS-21 has good internal consistency and concurrent validity in clinical and community populations (Antony et al., 1998). Only the depression and anxiety subscales were used for hypothesis testing.

The Insomnia Severity Index (ISI; Bastien, Vallières, \& Morin, 2001) is a 7-item selfreport screener for insomnia that assesses perceived sleep difficulties. It was developed for use as an outcome measure in treatment studies (Bastien et al., 2001). The ISI has good internal consistency and reliability and is sensitive to changes in sleep difficulties that occur with treatment. Additionally, the ISI has convergent validity with clinician- and significant other-rated sleep difficulty. The ISI was used in a previous investigation of WP for high trait worry (McGowan \& Behar, 2013).

The Subjective Health Complaints Questionnaire (SHCQ; Eriksen, Ihlebæk, \& Ursin, 1999) is a self-report questionnaire that assesses the severity and duration of 29 health complaints. The SHCQ assesses complaints in five domains: musculoskeletal pain, pseudo neurological symptoms (e.g., dizziness), gastrointestinal problems, allergy, and flu symptoms. The SHCQ can be scored for total number of health complaints as well as complaints in specific domains. The present study used total number of complaints. The original SHCQ assesses the presence of symptoms over the past 4 weeks. Given the timeline of the study and as per Brosschot and Van Der Doef (2006), the questionnaire was revised to assess for symptoms over the past week when administered in the two follow-up sessions. The SHCQ has good internal 
consistency (Eriksen et al., 1999) and good test-retest reliability (Brosschot \& Van Der Doef, 2006).

\section{Process Measures.}

The Cognitive Avoidance Questionnaire (CAQ; Sexton \& Dugas, 2008) is a 25-item measure of the tendency to use five different cognitive avoidance strategies to manage unwanted thoughts. The strategies assessed comprise five subscales: thought suppression, thought substitution, distraction, avoidance of threatening stimuli, and the transformation of images into thoughts. The CAQ has good convergent and divergent validity, and good internal consistency and temporal stability (Sexton \& Dugas, 2002).

The Metacognitions Questionnaire-30 (MCQ-30; Wells \& Cartwright-Hatton, 2004) is a self-report questionnaire with 30 items assessing metacognitive beliefs. The MCQ has five subscales: cognitive confidence, positive beliefs about worry, cognitive self-consciousness, negative beliefs about thoughts concerning uncontrollability and danger, and beliefs about need to control thoughts. The MCQ has good test-retest reliability, convergent validity, and internal consistency (Wells \& Cartwright-Hatton, 2004). The "beliefs about the uncontrollability and dangerousness of thoughts" subscale of the MCQ was used to test the mediation hypothesis (hypothesis 5).

The Attentional Control Scale (ACS; Derryberry \& Reed, 2002) is a 20-item measure of self-reported voluntary attention control. The ACS assesses three related factors: ability to focus attention, ability to shift attention between tasks, and ability to flexibly control thought (Derryberry \& Reed, 2002). The ACS has good internal reliability $(\alpha=.88$; Derryberry \& Reed, 2002). Scores on the ACS are related to the modulation of attentional response when viewing fear-related images (Mathews, Yiend, Lawrence, 2005) and correlate with a behavioural measure 
of attentional control, the Random Interval Generation task ( $r=0.34$; Tallon, Koerner, \& Yang, 2016).

The Cognitive and Affective Mindfulness Scale-Revised (CAMS-R; Feldman, Hayes, Kumar, Greeson, \& Laurenceau, 2007; Hayes \& Feldman, 2004) is a 12-item self-report measure that provides a brief assessment of individual differences in mindfulness. The CAMS-R includes items related to mindful attention (e.g., "it is easy for me to concentrate on what I am doing") as well as items related to acceptance of internal experiences (e.g., "I am able to accept the thoughts and feelings I have"). The CAMS-R has adequate internal consistency, convergent validity with other measures of mindfulness, and divergent validity with measures of emotional regulation and problem solving (Feldman et al., 2007).

The Southampton Mindfulness Questionnaire (SMQ; Chadwick et al., 2008) is a 16-item self-report measure assessing mindful reactions to upsetting thoughts or images. For example, items include "Usually when I experience distressing thoughts and images...I am able just to notice them without reacting" or "In my mind I try to push them away". The SMQ has good internal consistency, convergent validity with other mindfulness measures, and can distinguish between people who meditate and those who do not (Chadwick et al., 2008). Whereas the CAMS-R provides a general assessment of "mindfulness," the SMQ was also included in the present study as it provides an in depth assessment of reactions to unpleasant thoughts and images. This is relevant to WP, as worry is a maladaptive reaction to unwanted thoughts and images, and WP attempts to alter this reaction.

The Negative Problem Orientation Questionnaire (NPOQ; Gosselin, Pelletier, \& Ladouceur, 2001; Robichaud \& Dugas, 2005a) is a 12-item self-report measure of the predisposition to doubt one's own problem solving abilities, to perceive problems as threats, and 
to be pessimistic about the outcome of problems. The NPOQ has good test-retest reliability, high internal consistency $(\alpha=.91)$, and good construct validity (Robichaud \& Dugas, 2005a, 2005b).

The Social Problem Solving Inventory-Revised (SPSI; D'Zurilla, Nezu, \& MaydeuOlivares, 2001) is a self-report measure that assesses problem solving tendencies. The short form of the SPSI-R, used in the present study, has 25 items. The total score on the SPSI reflects global problem solving skills. The SPSI has five subscales: negative problem orientation, positive problem orientation, rational problem solving style, impulsivity/carelessness style, and avoidant style. The SPSI has good test-retest reliability and good internal consistency (D'Zurilla et al., 2001). The Negative Problem Orientation scale was not analyzed as the NPOQ is a more thorough measure of this construct.

The Ruminative Response Scale (RSS; Treynor, Gonzalez, \& Nolen-Hoeksema, 2003) is a 22-item questionnaire assessing the tendency to engage in depressive rumination. The RRS has two scales assessing two forms of rumination; reflection and brooding. The RRS has good internal consistency (Treynor et al., 2003) and acceptable convergent and predictive validity (Nolen-Hoeksema \& Morrow, 1991). The RRS was used in an exploratory manner to examine whether WP influences another form of repetitive negative thinking - rumination.

\section{Performance Measures of Attentional Control.}

The Random Interval Generation Task (RIG; Leigh \& Hirsch, 2011) is a computer-based task assessing working memory capacity. Participants are instructed to press the space bar key on a computer keyboard in a random and unpredictable rhythm, approximately once per second, for 5 minutes. Participants were provided with the task instructions and then given a 15 -second practice trial. Feedback was given if necessary prior to participants completing the 5-minute test trial. Key presses were converted into a series of time intervals and scored to provide two indices 
of randomness: Redundancy ( $R$; Attneave, 1959; Baddeley 1966) and Random Number Generation score (RNG; Evans, 1978). For a description of scoring procedures see Leigh \& Hirsch, 2011. R scores range from 0 to $100 \%$ and RNG scores range from 0 to 1 . For both indices, higher scores reflect less random performance and lower working memory capacity. Performance on the RIG task loads onto the central executive component of working memory which is responsible for controlled attention (Vandierendonck, De Vooght, \& Van der Goten, 1998). This task has been used in previous investigations of working memory in individuals with high levels of trait worry (Leigh \& Hirsch, 2011). This measure was used to test hypothesis 3 , regarding the effects of WP on attentional control.

The N-back task (Owen, McMillan, Laird, \& Bullmore, 2005) is a measure of general attentional control capacity. Participants are presented with a sequence of letters on a computer screen. They are instructed to indicate with a key press when a target letter (e.g., "X") is identical to the letter appearing " $\mathrm{n}$ " positions before. The task is administered in 1-, 2-, and 3-back conditions, where " $n$ " represents the degree of cognitive load. Participants completed three practice trials, one for each n-back condition, with 10 letters in each trial. For the experimental trials, 45 letters were in each trial. Accuracy of responses was recorded. The n-back task has been used previously in a study assessing the effects of worry on attentional control (Stefanopoulou et al., 2014). This measure was included to test hypothesis 3. Two attentional control tasks were used in the present study to obtain a comprehensive assessment of general attentional control. The RIG task is a relatively pure measure of the central executive while the n-back assesses central executive functioning and also taps into attentional storage capacity.

The Breathing Focus Task (Borkovec, Robinson, et al., 1983; Hirsch, Hayes, \& Matthews, 2009; Ruscio \& Borkovec, 2004) assesses attention focusing ability before and after a 
brief period of worry. Participants are instructed to focus their attention on their breathing for a 5-minute period. At 12 points throughout this period, a computer tone cues participants to report on the content of their thought; if it was focused on their breathing, or if focusing on something else, what the valence of the thought was (positive, negative, neutral) and a brief description of the content (e.g., "neutral - lunch"). Following the 5-minute breathing period the researcher prompts the participant to elaborate on what they had been thinking about during each of the 12 prompts. This component of the task is audio-recorded so that it can be scored by an assessor who is blind to participant's ratings to provide an independent rating of thought valence. Following this, participants are asked to identify a current worry topic, which is recorded by the experimenter. They are then instructed to focus on this topic and worry about it, as they typically would, for 5 minutes. Immediately following the worry period, they again complete the 5-minute breathing focus task in which they are cued to report on their thought content. They again elaborate on their thought content to the researcher. The proportion of distracted thoughts in the second breathing focus period, relative to the first, serves as an indication of worry's interference with attention and the participant's ability to exert attentional control over worry. Two scores were created: one capturing the overall difference in the number of thought intrusions between the two breathing periods, and one capturing the difference in negatively valanced thought intrusions. Lower scores indicate greater thought intrusions in the postworry period relative to the preworry period.

Daily Worry Measures. Participants in the WP and MON conditions were asked to use a phone-based application to 1) Complete event-contingent monitoring of instances of worry throughout the day, and 2) Complete two daily worry questionnaires, one in the morning and one at the end of the day (See Appendices E and F; adapted from Brosschot \& Van Der Doef, 2006 
and Verkuil, Brosschot, \& Thayer, 2007). Worry monitoring and daily worry diaries were completed electronically on a smartphone programmed with the MovisensXS application (MovisensXS, Version 0.4.2437).

Event-Contingent Worry Monitoring. Participants were instructed to access the application (Appendix B) to make a record whenever they noticed an instance of worry throughout the day (i.e., event-contingent worry monitoring). Participants did this by pressing a button in the application, and were prompted to type a brief description of what they were worrying about (Appendix C).

Daily Worry Questionnaires. Participants received a prompt in the evening to complete the daily worry questionnaire in the application (Appendices D and E). During their first visit to the lab, participants in the WP and MON conditions selected a convenient time in the evening and morning to be prompted by the application to complete their questionnaires. The daily worry questionnaire asked participants to report on duration of worry in minutes that day. Participants were also asked to rate how intense their worry was that day on a 7-point Likert scale ranging from not at all intense to very intense. Participants were asked to rate their difficulty disengaging from episodes of worry that day on a 7-point Likert scale ranging from not at all difficult to very difficult. As a compliance check, participants in the MON condition were asked to report whether they completed their worry monitoring throughout the day. Participants in the WP condition recorded whether they completed their worry period that day, and if so, the time and location at which it occurred and its duration in minutes (Appendix F). Participants were also prompted in the morning, at an agreed upon time, to record whether they experienced episodes of worry before bedtime or during the night. If so, they were instructed to log the frequency and duration of nighttime worry (Appendix G). 
Phone Administered PSWQ-PW. On day seven of the 14-day intervention, participants were prompted to complete the PSWQ-PW following their daily worry questionnaire. The participants in the AX-ONLY condition did not complete the PSWQ-PW on day seven because the questionnaire was incorporated into the phone-based questionnaires that were completed in the active conditions, and participant in the AX-ONLY condition did not complete any monitoring between Visits 1 and 2.

Analysis Approach for Worry Diary Data. Four worry measures were created from the daily questionnaire data. A daily worry duration variable was created by summing daily worry duration reported in the evening questionnaire with the late evening and overnight worry duration reported on the next day's morning questionnaire. A daily worry frequency variable was created by summing the number of worry records completed in a day with the number of overnight worry episodes reported in the next day's morning questionnaire. Finally, daily worry intensity and daily worry controllability variables were created based on responses to the corresponding items on the daily worry questionnaire.

Worry monitoring data and daily worry questionnaire data were used for hypothesis testing and as a manipulation check to determine compliance to the interventions in the WP and MON conditions. Worry duration, worry intensity, and worry controllability data were used to test the effect of WP on experience of worry (hypothesis 1). Worry frequency data were used to investigate whether WP leads to stimulus control (hypothesis 4). Completion of daily monitoring and use of the worry period (WP condition only) were used as indices of compliance. Completing worry monitoring and the daily worry questionnaire were also hypothesized to be active components of the WP intervention. That is, noticing and recording naturally occurring periods of worry may have a therapeutic, worry-reducing effect. The inclusion of the MON 
condition allowed for the investigation of the magnitude of therapeutic benefit that comes from monitoring alone.

Worry Postponement Condition. The rationale and instructions for WP were communicated to participants in the WP condition using a script to ensure consistency across participants (See Appendix H). First, participants were presented with a definition of worry, borrowed from Brosschot and Van Der Doef (2006). The rationale for the WP intervention was closely adapted from McGowan and Behar (2013). The rationale used in the present study includes the same content that is described by McGowan and Behar (2013) and this information was edited slightly for fluency. WP instructions followed those outlined by Borkovec, Wilkinson, and colleagues (1983). The same instructions were also used in a recent study of WP (McGowan \& Behar, 2013). Participants who owned an Android smartphone were instructed to download the MovisensXS program. Those who did not own an Android smartphone or who did not wish to use their phone for the study were given a smartphone to use through the duration of the 2-week intervention. Most participants borrowed a phone from the lab (67.2\%), and 32.8\% used their own phone. There was no difference between WP and MON conditions on the proportion of participants who borrowed a phone, $\chi^{2}(1)=.55, p=.46$. The daily worry measures consisted of two components: 1. Event-contingent recording of worry throughout the day when one noticed an episode of worry and 2. Completion of a daily worry questionnaire at the end of the day and in the morning. Instructions for recording worry and completing daily worry questionnaires were reviewed. Along with the instructions, participants selected a daily "worry time," a time in the evening to complete the worry questionnaire, and a time in the morning to complete questions about the previous night's worry. Participants were able to view the WP instructions on the application (Appendix D), and also received a paper version (Appendix H). 
Participants were prompted to ask questions throughout the delivery of instructions, and instructions were clarified as needed. Participants practiced completing each of the worry monitoring forms.

Worry Monitoring Condition. As with WP instructions, worry monitoring instructions were communicated to participants in the MON condition using a script (Appendix I). Participants were provided with the same definition of worry as the WP condition and were given a brief rationale for the positive effects of monitoring worry. They were given a four part set of instructions for worry monitoring, the first step of which was identical to the first step of Borkovec et al.'s (1983) instructions (i.e., learn to identify worrisome thoughts). As in the WP condition, these instructions were made available electronically on the smart phone application (Appendix D) and via paper version. The electronic monitoring of daily worry and the daily questionnaires were reviewed as per procedures in the WP condition. Participants were encouraged to ask questions and practiced completing the forms.

Assessment Only (No Intervention) Control Condition. Participants in the AX-ONLY control condition were given no further instructions following completion of baseline measures. They did not complete daily worry measures, only the outcome measures at the follow-up assessments.

Credibility and Expectancy. The Credibility and Expectancy Questionnaire (CEQ; Borkovec \& Nau, 1972; Devilly \& Borkovec, 2000) includes two three-item scales assessing credibility (e.g., "How logical does the intervention offered seem?”) and expectancy (e.g., "How much do you really feel that the intervention will help you to reduce symptoms?"). Both factors of the CEQ have high internal consistency and good test-retest reliability. Perceptions of treatment credibility and expectancy for change have been found to be related to GAD treatment 
outcomes (Borkovec \& Costello, 1993; Devilly \& Borkovec, 2000). Both the WP and the MON conditions received treatment rationales. The CEQ was administered following delivery of WP or MON instructions. It was included in the study to determine whether different treatment responses were due to group differences in credibility and expectancy.

\section{Procedure}

An experienced doctoral student (KT) assessed eligibility criteria over the phone. The study included three visits to the lab, each spaced out by 2 weeks. Eligible participants were invited to the lab for the first session. Following informed consent, participants completed an attentional control task (N-back), a working memory task (RIG), and a task assessing attentional control over worry (breathing focus task). Participants then completed a general demographics questionnaire assessing age, gender, ethnicity, education level, and employment status, and completed a set of self-report measures (PSWQ-PW, PSWQ, GAD-Q-IV, DASS-21, ISI, SHCQ, RRS, CAQ, MCQ-30, ACS, CAMSR, SHMQ, NPO, and SPSI). Study tasks and questionnaires were completed in the same order at each of the three lab visits, with the exception of the demographics questionnaire and PSWQ which were only administered at the first visit. Participants were randomized using a randomization schedule generated by a computer program (Research Randomizer; Urbaniak \& Plous, 2013) to one of three conditions; WP, MON, or AXONLY. Participants in the active conditions (WP and MON) then received instructions for the 2week intervention period (See Appendices H and I respectively). Instructions were reviewed as per scripts with the participants and clarification was provided when necessary. Participants in the WP and MON conditions also received a set of instructions for completing the phone-based records (See Appendices J and K). Participants in the active conditions (WP and MON) then completed the Credibility and Expectancy Questionnaire (Borkovec \& Nau, 1972). 
Follow-up: Session 2 and Session 3. Two weeks after the first session participants in all three conditions returned to the lab and again completed all self-report measures and tasks. One month from the first session participants returned for a final follow-up assessment, again completing self-report measures and tasks. Participants in the WP and MON conditions were additionally asked to complete an 8-item questionnaire providing feedback on their subjective experience of using the WP intervention (e.g., acceptability, helpfulness etc., see Appendices L and M). Following this, participants were debriefed as to the aims of the study. Participants were compensated $\$ 20.00$ at the first session. $\$ 30.00$ at the second, and $\$ 20.00$ at the third.

This study received ethical approval from the Ryerson University Research Ethics Board. This study was registered prior to starting data collection with ClinicalTrials.gov (Identifier: NCT02806271). 


\section{Results}

\section{Data Screening}

Data were inspected for outliers. First, data for each outcome variable were graphed as box plots. Data identified as outlying on a boxplot were then visually inspected for any abnormalities or errors. Next, z-scores were calculated and inspected. Outliers were identified as those participants having a score that fell more than two standard deviations from the sample mean on a measure. Three outliers were identified on the GAD-Q-IV at each of the three time points, representing the scores of four participants. In all cases, the outlying scores were significantly lower than the mean score. These outlying scores are believed to be an artifact of the GAD-Q-IV's scoring procedure wherein participants who do not endorse item 6 (excessive and uncontrollable worry more days than not) do not complete the second half of the questionnaire, resulting in a lower possible total score for those participants. Further, the participants with low scores on the GAD-Q-IV demonstrated a consistent pattern of lower scores across other outcome measures, suggesting that the scores reflect the participants' true responses and are not the effect or random or erroneous responding. As such, the decision was made to include these outlying scores in the final analyses. Outliers were not detected on any other outcome variable.

\section{Missing Data}

Where participants were missing an item on a questionnaire, that item was replaced by the participant's mean score for all other items on the questionnaire. There were participants missing one item on the SHCQ at visit $1(n=5)$, visit $2(n=5)$, and visit $3(n=3)$.

Due to a programming error with the computer-administered questionnaires, item 15 of the PSWQ-PW ("I worried about projects until they were all done") was not initially included in 
the questionnaire. The error was corrected when detected and resulted in missing data for item 15 across the three visits (Visit $1 ; n=31$, Visit $2 ; n=26$, Visit $3 ; n=23$ ). To address these missing data, two scores were created. First, a score was created using only the 14 of the 15 items that were correctly administered to all participants. Second, missing data were replaced using participant mean imputation, such that missing scores for item 15 were calculated using the average of a participant's responses to the other items. A total PSWQ-PW score was created that included mean imputed values for item 15 for the participants who were missing this item. While mean imputation can risk introducing bias into analyses, it was determined that it would be important to calculate a full scale score for descriptive purposes and to facilitate comparison to samples from other studies.

Analyses were performed using both scores. There were no differences in the results across both sets of scores. Means are presented for the 15 item PSWQ-PW in Table 3. Analyses using the 14 item PSWQ-PW should form the main basis of interpretation, given the lower likelihood of biased estimates.

PSWQ-PW responses were analyzed to determine the internal reliability of the questionnaire and the impact of removing item 15. For participants with complete PSWQ-PW data, the scale had high internal consistency across all three visits ( $\alpha$ ranging from .89 to .92 ) and removing item 15 had a very small impact on the internal reliability (change in $\alpha$ ranging from .002 to .01). Internal reliability was high for the 14 item PSWQ-PW at all three visits ( $\alpha$ ranging from .86 to .90$)$. The high reliability of the 14 item scale, and small impact of removing item 15 provides greater confidence in interpreting the results of the 14 item PSWQ-PW. 


\section{Compliance to Worry Postponement and Monitoring}

In the WP condition, compliance was calculated as the proportion of days that participants indicated on their daily worry questionnaire that they had completed their 30-minute worry time, out of the 14-day intervention period. In the MON condition, compliance was calculated as the proportion of days that participants indicated on their daily worry questionnaire that they had completed their worry records.

Compliance was first investigated for all participants that were randomized to the WP or MON conditions and completed phone monitoring, including those not included in the final sample for analysis $(n=54)$. This was done to characterize the overall compliance in all participants who were randomized to an active condition. Average reported compliance in the WP group was $67.00 \%, s d=25.21$, and in the MON group was $78.57 \%, s d=19.45$. There was no significant difference in compliance between the WP condition and the MON condition, $t(52)$ $=-1.87, p=.07, d=0.52$. Participants in the two conditions also did not significantly differ on their rate of completion of the two daily worry questionnaires or daily completion of worry records.

Next, compliance was analyzed for participants in the WP and MON conditions in the final sample $(n=45)$. An a priori cut score of $50 \%$ compliance was used to determine which participants to include in the final analyses. Compliance was operationalized as the proportion of intervention days on which participants reported they had completed worry monitoring (MON condition) or completed the 30-minute worry period (WP condition). Twenty two participants in the WP condition and 23 in the MON condition demonstrated 50\% compliance or higher. In the final sample, participants in the WP condition had $78.57 \%$ compliance to their intervention on average, participants in the MON condition had $83.23 \%$. 
Compliance rates to intervention components in the final sample are reported in Table 4.

\section{Baseline Differences Between Groups}

One way ANOVAs were used to investigate baseline differences between the three conditions on all outcome variables. There was a significant main effect of condition for the SPSI avoidant problem solving subscale, $F(2)=3.68, p<.05$, with posthoc analyses indicating that participants in the AX-ONLY condition had significantly higher scores than did participants in the MON condition $(p=.009, d=0.92)$. There were no other significant baseline differences between the conditions on any of the outcome measures.

\section{Credibility and Expectancy}

Participants in the WP and MON conditions rated how credible they thought the rationale for their condition's intervention was, and how much they expected the intervention to improve their symptoms of worry and anxiety. Due to a programming error, the credibility items of the CEQ were administered on an 8 point Likert scale (1 to 8$)$ rather than a 9 point scale (1 to 9 ). Participant responses can still be characterized, but scores should not be compared to other samples and should be interpreted in light of this administration error.

There were no significant differences between the WP and MON group on how credible they assessed their assigned intervention to be or how much they expected the intervention to change their worry and anxiety. Participants in both conditions rated the interventions as logical ( $m=6.42, s d=1.22)$. Participants thought the interventions were somewhat likely to reduce their anxiety and worry $(m=5.07, s d=1.56)$, and reported they would be somewhat confident in recommending the intervention to a friend with the same problem $(m=5.12, s d=1.40)$. On average participants thought they would have a $36.19 \%$ improvement in their symptoms of worry and anxiety $(s d=19.04 \%)$ and felt they would have a $30.50 \%$ improvement $(s d=22.18 \%)$. 
Finally, participants indicated that they "somewhat" felt the intervention would be helpful for reducing their worry and anxiety $(m=5.09, s d=1.99)$.

\section{Main Hypotheses}

Data Analysis Plan for Hypothesis Testing. Hierarchical linear modelling was performed for hypothesis testing, including testing the effect of WP on: worry and GAD symptoms (hypothesis 1), cognitive processes related to GAD (hypothesis 2), worry frequency (hypothesis 3), proposed mediators (hypothesis 4) and psychopathology symptoms (hypothesis $5)$.

In the analyses, time (i.e., the day of the study protocol that a data point was collected on) was treated as a level one variable. The time variable was transformed using a loglinear transformation. This transformation fits the data to a pattern of greater initial change that attenuates over time. It was predicted that participants in the active conditions would demonstrate the most change during the 2-week intervention period, and that this change would be sustained at follow-up. As such, the loglinear transformation reflected the pattern of change predicted in the data and allowed for the best test of this prediction.

For analyses that involved all three conditions, the condition variable was dummy coded to allow for comparison between the three conditions. The AX-ONLY condition was set as the reference group, meaning that the models provide estimates for how the WP and MON group changed, relative to the AX-ONLY control group.

Data were predicted to be nested within two levels, the first level including individuals' self-report data across time points, and the second level including condition (WP, MON, AXONLY). Three models were created for each outcome variable: an unconditional mean model, an unconditional growth model, and a conditional growth model. 


\section{Unconditional Mean Model and Intraclass Correlation Coefficient. First, an}

unconditional mean model was created for each outcome variable, entering condition as the only predictor variable. The unconditional mean model allows for the calculation of an intraclass correlation coefficient (ICC). The ICC ranges from 0 to 1 and indicates the proportion of variance in the outcome variable that is due to variance in a second-level factor, in this case, condition (Hayes, 2006). Higher ICC values indicate a greater percentage of variance accounted for by a second-level factor and support the use of HLM to model this variance. However, Hayes has stated that even when an ICC value is close to zero, there can be benefits to using HLM (Hayes, 2006). In the present study, when ICC values were near zero, further models were still tested in order to characterize the effect of time on outcome variables and the time by condition interaction.

There were several outcome variables for which ICC could not be calculated due to very low variance in the level-two variable: condition. This means that there was very little difference between conditions on those outcome variables. When the variance in the level-two variable was approaching zero, the statistical software package used for analyses was not able to calculate it. As such the ICC could not be calculated. The results below specify the variables for which an ICC could not be calculated. Cases in which an ICC could not be calculated indicate that the ratio between variance explained by condition and overall variance in the outcome variable (i.e., the ICC) was near zero. In other words, there was not a significant amount of variance in the outcome variable that could be explained by differences between conditions.

Unconditional Growth Model. Following the unconditional mean model, an unconditional growth model was created to model the effect of time on the outcome variable for the full sample, regardless of condition. 
Conditional Growth Model. Finally, a conditional growth model was created to examine the effects of time, condition, and the interaction between time and condition on the outcome variable. Intercept and time were entered as random factors, meaning that these were allowed to vary between participants.

Analyses were performed using Statistical Package for Social Sciences (SPSS).

Means and standard deviations for the measures used in hypothesis testing, separated by condition, are presented in Table 3, Table 5, Table 6, Table 7, Table 8, and Table 9. Table 10 presents between group effect sizes at the postintervention session and follow-up.

\section{Hypothesis 1: Changes in Worry and GAD Symptoms.}

Results for HLM analyses for Hypothesis 1 are presented in Table 12, Table 13, and Table 14 .

Past week worry. Results are reported here for the 14 item PSWQ-PW. On all analyses, the same pattern of results was present with the 15 item PSWQ-PW. Both sets of results are reported in Table 12.

The unconditional mean model produced an ICC of .04 indicating that $4.49 \%$ of the variance in PSWQ-PW was accounted for by the condition level. The unconditional growth model demonstrated a significant effect of time, $b=-2.21, S E=0.34, t=-6.50, p<.001$. When condition was added to the model, the effect of time remained significant, but there were no significant effects of condition, or time by condition interaction on PSWQ-PW scores. Simple slope analyses were performed to investigate the effect of time in each condition. In each of the three conditions there was a significant effect of time on PSWQ-PW scores (WP; $b=-2.37, S E=$ $0.69 t=-3.44, p<.01, \mathrm{MON} ; b=-2.86, S E=0.61, t=-4.67, p<.001$, AX-ONLY $b=-1.47, S E$ 
$=0.64, t=-2.30, p<.05)$. The baseline to follow-up within-group effect sizes were medium to large in all three conditions (WP; $d=0.74, \mathrm{MON} ; d=1.06, \mathrm{AX}-\mathrm{ONLY} ; d=0.49$ ).

Past week worry in WP and MON Conditions. Both active conditions, WP and MON, completed the PSWQ-PW halfway through the 2 weeks of the intervention period, and therefore had PSWQ-PW scores for four time points: Visit 1 (day 0), midintervention (day 7), Visit 2 (day 14), and Visit 3 (day 28). HLM models were created to examine change across these four time points in the WP and MON groups.

The unconditional mean model produced an ICC of .05, indicating that $5.05 \%$ of the variance in PSWQ-PW scores was accounted for by clustering at the condition level. The unconditional growth model demonstrated a significant main effect of time, $b=-2.21, S E=0.34$, $t=-6.49, p<.001$. When condition was added to create the conditional growth model, time remained significant but there was no main effect of condition or time by condition interaction on PSWQ-PW scores. In both conditions there was a significant effect of time on PSWQ-PW scores across the four timepoints (WP; $b=-2.34, S E=0.68 t=-3.44, p<.01, \mathrm{MON} ; b=-2.85$, $S E=0.54, t=-5.24, p<.001)$.

Results for HLM analyses of past-week worry in the WP and MON condition are presented in Table 13.

GAD Symptoms. The ICC for the unconditional mean model was not calculated because the level two variance was approaching zero and could not be estimated by the statistical software. The unconditional and conditional growth models were run, and there was no significant main effect of time or condition on GAD symptoms as measured by the GAD-Q-IV, and there was no time by condition interaction. 
Worry Diary Responses. The following analyses were performed using only participants from the MON and WP conditions as the AX-ONLY condition did not complete worry diaries. Participants were asked to complete an evening and a morning questionnaire daily for the 14-day intervention period. On average, compliance was high, with participants completing 12.53 evening questionnaires (89.52\%) and 12.64 morning questionnaires (90.32\%). Participants' responses to worry diary questions are summarized in Table 9. Correlations between worry diary data and baseline worry and GAD symptom self-report measures are reported in Table 11. Below, participants' responses to worry diary measures are reported as well as the results of hypothesis testing with diary measures.

Worry Duration. Participants' daily average worry duration ranged from 17.75 minutes to 230.83 minutes, with a mean of 97.33 minutes spent worrying daily $(s d=51.69)$. Average daily worry duration was significantly correlated with baseline PSWQ scores, $r=.47, \mathrm{p}<.01$. Changes in worry duration over time were investigated using HLM. The ICC for the unconditional mean model could not be calculated due to very low level-two variance. There was not a significant main effect of time or condition on daily time spent worrying, and there was no interaction between time and condition.

Worry Controllability. Participants rated their daily average ability to stop worrying once they started as $3.10(s d=.72)$ on a Likert scale that ranged from 0 (not at all difficult) to 6 (very difficult). Average daily ability to disengage from worry was significantly correlated with baseline PSWQ scores, $r=.44, p<.01$.

The ICC for the unconditional mean model could not be calculated due to very low leveltwo variance. There were no main effects of time or condition and no interaction between time 
and condition on participants' ratings of how difficult it was for them to disengage from their worry.

Worry Intensity. On average, participants identified the daily intensity of their worry as $3.27(s d=.62)$ on a Likert scale that ranged from 0 (not intense at all) to 6 (very intense).

Average daily worry intensity was significantly correlated with baseline PSWQ scores, $r=.37, p$ $<.05$.

The ICC for the unconditional mean model could not be calculated because of very low level-two variance. There was a significant effect of time found on daily worry intensity, $b=$ $0.14, S E=0.07, t=-2.06, p<.05$. When condition was added to the model, there was no longer a main effect of time on participants' daily rating of how intense their worry was, and there was no interaction between time and condition. Given that there was a significant effect of loglinear time, worry intensity scores were examined using piecewise analysis to determine if intensity scores changed at a different rate during the first week of monitoring compared to the second week. Results showed no significant effect of time in week 1 or week 2.

Summary of Hypothesis 1 Results. In summary, participants in all conditions demonstrated a significant reduction in past-week worry across the duration of the study, with no significant differences between conditions. No changes in GAD symptoms were observed across the course of the study. Participants in the WP and MON condition had a significant decrease in the intensity of their worry across the 2-week intervention period, as recorded in their daily worry diaries.

\section{Hypothesis 2: Changes in Cognitive Processes Related to GAD.}

Results for HLM analyses for Hypothesis 2 are presented in Table 15, Table 16, and Table 17. 
Mindfulness. First, changes in mindfulness were examined based on participant scores on the SHMQ. The unconditional mean model produced and ICC of .01, indicating that $1.26 \%$ of the variance in SHMQ scores was accounted for by clustering at the condition level. The unconditional growth model demonstrated a significant main effect of time, $b=0.70, S E=0.29$, $t=2.41, p<.05$. When condition was added to create the conditional growth model, there was no longer a significant effect of time. Further, there was no main effect of condition or time by condition interaction on SHMQ scores. Simple slope analyses were used to investigate the effects of time in each condition. When each condition was examined independently they no longer demonstrated a significant effect of time on SHMQ scores.

Next, scores on the CAMS-R were used to investigate changes in mindfulness. The ICC could not be calculated for the CAMS-R due to very low level-two variance. There were no main effects of time or condition or time by condition interaction effect on CAMS-R scores.

Negative Problem Orientation. The ICC could not be calculated for participants' NPO scores due to very low level-two variance. No main effects of time or condition were found, and no time by condition interaction was found for participants' attitudes toward problem solving.

Problem Solving Style. Four problem solving styles were examined: positive problem orientation (SPSI-PPO), rational problem solving (SPSI-RPS), impulsive and careless problem solving (SPSI-ICS), and avoidant problem solving (SPSI-AS). An ICC could not be calculated for SPSI-ICS scores due to low variance in the level-two variable. For the other problem solving styles, the ICCs were .06 (SPSI-PPO), .06 (SPSI-RPS), and .09 (SPSI-AS). There were no significant main effects of time or condition or time by condition interaction effects for scores on positive problem solving, rational problem solving, or impulsive careless problem solving. No main effect of time was detected for SPSI-AS scores, however, when all conditions were added 
into the conditional growth model, there was a significant effect for the MON condition, $b=$ 3.49, $S E=1.26, t=-2.78, p<.01$. This indicates that scores for the MON condition, regardless of timepoint, were significantly lower than were those for the AX-ONLY condition. This is consistent with the results of the ANOVA conducted to test for baseline differences, which demonstrated baseline between-group differences in SPSI-AS scores. There was no interaction between time and condition, suggesting that the scores of the MON or WP conditions did not change at a significantly different rate than those of the AX-ONLY condition.

Cognitive Avoidance. The ICC for CAQ scores could not be calculated due to very low variance in level-two. There was no significant effect of time or condition and no condition by time interaction on CAQ scores.

Attention Control. Three indices of attentional control were examined: a self-report measure of attentional control (ACS), and two behavioural tasks, the RIG and the N-Back. The ICC for ACS scores could not be calculated due to low level two variance. No main effects of time or condition, or time by condition interaction were found for ACS scores.

The RIG task produces two related scores that capture the randomness of a participant's responding: A redundancy score (RIG-R) and a random number generation score (RIG-RNG). For both scores, higher scores indicated lower working memory capacity. The ICC for the unconditional mean model of RIG-R scores was .03, indicating that $3.23 \%$ of variance in scores was due to clustering at the condition level. The unconditional growth model demonstrated a significant effect of time, $b=1.08, S E=0.44, t=2.43, p<.05$, indicating that all participants on average, regardless of condition, had a significant increase in scores over time. The effect of time no longer remained significant when condition was added to the model. In the conditional growth model, the slope of the WP group was significantly different than that of the AX-ONLY 
group, $b=2.15, S E=1.07, t=2.01, p<.05$. This indicated that the RIG-R scores of the WP condition increased at a greater rate than did those in the AX-ONLY condition. Simple slope analyses demonstrated that WP was the only condition that demonstrated a significant change in RIG-R scores over time, $b=1.86, S E=0.59, t=3.15, p<.01$.

The ICC for the RIG-RNG unconditional mean model was .02, indicating the $2.08 \%$ of variance in scores could be explained at the condition level. There were no main effects of time or condition. In the conditional growth model, the slope of the MON condition was significantly different from that of the AX-ONLY condition, $b=0.02, S E=0.01, t=2.02, p<.05$, indicating that the RIG-RNG scores in the MON condition changed over time at a different rate than those in the AX-ONLY condition. Simple slope analyses demonstrated that the WP condition was the only condition that demonstrated significant change in RIG-RNG scores over time, $b=0.01, S E$ $=0.01, t=2.12, p<.05$.

The N-back task produces three scores, indicating participant performance in the 1-back, 2-back, and 3-back condition. The ICC for the 1-back condition was .04 indicated that $3.77 \%$ of the variance in scores was accounted for by condition. An ICC could not be calculated for the 2back condition and 3-back condition due to very low level-two variance. For N-Back 1-back scores, no significant effects of time, condition, or time by condition interaction were found. When N-back 2-back scores were modeled, a significant main effect of time was demonstrated in the unconditional model, indicating that scores were increasing across time, regardless of condition, $b=0.02, S E=0.003, t=5.14, p<.001$. There was also a significant main effect of time demonstrated for N-Back 3-back scores, $b=0.02, S E=0.003, t=5.01, p<.001$, indicating improving performance over time averaged across conditions. For N-Back 3-back scores, the effect of time remained when condition was added to the model, $b=0.02, S E=0.01, t=2.94, p$ 
$<.01$. These results indicate that N-back 2-back and 3-back scores improved on average for participants over the course of the study. For all N-back variables, there were no main effects of condition or time by condition interaction. Simple slope analyses demonstrated that all three conditions had significant change in N-back 2-back and 3-back scores over the course of the study.

Summary of Hypothesis 2 Results. In summary, on average across conditions there was a significant increase in mindfulness over time. However, this increase was only demonstrated on one of two mindfulness measures, and not demonstrated within conditions when simple slopes were analyzed. No significant effects of time or condition were demonstrated on negative problem orientation, problem solving style (with the exception of baseline differences on SPSIAS), cognitive avoidance, or self-reported attentional control. On one behavioural measure of attentional control (RIG), one index showed a significant increase in scores over time, reflecting lower working memory capacity. This RIG trend was more pronounced in the WP condition on one index, and more pronounced in the MON condition on another. On two N-Back indices, participants in all conditions showed significantly better performance over time.

\section{Hypothesis 3: Changes in Worry Frequency and Stimulus Control.}

Results for HLM analyses for Hypothesis 3 are presented in Table 18.

Change in worry frequency, operationalized as number of daily worry episodes recorded, was examined as an index of worry coming under stimulus control. Participants in the WP and MON conditions were asked to record each independent worry episode they experienced, daily, for 14 days. On average participants completed at least one worry record on $11.49(s d=3.15)$ of the 14 days $(82.06 \%)$. Participants recorded an average of $4.48(s d=3.01)$ worry episodes daily. There was no correlation between participants' average number of daily worry episodes and their baseline PSWQ scores, $r=.009, p=.952$. 
HLM was used to model change in worry frequency over the intervention period. The ICC for worry episodes could not be calculated due to very low level-two variance. In the unconditional growth model, a significant effect of time was demonstrated, indicating that frequency of worry episodes decreased over the course of the intervention period, on average across both conditions, $b=-1.04, S E=0.26, t=, p<.001$. When condition was added to the model, the effect of time remained significant, $b=-0.80, S E=0.38, t=-2.09, p<.05$, and there was no significant main effect of condition or condition by time interaction for worry frequency.

\section{Hypothesis 4: Proposed Mediators.}

Results for HLM analyses for Hypothesis 4 are presented in Table 19.

Metacognitive beliefs about worry's controllability, and attentional control over worry were hypothesized to be potential mediators of change in worry in the WP intervention. Metacognitive beliefs were measured using the MCQ "Controllability" subscale. Attentional control over worry was measured using the breathing focus task. Two scores from the breathing focus task (BFT) were used: 1. The difference in total thought intrusions between two 5-minute mentation periods, before and after a period of worry (BFT-Total), and 2. The difference in negative thought intrusions in those same two mentation periods (BFT-Negative). Change in these potential mediators was modelled using HLM.

Metacognitive Beliefs. The ICC could not be calculated for MCQ-Controllability scores because of very low level-two variance. There were no significant main effects of time or significant time by condition interaction on MCQ-Controllability scores.

Attentional Control Over Worry. Participants were prompted to report their thought content and valence at 12 points during two 5-minute breathing focus periods. At the first visit, there was no significant difference in the total number of thought intrusions (irrespective of 
valence) participants reported before and after the worry period $(t=-1.35, p=.18$, Preworry intrusions; $M=6.04, S D=2.32$, Postworry intrusions, $M=6.40, S D=2.57)$. However, participants did report significantly more negative thoughts in the postworry period than the preworry period, suggesting that there was a change in the proportion of their thought intrusions that were negative $(t=-4.40, p<.001$, Preworry negative intrusions; $M=2.17, S D=2.11$, Postworry negative intrusions, $M=3.32, S D=2.48$ ).

The unconditional mean model of BFT-Total scores produced an ICC of .01, indicating that $1.20 \%$ of variability in scores was due to clustering at the condition level. The ICC for BFTNegative could not be calculated due to very low level-two variance. No significant main effects of time or cognition, or time by condition interaction were demonstrated for either BFT measure.

As there were no significant changes in the proposed mediator variables, mediation analyses were not performed.

\section{Hypothesis 5: Changes in Other Clinical Symptoms.}

Results for HLM analyses for Hypothesis 5 are presented in Table 20.

Symptoms of Depression. Depression symptoms were measured using the DASS-21 Depression subscale. The ICC for the unconditional mean models was .03 , indicating $2.93 \%$ of the variance in DASS-21 depression scores was due to level two clustering. There was a significant main effect of time, but this was only present in the unconditional growth model, $b=$ $-0.34, S E=0.14, t=-2.32, p<.05$, indicating that for all participants, on average, there was a significant decrease in depressive symptoms over the course of the study. In the conditional growth model there were no main effects of time or condition or time by condition interaction effect on DASS-21 depression scores. The significant effect of time was followed up with simple 
slope analyses. When the effect of time was examined separately in each of the three conditions, there was no significant effect of time on depression symptoms demonstrated.

Symptoms of Anxiety. The DASS-21 anxiety subscale was used to measure changes in anxiety symptoms. The ICC could not be calculated for DASS-21 anxiety scores due to very low level-two variance. There was a significant main effect of time in the unconditional growth model for DASS-21 anxiety scores, $b=-0.37, S E=0.13, t=-2.98, p<.01$, indicating that participants' anxiety symptoms decreased, on average, over the course of the study, regardless of which condition participants were in. In the conditional growth model there was no longer a significant main effect of time, and there was no significant effect of condition or time by condition interaction. Simple slope analyses were used to examine the effects of time in each of the three conditions and only the MON condition demonstrated a significant effect of time on symptoms of anxiety, $b=-0.54, S E=0.20, t=-2.77, p<.05$.

Subjective Health Complaints. The unconditional growth model for SHCQ scores produced an ICC of .02 , indicating $2.42 \%$ of variance in scores was due to clustering at the condition level. In the subsequent models, there were no main effects of time, condition, or time by condition interaction on SHCQ scores.

Symptoms of Insomnia. An unconditional growth model was run for scores on the ISI, with an ICC of .01, demonstrating $1.44 \%$ of variance in scores was due to condition clustering. There were no significant main effects of time or condition, and no condition by time interactions on ISI scores.

Rumination. Changes in rumination were investigated as an exploratory question. An ICC could not be calculated for RRS scores due to very low level-two variance. There were no 
significant main effects of time or condition, and no significant interaction effects on RRS symptoms.

Summary of Hypothesis 5 Results. In summary, all participants on average had a reduction in depression symptoms over the course of the study, but when simple slopes were examined there were not significant within condition effects in any condition. Participants on average had a reduction in anxiety symptoms and when conditions were examined individually this effect only remained significant in the MON condition. There were not significant effects of time or condition or time by condition interactions on subjective health complaints, insomnia symptoms, or rumination.

\section{Clinically Significant Change}

Clinically significant change in symptom measures was examined as part of the planned analyses. Scores on PSWQ-PW, GAD-Q-IV, DASS-21 Depression, DASS-21 Anxiety, ISI, SHCQ, and RRS were examined. Clinically significant change was operationalized as the proportion of participants whose Visit 3 scores were at least two standard deviations below the baseline sample mean (Jacobson et al., 1984). Results are presented in Table 21. The percent of participants achieving clinically significant change on symptom measures ranged from $0 \%$ to $22.7 \%$. Chi-square tests indicated that there were no differences between conditions in the proportion of participants achieving clinically significant change on any symptom measures.

\section{Feedback}

Participants in the two intervention conditions, WP and MON, were administered a feedback questionnaire at the end of the third visit, after completing all experimental measures. Participants responded to rating scales and were given the opportunity to write feedback. It should be noted that this questionnaire was not administered to participants who did not attend 
the third visit. The questionnaire was administered in order to assess participant reactions to completing the interventions. No hypotheses were advanced regarding the feedback questionnaire. Responses from all participants who completed the study were included in analyses as it was determined to be important to include participants who had good compliance to the intervention and participants who did not meet the $50 \%$ compliance criterion. Results of the questionnaire are summarized in Table 22.

Participants were asked if they continued to use the intervention over the 2-week period between visit 2 and visit 3 . In the WP condition, the modal response was "Continued to use the intervention a few times" (42.31\%), and in the MON condition most participants reported that they did not continue to use the intervention (63.64\%). Participants rated how helpful the intervention was, how easy to use it was, and how confident they would be in recommending the treatment. The only significant difference between conditions was in ease of use, with MON participants rating the intervention as significantly easier to use, $t(39)=-2.17, p<.05, d=0.70$.

Participants in the WP condition were asked how they primarily spent their worry time. Participants reported they spent their worry time "Worrying about concerns that came up during the day" (45.8\%), "Problem solving concerns that came up during the day"(37.5\%), and "Other" (e.g., "Feeling distracted while worrying about concerns"; $12.5 \%$ ). One participant (4.2\%) reported that they did not use their worry time.

Qualitative responses from the feedback questionnaire were not formally analyzed. They are included in Appendices $\mathrm{N}$ and $\mathrm{O}$. These responses are thought to be useful for the purpose of understanding how participants reacted to being asked to track and/or postpone their worries, and for the purpose of generating future ideas for refining worry monitoring or WP interventions. The qualitative responses were generally positive with the majority of participants identifying 
useful qualities in the interventions that are theoretically in line with how the interventions are expected to work. For example, participants in WP reporting that postponing their worries freed their attention to focus on what they were doing, and participant in MON reporting that tracking increased their awareness of how much time they were worrying. 


\section{Discussion}

Generalized anxiety disorder is a disorder characterized by excessive worry that results in a high degree of physical and mental health burden for those affected. Untreated, GAD has a chronic unremitting course. Following evidence-based treatment, a significant portion of people with GAD do not achieve remission (Fisher, 2006; Hanrahan, Field, Jones, \& Davey, 2013). A promising avenue for improving treatment for GAD is to examine the efficacy of the treatment components that make up evidence-based multicomponent treatments such as CBT. WP is a strategy that is commonly included in treatment packages for GAD (e.g., Borkovec, 2006; Newman \& Borkovec, 2002; Rygh \& Sanderson, 2004; Wells, 2006), and commonly applied by clinicians in treating GAD (Szkodny et al., 2014). WP was first described over 30 years ago. The limited research on WP suggests that it is a promising strategy for reducing worry and associated mental and physical symptoms. However, despite its promise and widespread use, there are no published studies to my knowledge that have investigated WP's efficacy in treating worry in people with GAD.

The primary aim of the present study was to test the efficacy of WP in people with GAD. In addition to there being a gap in knowledge regarding the intervention's efficacy, little is known about the mechanisms of action of WP. Two different rationales for WP have been presented in the treatment literature. The first, is that WP leads to stimulus control over worry; that is, that it reduces the generalized quality of worry by, over time, limiting the external stimuli

it is associated with (Borkovec, Wilkinson, et al., 1983). The second, is that WP has its effects by reducing the metacognitive belief that worry is uncontrollable, and thus leads to more selfinitiated efforts to interrupt worry (Wells, 2006). Neither stimulus control nor metacognitive 
beliefs have been tested as potential mechanisms of action for WP. This study tested if WP is efficacious for reducing worry, what processes best account for this effect.

The WP intervention is composed of several "mini skills," including monitoring worry patterns, practicing disengaging from worry, attending to present moment experience, restricting worry duration, focused time spent worrying, and active problem solving. Given these different components of the treatment, it is plausible that WP has broad impact on cognitive processes related to worry. The present study specifically examined whether WP leads to changes in cognitive avoidance, attentional control, mindfulness, and problem solving attitudes and styles.

There are several limitations to the current research literature on WP, above and beyond the fact that the intervention has not been studied in people with GAD. The mechanism of action of WP is unknown, as are the effects on outcomes other than symptoms. It is unknown whether WP is superior to monitoring worry alone as current research has had mixed results (Jellesma et al., 2009; Verkuil et al., 2011). Directly comparing WP to a monitoring intervention and to a control condition (as was done in this study) allows for more precise conclusions about the additive effects of WP's components. Only one study (Verkuil et al. 2011) has examined the effects of WP beyond an immediate postintervention assessment. As such, the short and long term effects and maintenance effects of WP are unknown. Finally, pathological worry is considered to have many qualities, in that it is excessive in its frequency, duration, and intensity, and is described as uncontrollable. Given this, more needs to be known about which elements of pathological worry, if any, WP effects in people with GAD.

This study compared the effects of a 2-week WP intervention with people with GAD to 2 weeks of worry monitoring alone, and to a 2-week, no intervention control condition. The majority of studies on WP have used intervention periods ranging from 6 days to 2 weeks 
(Brosschot \& Van Der Doef, 2006; Jellesma et al., 2009; McGowan \& Behar, 2013; Versluis et al., 2016; Verkuil et al., 2011). Changes in worry, GAD symptoms, cognitive processes related to worry, and symptoms related to GAD were examined at baseline, immediately post 2-week intervention, and at a 2-week follow-up.

\section{Worry and GAD symptoms}

All three conditions demonstrated a medium to large reduction in self-reported past-week worry over the course of the study. However, in the WP condition, the magnitude of the intervention's effect on self-reported worry $(d=0.71)$ was much less than what has been reported in previous studies of WP with people high in trait worry $(d=1.81$; McGowan \& Behar, 2013). In the present study, there were no significant changes in any of the conditions in selfreported GAD symptoms. Participants in the active conditions additionally reported their daily worry using a phone based application. Participants in the WP and MON conditions demonstrated a reduction in the intensity of their worry over the 2-week intervention period as reported in the daily worry diary. Neither WP nor MON resulted in significant changes in selfreported worry duration, or worry controllability.

\section{Cognitive Processes Related to GAD}

The impacts of WP on cognitive processes related to GAD was examined. Specifically, it was hypothesized that WP would lead to significant increase in mindfulness and attentional control, and a significant decrease in negative problem orientation and cognitive avoidance, relative to control conditions. This hypothesis was unsupported. All participants, on average, evidenced an increase in mindfulness over the course of the study, although this was only demonstrated on one of the two mindfulness measures included. Participants did not show change on a general self-report measure of mindfulness, the CAMS-R. The measure that 
participants did demonstrate significant reduction on over the course of the study was the SHMQ, which assesses mindful reactions to upsetting thoughts or images. This is notable, as worry can be conceptualized as an avoidant reaction to upsetting thoughts or images, in opposition to a mindful reaction.

Attentional control was assessed using three measures; a self-report measure, and two behavioural measures, the RIG and the N-Back tasks. The results were mixed for changes in attentional control across conditions. There were no significant changes found in self-reported attentional control. The RIG task which is intended to measure working memory capacity demonstrated different patterns of results on its two indices. For each of the RIG indices, higher scores indicate less random responding and therefore less working memory capacity. Baseline RIG scores on both indices were very similar to scores reported previously for a diagnosed GAD sample (Tallon et al., 2016). One RIG index demonstrated an overall increase in scores among the three conditions, with the WP condition showing a greater rate of increase than the AXONLY condition. The other index demonstrated no overall change in scores, but a difference between the MON and AX-ONLY conditions, with the MON condition showing a significantly greater rate of increase. These results run counter to the predicted results, in which the WP and MON conditions were expected to show a greater increase in working memory capacity than the AX-ONLY condition. There are no theoretical explanations for why participants would demonstrate a decrease in working memory over the course of the study. One methodological explanation could be that participant motivation and effort on the RIG task could have declined over the course of the study as completing the task can be unstimulating, unlike the N-Back which is quite interactive and provides immediate feedback. 
The N-back task produces three scores reflecting its three, progressively more difficult, task conditions. On the 2- and 3-back conditions of the task, participants across conditions showed significant improvement over the three study visits. It is possible that this change reflects a training effect. Participants would have had increased familiarity with the task across the three visits and this may have translated into improving performance. Further the N-Back provides in the moment feedback on performance which likely reinforces good performance, possibly leading to improvement over time.

No changes were found in participants' negative problem orientation, problem solving style, or tendency for cognitive avoidance.

\section{Stimulus Control}

The stimulus control theory of WP proposes that the intervention works by bringing worry under greater stimulus control (Borkovec, Wilkinson, et al., 1983). That is, worry is thought to be generalized because it is cued by a wide range of internal and external stimuli. WP is proposed to gradually reduce the stimuli that worry is associated with and therefore reduce spontaneous occurrences of worry. If WP has its effects through stimulus control one would expect to see fewer episodes of worry over time. This hypothesis was tested in the present study by having participants in the two active conditions complete event contingent monitoring of their worry episodes. Participants were asked to record a brief description of their worry each time they noticed they were worrying. The number of worry episodes that participants reported significantly decreased over the 2-week intervention period, and there was no difference between the two active conditions. This could be interpreted to show that WP and worry monitoring lead to a reduction in the frequency of worry. However, several other possibilities should be considered as well. There are reasons to doubt the reliability of the worry frequency measure. 
There was a very large range in the number of worry episodes participants reported. Worry frequency was the only worry diary measure that did not correlate with validated self-report measures of worry and GAD symptoms. Therefore, it is possible that the number of episodes recorded is not an accurate reflection of participants' experience of worry. The worry episode data relies on many factors including participants noticing that they are worrying, remembering to complete a worry record, and then completing the worry record. It is possible that this measure is sensitive to participant factors other than worry frequency such as participant motivation and conscientiousness. Another possible explanation for the reduction in worry frequency ratings over the 2 weeks is that participant motivation and vigilance for recording worry episodes may have waned over the 2-week intervention period. Therefore, based on the present findings and the shortcomings of the worry frequency measure, it is unclear whether WP or monitoring led to true reductions in worry frequency over the intervention period.

\section{Proposed Mediators}

No studies to date have examined the mechanisms of action of WP. Metacognitive therapy proposes that WP has its effects by changing the metacognitive belief that worry in uncontrollable (Wells, 2006). Metacognitive beliefs about worry's controllability did not change significantly over the study in any of the study conditions. It should be noted that the rationale and instructions for WP in this study were consistent with the stimulus control model of WP (i.e., Borkovec, Wilkinson, et al., 1983), and that metacognitive therapy uses different instructions for administering WP. In metacognitive therapy, WP is employed as a behavioural experiment to test the belief that worry is uncontrollable. The therapist has a client rate their belief that worry is uncontrollable before and after the experiment, as well as repeating the experiment as necessary until there is change in this belief. It is possible that if the intervention had been implemented 
with this experimental frame, participants may have attended more to evidence against the belief "worry in uncontrollable," and more change would have been observed in the belief. As such, the present study is not a pure test of WP as applied in metacognitive therapy, and this may partly account for the lack of observed changes in metacognitive beliefs.

I further proposed that WP could have its effects through increase attentional control over worry. Attentional control over worry was examined using a behavioural task, the breathing focus task, that measures thought intrusions and their valence before and after a 5-minute worry period. No changes in thought intrusions were found over the course of the study in any of the study conditions.

The breathing focus task also provides information about the effect of worry on thought intrusions in people with GAD. At baseline, there was no difference in the number of overall intrusions participants reported before and after the worry period. Participants reported that they were able to focus on their breathing about half of the time. This suggests that participants found it difficult to control their thinking when asked to focus on their breathing. However, there was no healthy control comparison group, so this finding may reflect typical performance.

Participants did report a higher proportion of negative thought intrusions following the worry period, on average having approximately one more intrusion, that they characterized as negative, following the worry period. A previous study using the same breathing focus protocol demonstrated a similar increase in negative thought intrusions following a period of worry (Hayes, Hirsch, Krebs, \& Mathews, 2010). The present findings could suggest that worrying has a greater impact on thought valence than thought process. That is, worry lead to more negative thoughts, but not more thought intrusions. 


\section{Symptoms Related to Generalized Anxiety Disorder}

Previous studies on WP have demonstrated that the intervention has effects on physical and mental health symptoms related to GAD including depressive symptoms, anxiety, insomnia, and health complaints (Brosschot \& Van Der Doef, 2006; Jellesma et al., 2009; McGowan \& Behar, 2013; Verkuil et al, 2011; Versluis et al., 2016). The present study found a significant reduction in depressive symptoms and symptoms of anxiety over the course of the study, with no differences between conditions. Within conditions, the changes in depression and anxiety symptoms were small to medium in magnitude, and with the exception of anxiety symptoms in the MON condition, the effects were not significant. Despite the statistically significant change in depression and anxiety symptoms on average, symptoms stayed within the moderate range of severity from baseline to follow-up for all three conditions, suggesting that the change was not clinically significant. Contrary to previous studies there were no changes across the study in symptoms of insomnia or in the number of subjective health complaints.

\section{Clinically Significant Change}

A previous study of WP in people with high trait worry demonstrated a large percentage of participants in the WP condition evidencing clinically significant change, as indicated by the proportion of participants whose post intervention score was more than two standard deviations below the preintervention sample mean (McGowan \& Behar, 2013). In the present study, few participants met this criterion for clinically significant change on measures of worry, GAD symptoms, and related symptoms. Consistent with the results of hypothesis testing, there were no differences between the conditions in the proportion of participants whose post test scores reflected a clinically significant change. 


\section{Participant Reactions to the Intervention}

It is important to consider how participants evaluated the interventions they were assigned to in order to help understand the pattern of results. Participants rated the WP and MON interventions as equally credible. Participants expected the interventions would be "somewhat" helpful. Participants appeared to have a modestly positive belief that the interventions would be helpful for reducing their worry and anxiety. It is possible that this mild expectation impacted the effort participants put into the intervention.

Participants also provided feedback on the interventions at the end of the study. The only difference between the WP and MON conditions' ratings were on ease of use, with the MON condition rating their intervention as significantly easier to use than the WP condition. This difference makes sense given the increased demands of the WP intervention, which requires participants to dedicate 30 minutes at the same time and place daily for 2 weeks. It is possible that it was easier for MON participants to fully engage in their intervention and this may account for some of the equivalence between participant results regardless of the fact that the WP intervention was more involved

\section{Explanation of Null Findings}

This study represents one of the most rigorous and well-controlled studies of WP to date. The results of this study are inconsistent with previous studies demonstrating WP's efficacy in unselected samples and samples of people high in trait worry (Brosschot \& Van Der Doef, 2006; Jellesma et al., 2009; McGowan \& Behar, 2013; Verkuil et al, 2011). Given the inconsistency of this study's results with previous research, several possibilities need to be considered to explain the study's findings. 
The first way to interpret the findings is to conclude that WP does not have a significant effect on worry and related symptoms in people with GAD. Given that there were no significant differences between participants in the WP condition and participants in two control conditions, it is possible that this intervention simply does not lead to significant or meaningful change in people with GAD. This is the first study to use WP in a diagnosed sample of people with GAD. It could be that WP is not efficacious for people with a higher severity and chronicity of worry.

Further to the possibility that WP is not efficacious for people at higher severity levels of worry, it is possible that WP, as it was delivered in the present study, was not optimal for this population. In current clinical practice WP is typically used as one component in a multicomponent treatment for GAD. It is possible that WP is most effective when it is grounded in a larger treatment rationale or is presented in treatment after certain foundational skills have been developed such as learning to monitor worries, or greater understanding about the function of worry.

In this study to ensure standardization WP was delivered using a script. The script used in this study was consistent with how the intervention was delivered in other studies (McGowan \& Behar, 2013; Brosschot \& Van Der Doef, 2006). It is possible that delivering the intervention in a more interactive or Socratic way, that allows the person to more deeply understand the rationale, could enhance motivation and engagement with the exercise. However, there is initial evidence for the efficacy of treatments for GAD that have minimal clinician contact, for example self-help or internet based treatments (Cuijpers et al., 2014; Lewis, Pearce, \& Bisson, 2012). In two early studies of WP there were no differences in findings when participants had a weekly check in call and when they did not (Borkovec, Wilkinson, et al., 1983). Therefore, it is unknown 
whether more therapist contact, or greater personalization of the WP intervention would lead to enhanced outcomes.

The population that this study's sample came from is more clinically severe than the populations that WP has been tested in previously. It is possible that participants required a higher dose of WP to demonstrate an effect, and that the 2-week intervention period was too brief. However, other studies have employed comparably brief interventions in people with GAD, or high in GAD symptoms, and demonstrated significant changes in worry (e.g., LaFreniere \& Newman, 2016; Eagleson, Hayes, Mathews, Perman, \& Hirsch, 2016). For example, one study had a sample with comparable baseline trait worry and found a significant decrease in worry following a 1-week intervention where participants practiced generating positive outcomes in response to worry (Eagleson et al., 2016). This demonstrates that brief interventions for worry can be efficacious even in participants with high baseline trait worry.

In sum, it is possible that the effects of WP are enhanced when it is delivered in the context of a larger treatment package or that a larger dose of the treatment is needed for people at a high severity of worry.

One limit to making conclusions from this study about WP's efficacy is that the full extent of participants' compliance to the multiple parts of WP is unknown. Participants were asked to indicate in daily questionnaires whether they had used their worry period. Participants did report relatively high adherence to the worry period, but there is no way to verify this. It is possible that participants indicated they did complete the worry period, due to impression management, when they may have not fully adhered to the intervention. Further, it is unknown whether participants persisted in attempts to disengage from and postpone worry. Reported compliance to the worry period was slightly lower in the present study $(78.57 \%$ percent of days 
completed on average) than in a previous study (87.5\%; McGowan \& Behar, 2013). In the present study, this translates to approximately 1 day less of reported use of the worry period, compared to compliance reported in a previous study. As such, it is possible that in this study participants in the WP group did not fully engage with the unique aspects of the WP intervention, and that this explains the lack of difference between the WP and MON group.

Participants in all three conditions demonstrated a significant reduction in past week worry over the study period. Given that worry is believed to be stable in GAD in the absence of treatment, it is possible that some element of taking part in the study led to a reduction in worry. While the AX-ONLY condition was intended as an "inactive" control condition, there are several elements of the condition that could have possibly led to a change in participants' worry.

Participants in the AX-ONLY condition completed a clinical interview about their worry and its impact on their functioning and filled out many questionnaires about their worry and associated symptoms at three intervals over the course of a month. Participants also completed behavioural tasks that may have raised their awareness about their worry patterns. For example, the breathing focus task involves participants reporting a detailed description of a current worry which the experimenter then paraphrases back to them. Further, in the breathing focus task participants are asked to spend time focusing on their worry and to practice turning their attention away from worry during the breathing focus periods. It is possible that the breathing focus tasks acts as a brief mindfulness intervention by demonstrating how to disengage from worry. In fact, the full sample showed an increase in mindful attitudes towards intrusive thoughts and images. Given all this, it is possible that the AX-ONLY condition had a significant effect on worry and that the MON and WP conditions were not powerful enough to demonstrate an effect over and above the effects of merely taking part in a worry study. 
This is one of the first studies to examine the effects of WP beyond an immediate postintervention follow-up. One study found group differences between participants who had completed WP as a pretreatment before a work stress group treatment, and those who had not at a 3-month follow-up (Verkuil et al., 2011). More information is needed on how the effects of WP are maintained long term. Given that participants in all study conditions in the present study demonstrated a reduction in worry, it would be interesting to know if these effects were maintained to an equal degree over a longer period of time.

The findings of the present study are in line with Hirsch and Mathew's (2012) cognitive model of pathological worry. Hirsch and Mathews (2012) argue that pathological worry occurs as a result of both bottom up and top down attentional factors. For example, automatic processes such as an attention bias to threat make it more like that worry will be triggered for people with pathological worry. Reduced attentional resources due to worry and metacognitive beliefs about worry are proposed to have a top down effect that further perpetuate a worry episode. Hirsch and Mathews predict that interventions that focus purely on top-down control of worrying are unlikely to have a lasting impact on worry as they do not alter the bottom up processes, such as attention or interpretation biases, that trigger new worry episodes. They identify WP as one such "top down" intervention that is likely to have limited effectiveness for pathological worry. WP is proposed to be a top down intervention because it requires participants to exert effortful control over worry episodes by redirecting their attention to the present moment each time they worry. Hirsch and Mathews propose that "top down" interventions like WP should be combined with techniques aimed at re-training processing biases in order to be maximally effective. This prediction is mostly consistent with the current findings. Although participants in the WP condition did show reduced past week worry and reduced worry intensity, these changes were 
not different than those demonstrated in the control conditions, and the WP intervention did not impact a broader range of symptoms and cognitive processes related to worry. Therefore, one possible reason for why WP did not lead to significantly greater change in worry relative to other conditions is that it did not impact bottom up cognitive processes that perpetuate worry.

\section{Worry Diary Responses}

The present study contributes interesting new information about the daily worry experiences of people with GAD. Participants in the WP and MON conditions completed two daily questionnaires about their worry, and recorded individual episodes of worry. One interesting insight from the diary data is that participants' daily record of their worry reflects a lower severity of worry than is typically described when participants are asked to estimate their daily time spent worrying. For example, participants' average duration of worry within a 24-hour period was just slightly more than an hour and a half. The amount of time spent worrying is much less than what participants reported in the structured interview, typically reporting they spend "most of the day" worrying. Further participants rated the intensity and controllability of their worry, on average, in the middle of a 7-point Likert scale, indicating that their worry was "somewhat" intense and "somewhat" difficult to disengage from. It is possible that the discrepancies between participants' subjective reports and their daily diary data reflects a difficulty in accurately measuring daily worry. However, this discrepancy may indicate that some of the distress about worry experienced by people with GAD is not due to its duration, intensity, and uncontrollability, but due to a subjective judgement about the worry being unwanted and excessive.

Worry diary responses were moderately correlated with self-report questionnaire measures of worry and GAD symptoms. The only exception was the number of daily worry 
episodes recorded, which did not correlate with self-report worry or GAD symptom measures. As discussed previously, the effort involved in recording worry episodes may have meant that this measure was sensitive to factors other than worry frequency, and that is why it does not correlate well with other measures of worry. A previous study of WP with high school students (Brosschot \& Van Der Doef, 2006) used a daily worry diary where they asked participants at the end of each day to estimate the number of worry episodes they had that day. This study used the same definition of worry as the present one. Participants' average reported number of daily worry episodes was $27.1(S D=28.3)$ and worry frequency was found to be related to trait worry. Brosschot \& Van Der Doef's sample reported a high frequency of worry, especially considering the sample was unselected. As such, one would predict that if the same measure was given to people with GAD, they would report much more frequent worry. This is in contrast to the average daily episodes logged in the present study: 4.33 in the WP condition and 4.63 in MON. Worry, in GAD, is a high frequency behaviour, which makes it difficult to measure in an accurate, ecological, way without putting undue burden on participants. The estimates in the present study, which were collected using event contingent monitoring, are likely an underestimation of the true worry frequency experienced by the participants. A monitoring protocol in which participants are prompted multiple times throughout the day to report on worry frequency during the intervening period could provide a good balance between accuracy and participant burden and compliance. In summary, the worry record in this study did not appear to provide a valid estimate of worry frequency. However, the association between the other worry diary items (worry duration, control, and intensity) and self-report measures of worry and GAD symptoms is promising and builds confidence in the convergent validity of these worry diary measures. 
This study also provides information about the effects of monitoring worry. A common first step of cognitive-behavioural therapies is to have a person monitor the behaviour they are attempting to change. In the present study, although participants in all conditions showed a reduction in past week worry, it does not appear that monitoring worries has a widespread effect on symptoms related to worry and GAD. This suggests that monitoring worries is not sufficient as an intervention in and of itself, and likely needs to be enhanced, or combined with other treatment strategies in order to translate people's increased awareness of worry into symptom change. Worry outcome journaling is one promising type of enhanced worry monitoring. A study by LaFreniere and Newman (2016) asked undergraduates high in GAD symptoms to monitor the outcomes of their worry daily for 10 days and compared to a control condition where participants were merely recording their thoughts. Participant who monitored the outcomes of their worry showed significantly greater reduction in their self-reported worry compared to those in the control condition. To complete the worry outcome journal participants had to frame their worries as concrete future predictions, rate the likelihood of the worry's occurrence, and later, indicate whether the worry came to pass and how bad the outcome was. The additional steps that outcome journaling requires may facilitate the cognitive reappraisal necessary to make worry monitoring an effective standalone technique.

\section{Strengths and Limitations}

The results of this study should be considered in light of the study's strengths and limitations. One strength of this study is its design. This study reflects one of the most rigorous and well-controlled tests of WP to date. First, the use of diverse outcome measures including cognitive process measures and behavioural tasks provides a more detailed picture about the effects of WP. This is the first study to my knowledge to look at outcome measures beyond 
symptom measures and to use behavioural tasks as outcome measures. Further, the use of daily worry diaries in the active conditions allowed us to examine how worry changed day to day during the course of the intervention, rather than only considering pre to postintervention change in worry.

The use of two control groups is another strength of this study. The active control group was carefully chosen to test whether the specific instructions of WP are helpful above and beyond monitoring worry. This was an outstanding question in the existing literature, and the designs of control groups in other studies have not allowed for clear conclusions to be made about the effects of WP relative to monitoring. The assessment only control group also offers an important comparison for the WP intervention, by demonstrating how the symptoms of participants with GAD can be expected to change over the course of a multivisit study, in the absence of an active intervention. Among other things, this study demonstrated that past week worry and performance on the N-back task improved over the course of the study even without intervention.

One limitation of this study is sample size. An a priori power analysis based on the results of a previous WP study in participants with high trait worry (McGowan \& Behar, 2013) determined that the final sample should be 30 participants per condition to detect a medium effect size. Due to challenges with recruitment and lost data due to drop out or poor compliance, the final sample size fell short of this goal. However, the between-group effect sizes on all outcome measures in the present study were in the small effect to no effect range. Given this, it is likely that if the desired sample size was achieved, the analyses would still not meet significance. Further, the power analysis was conducted for a repeated measures ANOVA, which allowed for the use of the effect size from a previous study (McGowan \& Behar, 2013) to inform the power 
analysis. ANOVA is generally considered to be a more conservative test than HLM, and therefore the predicted sample size was likely an overestimation of the sample that would be needed to detect a medium effect in HLM.

Another limitation of this study is that there was a large amount of missing data for one item of an outcome measure, the PSWQ-PW, due to a programming error. Two scores had to be created: one using the complete items, and one using person-mean imputed data points. Despite this missing data, there are reasons to believe that findings from the PSWQ-PW are reliable. First, the PSWQ-PW measures showed high reliability and demonstrated that there was very little impact on internal reliability from removing the missing item. Second, the overall pattern of results - that there was no significant difference between conditions in worry - converges with other measures of worry, including four diary measures, self-reported GAD symptoms, and a measure of rumination, another form of pathological repetitive thought.

\section{Future Directions}

This study is the first study to examine the effects of WP in people with GAD. Given this, and given the fact that the present findings diverge from the published effects of WP in people with high trait worry, an important future direction is to replicate the present findings. Future studies could build on the present methodology in several ways. It would be important in future studies to continue to find ways to enhance participant motivation and compliance, and to strengthen measures of compliance. It could be useful for experimenters to check in on participants halfway through the intervention to and answer any questions that have arisen. This may more closely mirror how the intervention would be applied in a clinical setting across multiple treatment sessions and help to enhance participant commitment to the intervention. The present study had participants respond to a daily compliance item to indicate whether they had 
completed the worry period. The nature of the intervention makes it difficult to confirm whether participants used the worry period and if they used it according to the intervention's instructions. Future studies could make use of a check in call to ask more questions about compliance. Further, it would be useful to have more information about participants' efforts to disengage from worry. An ecological momentary assessment design where participants are repeatedly prompted to respond to a questionnaire could examine the temporal relationship between efforts to disengage from worry and future worry episodes.

Another future direction for understanding WP would be to test its efficacy in a treatment seeking sample, or to look at its effects within a larger treatment package for GAD. Treatment seeking participants or participants engaged in therapy may have a deeper understanding of the rationale for the intervention or greater motivation that could translate into better engagement with intervention.

\section{Conclusions}

This study sought to determine the efficacy of WP in a sample of people with GAD. WP is commonly recommended as part of treatment packages for GAD and is frequently used by therapists treating pathological worry. However, the efficacy of WP for treating worry in people with GAD has not been established. In the present study, WP did not lead to significant reductions in worry, symptoms of GAD, and associated symptoms and cognitive processes, relative to two control conditions. This study contributes to the understanding of evidence-based treatment for GAD by examining the efficacy of a commonly recommended treatment component. Research on the components that comprise evidence-based treatments for GAD will help to further optimize treatment and will contribute to our understanding of the mechanisms involved in symptom change. 
Table 1

Sample Characteristics Separated by Condition

\begin{tabular}{|c|c|c|c|}
\hline & $\mathrm{WP}(n=22)$ & $\operatorname{MON}(n=23)$ & AX-ONLY $(n=22)$ \\
\hline Age - Mean $(s d)$ & $29.64(10.17)$ & $34.04(13.01)$ & $25.09(8.52)$ \\
\hline \multicolumn{4}{|l|}{ Sex - Frequency $(\%)$} \\
\hline Male & $3(13.6 \%)$ & $2(8.7 \%)$ & $2(9.1 \%)$ \\
\hline Female & $19(86.4 \%)$ & $21(91.3 \%)$ & $20(90.9 \%)$ \\
\hline \multicolumn{4}{|c|}{ Racial/Ethnic Background - Frequency (\%) } \\
\hline European Origin/White & $12(54.5 \%)$ & $12(52.2 \%)$ & $8(36.4 \%)$ \\
\hline $\begin{array}{l}\text { Asian-American/Asian } \\
\text { Origin/Pacific Islander }\end{array}$ & $6(27.3 \%)$ & $5(21.7 \%)$ & $8(36.4 \%)$ \\
\hline Middle Eastern & $1(4.5 \%)$ & $3(13 \%)$ & $1(4.5 \%)$ \\
\hline $\begin{array}{l}\text { African-American/Black/ } \\
\text { Caribbean Origin }\end{array}$ & $1(4.5 \%)$ & $2(8.7 \%)$ & $0(0 \%)$ \\
\hline Latino-a/Hispanic & $1(4.5 \%)$ & $0(0 \%)$ & $1(4.5 \%)$ \\
\hline Bi-Racial/Multiracial & $1(4.5 \%)$ & $1(4.3 \%)$ & $1(4.5 \%)$ \\
\hline Other & $0(0 \%)$ & $0(0 \%)$ & $3(13.6 \%)$ \\
\hline \multicolumn{4}{|l|}{ School Status - Frequency $(\%)$} \\
\hline Full time & $7(31.8 \%)^{\mathrm{a}}$ & $7(30.4 \%)^{\mathrm{a}}$ & $11(50.0 \%)$ \\
\hline Part-time & $1(4.5 \%)^{\mathrm{a}}$ & $3(13.0 \%)^{\mathrm{a}}$ & $3(13.6 \%)$ \\
\hline \multicolumn{4}{|l|}{ Employment Status - Frequency (\%) } \\
\hline Unemployed & $7(31.8 \%)$ & $9(39.1 \%)$ & $7(31.8 \%)$ \\
\hline Employed - full time & $9(40.9 \%)$ & $8(34.8 \%)$ & $5(22.7 \%)$ \\
\hline Employed - part-time & $6(27.3 \%)$ & $6(26.1 \%)$ & $10(45.5 \%)$ \\
\hline \multicolumn{4}{|l|}{ DSM-IV Diagnoses - Frequency (\%) } \\
\hline GAD & $22(100 \%)$ & $23(100 \%)$ & $22(100 \%)$ \\
\hline Comorbid Mood Disorder & $3(13.6 \%)$ & $4(17.4 \%)$ & $3(13.6 \%)$ \\
\hline Comorbid Anxiety Disorder & $4(18.2 \%)$ & $3(13.0 \%)$ & $0(0 \%)$ \\
\hline Other Comorbid Disorder & $2(9.1 \%)$ & $1(4.3 \%)$ & $0(0 \%)$ \\
\hline
\end{tabular}

a item was only applicable to participants currently enrolled in a school program 
Table 2

Internal Consistency for Self-report Measures

\begin{tabular}{lccc}
\hline & \multicolumn{3}{c}{ Cronbach's Alpha } \\
\cline { 2 - 4 } Measure & Baseline & Postintervention & Follow-up \\
\hline PSWQ & .84 & - & - \\
PSWQ-PW 14 item & .86 & .90 & .90 \\
DASS-21 & .89 & .88 & .90 \\
SHCQ & .86 & .88 & .90 \\
ISI & .85 & .88 & .84 \\
CAQ & .94 & .93 & .95 \\
MCQ-Control & .72 & .71 & .84 \\
ACS & .87 & .84 & .84 \\
CAMS-R & .70 & .76 & .78 \\
SMQ & .88 & .83 & .86 \\
NPOQ & .94 & .94 & .94 \\
SPSI-PPO & .78 & .79 & .80 \\
SPSI-RPS & .77 & .76 & .81 \\
SPSI-ICS & .75 & .72 & .89 \\
SPSI-AS & .78 & .82 & .87 \\
RRS & .90 & .92 & .94 \\
\hline
\end{tabular}

Note. PSWQ = Penn State Worry Questionnaire, PSWQ-PW = Penn State Worry Questionnaire - Past Week, DASS-21 = Depression, Anxiety, and Stress Scales - 2, SHCQ = Subjective Health Complaints Questionnaire, ISI = Insomnia Severity Index, CAQ = Cognitive Avoidance Questionnaire, MCQ-Control = Metacognitions Questionnaire-30 Control subscale, ACS = Attentional Control Scale, CAMS-R = Cognitive and Affective Mindfulness Scale-Revised, SMQ = Southampton Mindfulness Questionnaire, NPOQ = Negative Problem Orientation Questionnaire, SPSI-PPO = Social Problem Solving Inventory Positive Problem Orientation Scale; SPSI-RPS = Social Problem Solving Inventory Rational Problem Solving Style Scale, SPSI-ICS = Social Problem Solving Inventory Impulsivity/Carelessness Style Scale, SPSI-AS = Social Problem Solving Inventory Avoidant Style Scale, RRS = Ruminative Response Scale 
Table 3

Means and Standard Deviations for Hypothesis 1Questionnaire Measures at Baseline, Postintervention, and Follow-up Separated by Condition

\begin{tabular}{|c|c|c|c|c|c|c|c|c|c|}
\hline \multirow[b]{2}{*}{ Measure } & \multicolumn{3}{|c|}{ Worry Postponement } & \multicolumn{3}{|c|}{ Monitoring } & \multicolumn{3}{|c|}{ Assessment Only } \\
\hline & $M$ & $S D$ & $\begin{array}{c}\text { Within } \\
\text { Cohen's } d\end{array}$ & $M$ & $S D$ & $\begin{array}{c}\text { Within } \\
\text { Cohen's } d\end{array}$ & $M$ & $S D$ & $\begin{array}{c}\text { Within } \\
\text { Cohen's } d\end{array}$ \\
\hline \multicolumn{10}{|l|}{ PSWQ } \\
\hline Baseline & 65.23 & 7.57 & & 66.78 & 9.46 & & 67.09 & 6.58 & \\
\hline \multicolumn{10}{|l|}{ PSWQ-PW } \\
\hline Baseline & 66.11 & 13.12 & & 71.31 & 10.95 & & 69.50 & 9.88 & \\
\hline Midintervention $^{\mathrm{a}}$ & 58.50 & 13.80 & 0.75 & 62.00 & 11.56 & 1.32 & - & - & \\
\hline Postintervention & 58.07 & 14.67 & 0.07 & 63.75 & 11.28 & 0.29 & 64.51 & 14.37 & 0.53 \\
\hline Follow-up & 59.32 & 15.14 & 0.16 & 62.54 & 11.67 & 0.09 & 64.45 & 14.40 & 0.01 \\
\hline \multicolumn{10}{|l|}{ GAD-Q-IV } \\
\hline Baseline & 10.36 & 1.57 & & 9.79 & 2.80 & & 10.02 & 1.95 & \\
\hline Postintervention & 10.13 & 1.55 & 0.12 & 9.67 & 2.49 & 0.06 & 9.91 & 2.06 & 0.05 \\
\hline Follow-up & 9.84 & 1.77 & 0.15 & 9.69 & 2.97 & 0.01 & 9.03 & 2.89 & 0.59 \\
\hline
\end{tabular}

Note. PSWQ = Penn State Worry Questionnaire, PSWQ-PW = Penn State Worry Questionnaire - Past Week, GAD-Q-IV = Generalized Anxiety Disorder Question for DSM-IV

aarticipants in the Worry Postponement and Monitoring conditions completed the PSWQ-PW one week into the 2-week intervention period. 
Table 4

Compliance to Intervention Instructions in the Worry Postponement and Monitoring Conditions Separated by Condition

\begin{tabular}{|c|c|c|c|c|c|}
\hline \multirow[b]{2}{*}{ Measure } & \multicolumn{2}{|c|}{$\begin{array}{c}\text { Worry Postponement } \\
(n=22) \\
\end{array}$} & \multicolumn{2}{|c|}{ Monitoring $(n=23)$} & \multirow[b]{2}{*}{$\begin{array}{l}\text { Between- } \\
\text { group Effect } \\
(\text { Cohen's } d)\end{array}$} \\
\hline & $M$ & $S D$ & $M$ & $S D$ & \\
\hline $\begin{array}{l}\text { Completed Worry } \\
\text { Records ( } \% \text { of days) }\end{array}$ & 80.84 & 29.52 & 83.23 & 13.38 & 0.11 \\
\hline $\begin{array}{l}\text { Days Completed } \\
\text { Evening } \\
\text { Questionnaire } \\
\text { (\% of days) }\end{array}$ & 90.26 & 10.92 & 88.82 & 11.36 & 0.13 \\
\hline $\begin{array}{l}\text { Days Completed } \\
\text { Morning } \\
\text { Questionnaire (\% of } \\
\text { days) }\end{array}$ & 90.26 & 11.14 & 90.37 & 10.24 & 0.01 \\
\hline $\begin{array}{l}\text { Days Completed } \\
\text { Worry Postponement } \\
\text { (\% of days) }\end{array}$ & 78.57 & 14.11 & - & - & \\
\hline
\end{tabular}


Table 5

Means and Standard Deviations for Hypothesis 2 Questionnaire Measures at Baseline, Postintervention, and Follow-up Separated by Condition

\begin{tabular}{|c|c|c|c|c|c|c|c|c|c|}
\hline \multirow{3}{*}{ Measures } & \multicolumn{3}{|c|}{ Worry Postponement } & \multicolumn{3}{|c|}{ Monitoring } & \multicolumn{3}{|c|}{ Assessment Only } \\
\hline & $M$ & $S D$ & Within & $M$ & $S D$ & Within & $M$ & $S D$ & Within \\
\hline & & & Cohen's $d$ & & & Cohen's $d$ & & & Cohen's $d$ \\
\hline \multicolumn{10}{|l|}{ CAMS-R } \\
\hline Baseline & 23.00 & 2.23 & & 22.65 & 2.64 & & 22.46 & 4.02 & \\
\hline Postintervention & 22.46 & 2.76 & 0.17 & 23.05 & 3.92 & 0.12 & 22.91 & 4.67 & 0.12 \\
\hline Follow-up & 22.71 & 2.70 & 0.11 & 22.48 & 2.93 & 0.19 & 22.59 & 4.01 & 0.12 \\
\hline \multicolumn{10}{|l|}{ SMQ } \\
\hline Baseline & 38.96 & 11.24 & & 39.61 & 15.42 & & 43.00 & 11.70 & \\
\hline Postintervention & 43.00 & 8.04 & 0.41 & 42.64 & 14.76 & 0.36 & 44.18 & 12.17 & 0.17 \\
\hline Follow-up & 39.57 & 10.81 & 0.37 & 41.14 & 14.28 & 0.23 & 45.96 & 12.56 & 0.21 \\
\hline \multicolumn{10}{|l|}{ NPOQ } \\
\hline Baseline & 33.82 & 12.83 & & 33.86 & 11.60 & & 35.46 & 11.90 & \\
\hline Postintervention & 30.46 & 11.39 & 0.50 & 33.73 & 11.77 & 0.03 & 34.55 & 11.77 & 0.11 \\
\hline Follow-up & 32.14 & 12.40 & 0.38 & 34.57 & 12.00 & 0.17 & 33.55 & 11.24 & 0.12 \\
\hline \multicolumn{10}{|l|}{ SPSI-PPO } \\
\hline Baseline & 13.77 & 4.32 & & 16.00 & 4.20 & & 13.96 & 4.74 & \\
\hline Postintervention & 14.09 & 4.99 & 0.13 & 16.36 & 3.59 & 0.04 & 13.68 & 4.85 & 0.12 \\
\hline Follow-up & 14.43 & 5.04 & 0.15 & 16.14 & 3.26 & 0.07 & 13.82 & 5.06 & 0.05 \\
\hline \multicolumn{10}{|l|}{ SPSI-RPS } \\
\hline Baseline & 16.32 & 4.39 & & 17.26 & 4.39 & & 14.82 & 4.27 & \\
\hline Postintervention & 15.32 & 4.43 & 0.31 & 16.82 & 3.78 & 0.09 & 15.00 & 4.64 & 0.06 \\
\hline Follow-up & 15.67 & 4.83 & 0.16 & 16.98 & 4.68 & 0.07 & 14.09 & 4.59 & 0.29 \\
\hline
\end{tabular}


Table 5 Continued

\begin{tabular}{|c|c|c|c|c|c|c|c|c|c|}
\hline \multirow{3}{*}{ Measures } & \multicolumn{3}{|c|}{ Worry Postponement } & \multicolumn{3}{|c|}{ Monitoring } & \multicolumn{3}{|c|}{ Assessment Only } \\
\hline & $M$ & $S D$ & Within & $M$ & $S D$ & Within & $M$ & $S D$ & Within \\
\hline & & & Cohen's $d$ & & & Cohen's $d$ & & & Cohen's $d$ \\
\hline \multicolumn{10}{|l|}{ SPSI-ICS } \\
\hline Baseline & 9.27 & 3.86 & & 9.96 & 4.32 & & 10.18 & 4.07 & \\
\hline Postintervention & 9.23 & 3.02 & 0.02 & 9.14 & 3.69 & 0.37 & 9.23 & 4.08 & 0.33 \\
\hline Baseline & 11.41 & 5.23 & & 9.22 & 3.23 & & 12.64 & 4.22 & \\
\hline Postintervention & 12.64 & 5.39 & 0.45 & 9.59 & 3.35 & 0.10 & 12.68 & 4.89 & 0.01 \\
\hline Follow-up & 12.38 & 5.43 & 0.10 & 10.33 & 4.67 & 0.25 & 12.46 & 5.21 & 0.07 \\
\hline \multicolumn{10}{|l|}{ CAQ } \\
\hline Baseline & 66.36 & 22.62 & & 64.57 & 20.20 & & 63.00 & 19.90 & \\
\hline Baseline & 45.96 & 8.43 & & 48.13 & 11.28 & & 45.73 & 8.07 & \\
\hline Postintervention & 47.18 & 8.19 & 0.24 & 48.96 & 10.36 & 0.37 & 47.23 & 7.74 & 0.28 \\
\hline Follow-up & 47.38 & 8.84 & 0.04 & 47.48 & 10.71 & 0.41 & 46.27 & 6.13 & 0.20 \\
\hline
\end{tabular}

Note. CAMS-R = Cognitive and Affective Mindfulness Scale-Revised, SMQ = Southampton Mindfulness Questionnaire, NPOQ = Negative Problem Orientation Questionnaire, SPSI-PPO = Social Problem Solving Inventory Positive Problem Orientation Scale, SPSI-RPS = Social Problem Solving Inventory Rational Problem Solving Style Scale, SPSI-ICS = Social Problem Solving Inventory Impulsivity/Carelessness Style Scale, SPSI-AS = Social Problem Solving Inventory Avoidant Style Scale, CAQ = Cognitive Avoidance Questionnaire, ACS = Attentional Control Scale. 
Table 6

Means and Standard Deviations for Hypothesis 2 Behavioural Measures at Baseline, Postintervention, and Follow-up Separated by Condition

\begin{tabular}{|c|c|c|c|c|c|c|c|c|c|}
\hline \multirow[b]{2}{*}{ Measures } & \multicolumn{3}{|c|}{ Worry Postponement } & \multicolumn{3}{|c|}{ Monitoring } & \multicolumn{3}{|c|}{ Assessment Only } \\
\hline & $M$ & $S D$ & $\begin{array}{c}\text { Within } \\
\text { Cohen's } d\end{array}$ & $M$ & $S D$ & $\begin{array}{c}\text { Within } \\
\text { Cohen's } d\end{array}$ & $M$ & $S D$ & $\begin{array}{c}\text { Within } \\
\text { Cohen's } d\end{array}$ \\
\hline \multicolumn{10}{|l|}{ N-Back 1-back } \\
\hline Baseline & 0.86 & 0.12 & & 0.90 & 0.08 & & 0.91 & 0.08 & \\
\hline Postintervention & 0.88 & 0.13 & 0.11 & 0.92 & 0.11 & 0.003 & 0.92 & 0.07 & 0.11 \\
\hline Follow-up & 0.90 & 0.07 & 0.35 & 0.95 & 0.05 & 0.43 & 0.90 & 0.08 & 0.21 \\
\hline \multicolumn{10}{|l|}{ N-Back 2-back } \\
\hline Baseline & 0.79 & 0.09 & & 0.77 & 0.12 & & 0.80 & 0.11 & \\
\hline Postintervention & 0.82 & 0.12 & 0.35 & 0.84 & 0.09 & 0.01 & 0.83 & 0.11 & 0.28 \\
\hline Follow-up & 0.84 & 0.10 & 0.21 & 0.86 & 0.09 & 0.16 & 0.84 & 0.11 & 0.17 \\
\hline \multicolumn{10}{|l|}{ N-Back 3-back } \\
\hline Baseline & 0.74 & 0.09 & & 0.73 & 0.11 & & 0.73 & 0.09 & \\
\hline Postintervention & 0.78 & 0.11 & 0.40 & 0.76 & 0.11 & 0.39 & 0.77 & 0.09 & 0.54 \\
\hline Follow-up & 0.78 & 0.07 & 0.03 & 0.80 & 0.08 & 0.42 & 0.78 & 0.11 & 0.16 \\
\hline \multicolumn{10}{|l|}{ RIG-R } \\
\hline Baseline & 38.44 & 14.86 & & 34.51 & 15.32 & & 36.16 & 17.51 & \\
\hline Postintervention & 43.33 & 17.07 & 0.56 & 36.75 & 16.51 & 0.52 & 33.40 & 14.55 & 0.24 \\
\hline Follow-up & 45.13 & 16.80 & 0.26 & 40.84 & 19.17 & 0.34 & 36.20 & 15.62 & 0.35 \\
\hline
\end{tabular}


Table 6 Continued

\begin{tabular}{|c|c|c|c|c|c|c|c|c|c|}
\hline \multirow[b]{2}{*}{ Measures } & \multicolumn{3}{|c|}{ Worry Postponement } & \multicolumn{3}{|c|}{ Monitoring } & \multicolumn{3}{|c|}{ Assessment Only } \\
\hline & $M$ & $S D$ & $\begin{array}{c}\text { Within } \\
\text { Cohen's } d\end{array}$ & $M$ & $S D$ & $\begin{array}{c}\text { Within } \\
\text { Cohen's } d\end{array}$ & $M$ & $S D$ & $\begin{array}{c}\text { Within } \\
\text { Cohen's } d\end{array}$ \\
\hline \multicolumn{10}{|l|}{ RIG-RNG } \\
\hline Baseline & 0.60 & 0.16 & & 0.55 & 0.18 & & 0.58 & 0.17 & \\
\hline Postintervention & 0.63 & 0.18 & 0.29 & 0.58 & 0.17 & 0.32 & 0.54 & 0.16 & 0.39 \\
\hline
\end{tabular}

Note. RIG-R = Random Interval Generation Task - Redundancy score, RIG-RNG = Random Interval Generation Task - Random Number Generation score 
Table 7

Means and Standard Deviations for Hypothesis 4 Measures at Baseline, Postintervention, and Follow-up Separated by Condition

\begin{tabular}{|c|c|c|c|c|c|c|c|c|c|}
\hline \multirow{3}{*}{ Measures } & \multicolumn{3}{|c|}{ Worry Postponement } & \multicolumn{3}{|c|}{ Monitoring } & \multicolumn{3}{|c|}{ Assessment Only } \\
\hline & $M$ & $S D$ & Within & $M$ & $S D$ & Within & $M$ & $S D$ & Within \\
\hline & & & Cohen's $d$ & & & Cohen's $d$ & & & Cohen's $d$ \\
\hline \multicolumn{10}{|l|}{ MCQ-Control } \\
\hline Baseline & 12.91 & 3.65 & & 12.44 & 4.04 & & 12.77 & 3.53 & \\
\hline Postintervention & 12.09 & 3.77 & 0.41 & 12.59 & 4.06 & 0.02 & 12.57 & 3.11 & 0.07 \\
\hline Follow-up & 12.24 & 4.46 & 0.08 & 12.14 & 4.16 & 0.18 & 11.91 & 4.39 & 0.21 \\
\hline \multicolumn{10}{|l|}{ Breathing Focus } \\
\hline \multicolumn{10}{|l|}{ Pre/Post Difference } \\
\hline Baseline & 0.09 & 1.77 & & 0.91 & 2.28 & & 0.05 & 2.40 & \\
\hline Postintervention & 0.27 & 3.28 & 0.06 & 0.91 & 2.22 & $<0.01$ & 0.55 & 1.79 & 0.25 \\
\hline Follow-up & 0.00 & 2.25 & 0.07 & 0.57 & 1.47 & 0.14 & 0.18 & 1.76 & 0.23 \\
\hline \multicolumn{10}{|l|}{ Breathing Focus } \\
\hline \multicolumn{10}{|l|}{ Pre/Post-Negative } \\
\hline Baseline & -0.91 & 1.51 & & -1.17 & 2.15 & & -1.38 & 2.67 & \\
\hline Postintervention & -0.95 & 3.29 & 0.01 & -0.50 & 2.06 & 0.47 & -0.68 & 1.91 & 0.24 \\
\hline Follow-up & -0.58 & 2.46 & 0.10 & -0.95 & 1.88 & 0.16 & -0.64 & 1.47 & 0.03 \\
\hline
\end{tabular}

Note. MCQ-Control = Metacognitions Questionnaire-30 Control subscale

Note. Breathing Focus Pre/Post Difference refers to the difference in total intrusions reported in the preworry breathing period and the postworry breathing period of the breathing focus task. Breathing Focus Pre/Post-Negative refers to the difference in negatively valanced intrusions reported in the preworry breathing period and the postworry breathing period of the breathing focus task 
Table 8

Means and Standard Deviations for Hypothesis 5 Questionnaire Measures at Baseline, Postintervention, and Follow-up Separated by Condition

\begin{tabular}{|c|c|c|c|c|c|c|c|c|c|}
\hline \multirow{3}{*}{ Measures } & \multicolumn{3}{|c|}{ Worry Postponement } & \multicolumn{3}{|c|}{ Monitoring } & \multicolumn{3}{|c|}{ Assessment Only } \\
\hline & $M$ & $S D$ & Within & $M$ & $S D$ & Within & $M$ & $S D$ & Within \\
\hline & & & Cohen's $d$ & & & Cohen's $d$ & & & Cohen's $d$ \\
\hline \multicolumn{10}{|l|}{ DASS-21 Dep } \\
\hline Baseline & 7.86 & 4.58 & & 7.65 & 4.48 & & 9.46 & 5.49 & \\
\hline Postintervention & 6.73 & 5.20 & 0.31 & 6.68 & 4.95 & 0.18 & 10.09 & 5.32 & 0.13 \\
\hline Baseline & 5.77 & 4.79 & & 6.70 & 3.91 & & 6.68 & 4.36 & \\
\hline Postintervention & 5.59 & 4.25 & 0.06 & 5.32 & 3.51 & 0.45 & 6.50 & 4.27 & 0.04 \\
\hline Follow-up & 4.81 & 3.19 & 0.27 & 5.10 & 4.10 & 0.09 & 5.05 & 4.12 & 0.49 \\
\hline \multicolumn{10}{|l|}{ ISI } \\
\hline Baseline & 11.64 & 6.65 & & 11.64 & 5.79 & & 13.82 & 6.48 & \\
\hline Baseline & 22.88 & 13.23 & & 25.16 & 10.39 & & 23.38 & 11.08 & \\
\hline Postintervention & 20.98 & 12.58 & 0.36 & 25.25 & 10.99 & 0.02 & 21.25 & 11.32 & 0.29 \\
\hline Follow-up & 19.69 & 12.92 & 0.27 & 26.25 & 12.47 & 0.18 & 20.50 & 11.86 & 0.15 \\
\hline \multicolumn{10}{|l|}{ RRS } \\
\hline Baseline & 52.64 & 12.41 & & 53.45 & 14.65 & & 53.55 & 11.58 & \\
\hline Postintervention & 52.91 & 13.95 & 0.04 & 53.62 & 14.75 & 0.03 & 52.91 & 12.63 & 0.07 \\
\hline Follow-up & 52.48 & 15.17 & 0.06 & 53.53 & 16.67 & 0.02 & 51.18 & 13.10 & 0.19 \\
\hline
\end{tabular}


Note. PSWQ = Penn State Worry Questionnaire, PSWQ-PW = Penn State Worry Questionnaire - Past Week, GAD-Q-IV = Generalized Anxiety Disorder Question for DSM-IV, CAMS-R = Cognitive and Affective Mindfulness Scale-Revised, SMQ = Southampton Mindfulness Questionnaire, NPOQ = Negative Problem Orientation Questionnaire, SPSI-PPO = Social Problem Solving Inventory Positive Problem Orientation Scale, SPSI-RPS = Social Problem Solving Inventory Rational Problem Solving Style Scale, SPSI-ICS = Social Problem Solving Inventory Impulsivity/Carelessness Style Scale, SPSI-AS = Social Problem Solving Inventory Avoidant Style Scale, CAQ = Cognitive Avoidance Questionnaire, ACS = Attentional Control Scale, MCQ-Control = Metacognitions Questionnaire-30 Control subscale, DASS-21 Dep = Depression, Anxiety, and Stress Scales - 21 Depression Subscale, DASS-21 Anx = The Depression, Anxiety, and Stress Scales - 21 Anxiety Subscale, DASS-21 Stress = The Depression, Anxiety, and Stress Scales 21 Stress Subscale, ISI = Insomnia Severity Index, SHCQ = Subjective Health Complaints Questionnaire, RRS = Ruminative Response Scale 
Table 9

Worry Measures from Daily Worry Questionnaire Separated by Condition

\begin{tabular}{|c|c|c|c|c|c|}
\hline \multirow[b]{2}{*}{ Measure } & \multicolumn{2}{|c|}{$\begin{array}{l}\text { Worry Postponement } \\
\quad(n=22)\end{array}$} & \multicolumn{2}{|c|}{ Monitoring $(n=23)$} & \multirow[b]{2}{*}{$\begin{array}{c}\text { Between-group } \\
\text { Effect Size } \\
\text { (Cohen's } d)\end{array}$} \\
\hline & $M$ & $S D$ & $M$ & $S D$ & \\
\hline $\begin{array}{l}\text { Average Daily } \\
\text { Worry Episodes }\end{array}$ & 4.33 & 3.36 & 4.63 & 2.70 & 0.10 \\
\hline $\begin{array}{l}\text { Average Daily } \\
\text { Worry Duration } \\
\text { (minutes) }\end{array}$ & 102.17 & 51.59 & 92.71 & 52.51 & 0.18 \\
\hline $\begin{array}{l}\text { Average Daily } \\
\text { Worry Intensity }\end{array}$ & 3.26 & 0.60 & 3.27 & 0.65 & 0.02 \\
\hline $\begin{array}{l}\text { Average } \\
\text { Difficulty } \\
\text { Disengaging from } \\
\text { Worry }\end{array}$ & 3.13 & 0.70 & 3.04 & 0.74 & 0.13 \\
\hline
\end{tabular}

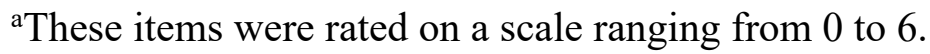


Table 10

Between-group Effect Sizes at Postintervention and Follow-up

\begin{tabular}{|c|c|c|c|c|}
\hline \multirow[b]{2}{*}{ Measure } & \multicolumn{2}{|c|}{$\begin{array}{l}\text { Postintervention Differences } \\
\text { Between-group Effect Size }(d)\end{array}$} & \multicolumn{2}{|c|}{$\begin{array}{l}\text { Follow-up Differences Between- } \\
\text { group Effect Size }(d)\end{array}$} \\
\hline & WP vs. MON & $\begin{array}{l}\text { WP vs. AX- } \\
\text { ONLY }\end{array}$ & WP vs. MON & $\begin{array}{l}\text { WP vs. AX- } \\
\text { ONLY }\end{array}$ \\
\hline PSWQ-PW & 0.44 & 0.44 & 0.24 & 0.35 \\
\hline GAD-Q-IV & 0.23 & 0.12 & 0.06 & 0.34 \\
\hline CAMS-R & 0.18 & 0.12 & 0.08 & 0.04 \\
\hline SMQ & 0.03 & 0.12 & 0.13 & 0.55 \\
\hline NPOQ & 0.28 & 0.35 & 0.20 & 0.12 \\
\hline SPSI-PPO & 0.53 & 0.08 & 0.41 & 0.12 \\
\hline SPSI-RPS & 0.37 & 0.07 & 0.28 & 0.34 \\
\hline SPSI-ICS & 0.03 & $<0.01$ & 0.07 & 0.16 \\
\hline SPSI-AS & 0.70 & 0.01 & 0.41 & 0.02 \\
\hline CAQ & 0.14 & 0.23 & 0.12 & 0.18 \\
\hline ACS & 0.19 & 0.01 & 0.01 & 0.15 \\
\hline MCQ-Control & 0.13 & 0.14 & 0.02 & 0.07 \\
\hline DASS-21 Dep & 0.01 & 0.64 & 0.09 & 0.17 \\
\hline DASS-21 Anx & 0.07 & 0.21 & 0.08 & 0.06 \\
\hline ISI & 0.02 & 0.22 & 0.26 & 0.16 \\
\hline SHCQ & 0.36 & 0.02 & 0.52 & 0.07 \\
\hline RRS & 0.05 & 0.00 & 0.07 & 0.09 \\
\hline Nback 1 back & 0.36 & 0.42 & 0.77 & 0.04 \\
\hline Nback 2 back & 0.20 & 0.02 & 0.13 & 0.03 \\
\hline Nback 3 back & 0.19 & 0.11 & 0.25 & 0.08 \\
\hline RIG-R & 0.39 & 0.63 & 0.24 & 0.55 \\
\hline RIG-RNG & 0.31 & 0.54 & 0.24 & 0.46 \\
\hline $\begin{array}{l}\text { Breathing Focus } \\
\text { Pre/Post }\end{array}$ & 0.23 & 0.11 & 0.31 & 0.09 \\
\hline $\begin{array}{l}\text { Breathing Focus } \\
\text { Negative }\end{array}$ & 0.17 & 0.10 & 0.17 & 0.03 \\
\hline
\end{tabular}


Table 10 Continued

Note. $\mathrm{WP}=$ Worry Postponement, $\mathrm{MON}=$ Monitoring, $\mathrm{AX}-\mathrm{ONLY}=$ Assessment Only

Note. Effect sizes are bolded where the WP group had better scores than the comparison group (i.e., lower scores on the PSWQ-PW, GAD-Q-IV, NPOQ, SPSI-ICS, SPSI-AS, CAQ, MCQControl, DASS-21 Dep, DASS-21 Anx, ISI, SHCQ, RRS, RIG-R, RIG-RNG, and higher scores on the CAMS-R, SMQ, SPSI-PPO, SPSI-RPS, ACS, Nback 1 back, Nback 2 back, Nback 3 back, Breathing Focus Pre/Post Difference, Breathing Focus Pre/Post Negative Intrusions)

Note. PSWQ = PSWQ-PW = Penn State Worry Questionnaire - Past Week, GAD-Q-IV = Generalized Anxiety Disorder Question for DSM-IV, CAMS-R = Cognitive and Affective Mindfulness Scale-Revised, SMQ = Southampton Mindfulness Questionnaire, NPOQ = Negative Problem Orientation Questionnaire, SPSI-PPO = Social Problem Solving Inventory Positive Problem Orientation Scale; SPSI-RPS = Social Problem Solving Inventory Rational Problem Solving Style Scale; SPSI-ICS = Social Problem Solving Inventory Impulsivity/Carelessness Style Scale; SPSI-AS = Social Problem Solving Inventory Avoidant Style Scale; CAQ = Cognitive Avoidance Questionnaire, ACS = Attentional Control Scale, MCQ-Control = Metacognitions Questionnaire-30 Control Subscale, DASS-21 Dep = Depression, Anxiety, and Stress Scales - 21 Depression Subscale, DASS-21 Anx = The Depression, Anxiety, and Stress Scales - 21 Anxiety Subscale, DASS-21 Stress $=$ The Depression, Anxiety, and Stress Scales - 21 Stress Subscale, ISI = Insomnia Severity Index, SHCQ = Subjective Health Complaints Questionnaire, RRS = Ruminative Response Scale, RIG$\mathrm{R}=$ Random Interval Generation Task - Redundancy score, RIG-RNG = Random Interval Generation Task - Random Number Generation score

Note. Breathing Focus Pre/Post refers to the difference in total intrusions reported in the preworry breathing period and the postworry breathing period of the breathing focus task. Breathing Focus Negative refers to the difference in negatively valanced intrusions reported in the preworry breathing period and the postworry breathing period of the breathing focus task. 


\section{Table 11}

Correlations Between Baseline Self-Report Worry Measures and Worry Diary Measures in Worry Postponement and Monitoring Conditions $(n=45)$

\begin{tabular}{lcccc}
\hline Measure & $\begin{array}{c}\text { Average Number } \\
\text { of Daily Worry } \\
\text { Episodes }\end{array}$ & $\begin{array}{c}\text { Average Daily } \\
\text { Worry Duration }\end{array}$ & $\begin{array}{c}\text { Average Worry } \\
\text { Intensity }\end{array}$ & $\begin{array}{c}\text { Average Difficult } \\
\text { Disengaging with } \\
\text { Worry }\end{array}$ \\
\cline { 2 - 5 } PSWQ & $r$ & $r$ & $r$ & $r$ \\
PSWQ-PW & .009 & $.47^{* *}$ & $.37^{*}$ & $.44^{* *}$ \\
GAD-Q-IV & .16 & $.49^{* *}$ & $.41^{* *}$ & $.41^{* *}$ \\
\hline
\end{tabular}

Note. PSWQ = Penn State Worry Questionnaire, PSWQ-PW = Penn State Worry QuestionnairePast Week, GAD-Q-IV = Generalized Anxiety Disorder Questionnaire for DSM-IV.

$*=$ correlation is significant at $p<.05 ; * *=$ correlation is significant at $p<.01$ 
Table 12

Hierarchical Linear Models for Self-Reported Worry and Generalized Anxiety Disorder Symptom Measures in all Three Condition (Hypothesis 1)

\begin{tabular}{llllll}
\hline$b$ & $S E$ & $t$ & $p$ \\
\hline
\end{tabular}

\section{PSWQ-PW 14 item}

Unconditional Growth Model

Intercept 63.35

$-2.21$

1.27

50.01

$<.001 * *$

Time

0.34

$-6.50$

$<.001 * *$

Conditional Growth Model

Intercept

63.73

2.18

29.22

$$
<.001 * *
$$

$-1.47$

0.59

$-2.51$

Time

$-3.58$

3.08

$-1.16$

Worry Postponement

2.34

3.05

0.77

$.02 *$

Monitoring

$-0.84$

0.83

$-1.01$

.25

Time*Worry Postponement

$-1.39$

0.83

$-1.68$

.45

.32

Time*Monitoring

\section{PSWQ-PW 15 item}

Unconditional Growth Model

Intercept

Time

Conditional Growth Model

Intercept
Time
Worry Postponement
Monitoring
Time*Worry Postponement
Time*Monitoring

68.84

$-2.21$

69.38

$-1.62$

$-3.59$

1.87

$-0.72$

$-1.04$
1.40

.38

2.44

0.66

3.45

3.41

0.94

0.93
49.06

$-5.81$

$<.001 * *$

$<.001 * *$

28.47

$-2.45$

$-1.04$

0.55

$-0.77$

$-1.12$
$<.001 * *$

$.02 *$

.30

.58

.45

.27 
Table 12 Continued

$b \quad S E \quad t \quad p$

\section{GAD-Q-IV}

Unconditional Growth Model

$\begin{array}{lcccc}\text { Intercept } & 10.09 & 0.26 & 39.21 & <.001 * * \\ \text { Time } & -0.14 & 0.07 & -1.85 & .07\end{array}$

Conditional Growth Model

$\begin{array}{lcccc}\text { Intercept } & 10.10 & 0.45 & 22.30 & <.001^{* *} \\ \text { Time } & -0.22 & 0.13 & -1.75 & .09 \\ \text { Worry Postponement } & 0.28 & 0.64 & 0.44 & .66 \\ \text { Monitoring } & -0.30 & 0.63 & -0.48 & .64 \\ \text { Time*Worry Postponement } & 0.09 & 0.18 & 0.50 & .62 \\ \text { Time*Monitoring } & 0.17 & 0.18 & 0.95 & .35\end{array}$

Note. PSWQ-PW = Penn State Worry Questionnaire - Past Week, GAD-Q-IV = Generalized Anxiety Disorder Questionnaire for DSM-IV. 
Table 13

Hierarchical Linear Models for Self-Reported Worry in Worry Postponement and Monitoring Conditions (Hypothesis 1)

\begin{tabular}{|c|c|c|c|c|}
\hline & $b$ & $S E$ & $t$ & $p$ \\
\hline \multicolumn{5}{|l|}{ PSWQ-PW 14 item } \\
\hline \multicolumn{5}{|c|}{ Unconditional Growth Model } \\
\hline Intercept & 62.96 & 1.28 & 49.36 & $<.001 * *$ \\
\hline Time & -2.21 & .34 & -6.49 & $<.001 * *$ \\
\hline \multicolumn{5}{|c|}{ Conditional Growth Model } \\
\hline Intercept & 60.94 & 2.00 & 30.33 & $<.001 * *$ \\
\hline Time & -2.64 & .54 & -4.86 & $<.001 * *$ \\
\hline Condition & 2.04 & 1.57 & 1.30 & .20 \\
\hline Time*Condition & .42 & .42 & 1.00 & .32 \\
\hline \multicolumn{5}{|l|}{ PSWQ-PW 15 item } \\
\hline \multicolumn{5}{|c|}{ Unconditional Growth Model } \\
\hline Intercept & 68.37 & 1.41 & 48.49 & $<.001 * *$ \\
\hline Time & -2.21 & .38 & -5.81 & $<.001 * *$ \\
\hline \multicolumn{5}{|c|}{ Conditional Growth Model } \\
\hline Intercept & 66.29 & 2.23 & 29.80 & $<.001 * *$ \\
\hline Time & -2.57 & .61 & -4.24 & $<.001 * *$ \\
\hline Condition & 2.10 & 1.74 & 1.21 & .23 \\
\hline Time*Condition & .36 & .47 & .767 & .45 \\
\hline
\end{tabular}

Note. PSWQ-PW was administered at four time points for both active conditions. 
Table 14

Hierarchical Linear Models for Worry Diary Measures (Hypothesis 1)

$b \quad$ SE $\quad t \quad p$

\section{Worry Duration}

Unconditional Growth Model

Intercept

Time

Conditional Growth Model

Intercept

Time

Condition

Time*Condition
98.68

$-0.77$

96.53

3.90

4.42

$-9.21$

Worry Controllability

Unconditional Growth Model

$$
\text { Intercept }
$$

Time

$-0.09$

$-0.05$

0.06

$-0.08$

Time*Condition

\section{Worry Intensity}

Unconditional Growth Model

Intercept

3.49

Time

$-0.14$

Conditional Growth Model

Intercept

3.48

$-0.14$

0.02

$-0.002$

Time

Condition

Time*Condition
9.69

4.70

13.89

6.68

19.58

9.43

0.14

0.07

0.20

0.10

0.28

0.15

23.28

$-1.24$

$<.001 * *$

.22

$0.58 \quad .56$

0.23

82

.33

16.09

$<.001 * *$

$-0.46$

.65

0.20

.85

$-0.57$

.57

0.19

0.10

0.27 
Table 15

Hierarchical Linear Models for Mindfulness Measures (Hypothesis 2)

$b \quad S E \quad t \quad p$

\section{SHMQ}

Unconditional Growth Model

$\begin{array}{lcccc}\text { Intercept } & 40.66 & 1.57 & 25.98 & <.001 * * \\ \text { Time } & 0.70 & 0.29 & 2.41 & .02 *\end{array}$

Conditional Growth Model

$\begin{array}{lcccc}\text { Intercept } & 42.86 & 2.75 & 15.58 & <.001 * * \\ \text { Time } & 0.77 & 0.51 & 1.51 & .13 \\ \text { Worry Postponement } & -3.48 & 3.89 & -0.90 & .37 \\ \text { Monitoring } & -3.05 & 3.85 & -0.79 & .43 \\ \text { Time*Worry Postponement } & -0.15 & 0.72 & -0.21 & .84 \\ \text { Time*Monitoring } & -0.05 & 0.72 & -0.08 & .94\end{array}$

\section{CAMS-R}

Unconditional Growth Model

Intercept

22.72

0.37

$62.05<.001 * *$

Time

$-0.01$

0.11

$-0.12$

.91

Conditional Growth Model

Intercept

22.50

0.65

34.76

$<.001 * *$

Time

0.08

0.19

0.40

.69

Worry Postponement

0.47

0.92

0.52

.61

Monitoring

0.20

0.91

0.22

.83

Time*Worry Postponement

$-0.22$

0.27

$-0.80$

.42

Time*Monitoring

$-0.05$

0.27

$-0.20$

.84

Note. SHMQ = South Hampton Mindfulness Questionnaire, CAMS-R = Cognitive and Affective Mindfulness Scale-Revised. 
Table 16

Hierarchical Linear Models for Problem Solving and Cognitive Avoidance Measures (Hypothesis 2)

\begin{tabular}{llllll}
\hline & $b$ & $S E$ & $t$ & $p$ \\
\hline
\end{tabular}

NPO

Unconditional Growth Model

Intercept

34.63

1.49

23.20

$<.001 * *$

Time

$-0.57$

0.30

$-1.89$

.06

Conditional Growth Model

Intercept

35.53

2.63

13.50

$<.001 * *$

Time

$-0.51$

0.53

$-0.97$

.34

Worry Postponement

$-1.92$

3.72

$-0.52$

.61

Monitoring

$-0.81$

3.69

$-0.22$

.83

Time*Worry Postponement

$-0.31$

0.75

$-0.42$

.68

Time*Monitoring

0.12

0.75

0.17

.87

\section{SPSI-PPO}

Unconditional Growth Model

Intercept

Time

Conditional Growth Model

Intercept

Time

Worry Postponement

Monitoring

Time*Worry Postponement

Time*Monitoring
14.60

0.05

13.93

$-0.06$

$-0.17$

2.11

0.19

0.14
0.55

0.11

0.94

0.19

1.34

1.32

0.28

0.27

$\begin{array}{cc}26.64 & <.001 * * \\ 0.45 & .65\end{array}$

14.76

$<.001 * *$

$-0.30$

.77

$-0.13$

.90

1.60

.12

0.71

.48

0.50 
Table 16 Continued

$\begin{array}{llll}b & \text { SE } & \text { t }\end{array}$

\section{SPSI-RPS}

Unconditional Growth Model

Intercept

16.17

0.53

30.33

$<.001^{* *}$

Time

$-0.18$

0.11

$-1.58$

.12

Conditional Growth Model

Intercept

14.91

0.92

16.20

$<.001 * *$

Time

$-0.14$

0.20

$-0.70$

.49

Worry Postponement

1.37

1.30

1.05

.30

Monitoring

2.35

1.29

1.82

.07

Time*Worry Postponement

$-0.16$

0.28

$-0.58$

.56

Time*Monitoring

0.05

0.28

0.16

.87

\section{SPSI-ICS}

Unconditional Growth Model

Intercept

9.74

0.48

20.33

$<.001^{* *}$

Time

$-0.08$

0.10

$-0.76$

.45

Conditional Growth Model

Intercept
Time
Worry Postponement
Monitoring
Time*Worry Postponement
Time*Monitoring

10.06

0.85

11.88

$<.001 * *$

$-0.10$

0.18

$-0.57$

.57

$-0.81$

1.20

$-0.67$

.50

$-0.18$

1.19

$-0.16$

.88

0.12

0.25

0.47

.64

$-0.04$

0.25

$-0.18$

.86 
Table 16 Continued

$\begin{array}{llll}b & S E & t & p\end{array}$

\section{SPSI-AS}

Unconditional Growth Model

Intercept

11.07

0.54

20.56

$<.001^{* *}$

Time

0.15

0.10

1.47

.14

Conditional Growth Model

Intercept

12.66

0.90

14.08

$<.001 * *$

Time

$-0.04$

0.18

$-0.19$

.85

Worry Postponement

$-1.18$

1.27

$-0.93$

.36

Monitoring

$-3.49$

1.26

$-2.78$

$.01 * *$

Time*Worry Postponement

0.34

0.26

1.34

.19

Time*Monitoring

0.22

0.25

0.88

.38

\section{CAQ}

Unconditional Growth Model

Intercept

64.73

2.50

$25.87<.001 * *$

Time

$-0.59$

0.55

$-1.07$

.29

Conditional Growth Model

Intercept

63.23

4.42

14.29

$<.001 * *$

Time

$-0.95$

0.97

$-0.98$

.33

Worry Postponement

3.50

6.26

0.56

.58

Monitoring

1.02

6.19

0.17

.87

Time*Worry Postponement

0.21

1.38

0.15

.88

Time*Monitoring

0.86

1.36

0.63

.53

Note. NPO = Negative Problem Orientation Questionnaire, SPSI-PPO = Social Problem Solving Inventory Positive Problem Orientation Scale, SPSI-RPS = Social Problem Solving Inventory Rational Problem Solving Style Scale, SPSI-ICS = Social Problem Solving Inventory Impulsivity/Carelessness Style Scale, SPSI-AS = Social Problem Solving Inventory Avoidant Style Scale, CAQ = Cognitive Avoidance Questionnaire. 
Table 17

Hierarchical Linear Models for Attentional Control Measures (Hypothesis 2)

$b \quad S E \quad t \quad p$

\section{ACS}

Unconditional Growth Model

Intercept

Time

Conditional Growth Model

Intercept

Time

Worry Postponement

Monitoring

Time*Worry Postponement

Time*Monitoring

\section{RIG-R}

Unconditional Growth Model

Intercept

Time

Conditional Growth Model

Intercept

Time

Worry Postponement

Monitoring

Time*Worry Postponement

Time*Monitoring
35.81

46.70

0.23

45.86

0.28

0.13

2.32

0.10

$-0.24$

36.17

1.08

$-0.28$

2.62

$-1.47$

2.15

1.93
1.93

0.44

1.14

0.17

2.00

0.30

2.83

2.80

0.42

0.42

3.41

0.75

4.82

4.77

1.07

1.06
41.07

1.35

$<.001 * *$

.18

$22.91<.001 * *$

$0.93 \quad .36$

$0.05 \quad .96$

0.83

.41

0.24

.81

$-0.56$

.58 
Table 17 Continued

$\begin{array}{llll}b & S E & t & p\end{array}$

\section{RIG-RNG}

Unconditional Growth Model

Intercept

0.58

0.02

28.58

$<.001^{* *}$

Time

0.01

0.004

1.61

.11

Conditional Growth Model

Intercept

0.58

0.04

$16.34<.001^{* *}$

Time

$-0.01$

0.01

$-0.88$

.38

Worry Postponement

0.02

0.05

0.42

.67

Monitoring

$-0.03$

0.05

$-0.58$

.56

Time*Worry Postponement

0.02

0.01

1.85

.07

Time*Monitoring

0.02

0.01

2.02

$.048^{*}$

\section{N-Back 1-back}

Unconditional Growth Model

Intercept

0.89

0.01

75.71

$<.001^{* *}$

Time

0.01

0.003

1.91

.06

Conditional Growth Model

Intercept

Time

Worry Postponement

Monitoring

Time*Worry Postponement

Time*Monitoring
0.91

0.002

$-0.05$

$-0.02$

0.01

0.01
0.02

0.01

0.03

0.03

0.01

0.01
$45.03<.001^{* *}$

$\begin{array}{ll}-0.33 & .74\end{array}$

$\begin{array}{ll}-1.84 & .07\end{array}$

$-0.52 \quad .60$

$1.42 \quad .16$

$1.69 \quad .10$ 
Table 17 Continued

$\begin{array}{llll}b & \text { SE }\end{array}$

N-Back 2-back

Unconditional Growth Model

$\begin{array}{lcccc}\text { Intercept } & 0.79 & 0.01 & 62.87 & <.001^{* *} \\ \text { Time } & 0.02 & 0.003 & 5.14 & <.001^{* *}\end{array}$

Conditional Growth Model

\begin{tabular}{lcccc} 
Intercept & 0.80 & 0.02 & 36.38 & $<.001^{* *}$ \\
Time & 0.01 & 0.01 & 1.97 & .054 \\
Worry Postponement & -0.01 & 0.03 & -0.40 & .69 \\
Monitoring & -0.03 & 0.03 & -0.94 & .35 \\
Time*Worry Postponement & 0.004 & 0.01 & 0.52 & .61 \\
Time*Monitoring & 0.01 & 0.01 & 1.66 & .10 \\
N-Back 3-back & & & & \\
Unconditional Growth Model & & & & \\
Intercept & 0.73 & 0.01 & 61.70 & $<.001^{* *}$ \\
Time & 0.02 & 0.003 & 5.01 & $<.001^{* *}$ \\
onditional Growth Model & & & & \\
Intercept & 0.73 & 0.02 & 34.74 & $<.001^{* *}$ \\
Time & 0.02 & 0.01 & 2.94 & $<.001^{* *}$ \\
Worry Postponement & 0.01 & 0.03 & 0.48 & .63 \\
Monitoring & 0.003 & 0.03 & -0.11 & .92 \\
Time*Worry Postponement & 0.003 & 0.01 & -0.42 & .67 \\
Time*Monitoring & 0.002 & 0.01 & 0.31 & .76 \\
\hline
\end{tabular}

Note. ACS $=$ Attentional Control Scale, RIG-R = Random Interval Generation Task Redundancy Score, RIG-RNG $=$ Random Interval Generation Task Random Number Generation Score. 
Table 18

Hierarchical Linear Models for Frequency of Worry Episodes (Hypothesis 3)

$\begin{array}{llll}b & S E & t\end{array}$

\section{Worry Episodes}

Unconditional Growth Model

Intercept

Time

Conditional Growth Model

Intercept

Time

Condition

Time*Condition

$\begin{array}{cccc}6.01 & 0.69 & 8.74 & <.001^{* *} \\ -1.05 & 0.26 & -4.00 & <.001^{* *}\end{array}$

5.54

$-0.80$

0.90

$-0.48$
0.99

0.38

1.39

0.53
5.57

$-2.09$

0.65

$-0.91$
$<.001 * *$

$.04 *$

.52

.37 
Table 19

Hierarchical Linear Models for Proposed Mediator Variables (Hypothesis 4)

$\begin{array}{llll}b & \quad & t\end{array}$

\section{MCQ-Control}

Unconditional Growth Model

Intercept

Time

Conditional Growth Model

Intercept

Time

Worry Postponement

Monitoring

Time*Worry Postponement

Time*Monitoring
12.72

$-0.18$

12.83

$-0.21$

0.04

$-0.37$

$-0.02$

0.12

\section{Breathing Focus Pre/Post}

Unconditional Growth Model

Intercept

0.39

Time

0.01

0.09

0.08

0.02

0.85

$-0.05$

$-0.15$

Time*Worry Postponement

Time*Monitoring

Conditional Growth Model

Intercept
Time
Worry Postponement
Monitoring
Time*Worry Postponement
Time*Monitoring

0.44

0.10

0.79

0.17

1.11

1.10

0.25

0.25

0.27

0.10

0.46

0.17

0.65

0.65

0.24

0.24
28.59

$-1.78$

$<.001 * *$

.08

$6.30<.001 * *$

$-1.21$

.23

0.04

.97

.74

.94

.64

$-0.34$

$-0.08$

0.48

$p$

\section{.}

0.15


Table 19 Continued

$\begin{array}{llll}b & \text { SE } & t\end{array}$

\section{Breathing Focus Pre/Post Negative}

Unconditional Growth Model

$\begin{array}{lcccc}\text { Intercept } & -1.14 & 0.26 & -4.41 & <.001^{* *} \\ \text { Time } & 0.13 & 0.11 & 1.28 & .21 \\ \text { Conditional Growth Model } & & & & \\ \text { Intercept } & -1.37 & 0.46 & -2.95 & <.01^{* *} \\ \text { Time } & 0.24 & 0.19 & 1.27 & .21 \\ \text { Worry Postponement } & 0.43 & 0.65 & 0.66 & .51 \\ \text { Monitoring } & 0.24 & 0.64 & 0.38 & .71 \\ \text { Time*Worry Postponement } & -0.20 & 0.26 & -0.76 & .45 \\ \text { Time*Monitoring } & -0.11 & 0.26 & -0.43 & .67\end{array}$

Note. MCQ-Control = Metacognitions Questionnaire-30, Breathing Focus Pre/Post Difference refers to the difference in total intrusions reported in the preworry breathing period and the postworry breathing period of the breathing focus task. Breathing Focus Pre/Post Negative Intrusions refers to the difference in negatively valanced intrusions reported in the preworry breathing period and the postworry breathing period of the breathing focus task. 
Table 20

Hierarchical Linear Models for Symptom Measures (Hypothesis 5)

$\begin{array}{llll}b & S E & t\end{array}$

\section{DASS-Depression}

Unconditional Growth Model

Intercept

Time

Conditional Growth Model

Intercept

Time

Worry Postponement

Monitoring

Time*Worry Postponement

Time*Monitoring

DASS-Anxiety

Unconditional Growth Model

Intercept

Time

6.45

$-0.37$

6.82

$-0.37$

$-0.98$

$-0.14$

0.16

$-0.16$

Time*Worry Postponement

Time*Monitoring

Conditional Growth Model

Worry Postponement
0.59

0.15

1.03

0.25

1.46

1.44

0.36

0.36

0.53

0.13

0.93

0.22

1.31

1.30

0.31

0.31
14.14

$-2.32$

9.43

$-1.39$

$-1.28$

$-1.49$

$-0.18$

0.26

$<.001^{* *}$

$.02 *$

12.27

$<.01 * *$

$-2.98$

$<.001$ **

$-0.75$

46

$-0.11$

.91

0.52

.61

$-0.52$

.60 
Table 20 Continued

$\begin{array}{llll}b & S E & t & p\end{array}$

\section{ISI}

Unconditional Growth Model

Intercept

Time

Conditional Growth Model

Intercept

Time

Worry Postponement

Monitoring

Time*Worry Postponement

Time*Monitoring

\section{SHCQ}

Unconditional Growth Model

Intercept

Time

23.82

$-0.57$

1.40

0.29

23.40

$-0.85$

$-0.43$

1.64

$-0.06$

0.92

0.77

0.12

1.36

0.20

1.92

1.90

0.29

0.29

17.04

$-1.97$

.05

Conditional Growth Model

Intercept

2.47

0.49

3.49

3.45

0.70

0.70

1.31
$-0.45$

$<.001^{* *}$

.19

$-1.32$

10.08

$<.001 * *$

.31

.26

.36

.33

.65
Monitoring

Time*Monitoring 
Table 20 Continued

$b \quad S E \quad t \quad p$

\section{RRS}

Unconditional Growth Model

$\begin{array}{lcccc}\text { Intercept } & 53.28 & 1.57 & 33.97 & <.001^{* *} \\ \text { Time } & -0.29 & 0.35 & -0.84 & .40\end{array}$

Conditional Growth Model

$\begin{array}{lcccc}\text { Intercept } & 53.70 & 2.76 & 19.47 & <.001^{* *} \\ \text { Time } & -0.58 & 0.60 & -0.96 & .34 \\ \text { Worry Postponement } & -0.96 & 3.90 & -0.25 & .81 \\ \text { Monitoring } & -0.29 & 3.90 & -0.07 & .94 \\ \text { Time*Worry Postponement } & 0.45 & 0.86 & 0.53 & .60 \\ \text { Time*Monitoring } & 0.42 & 0.86 & 0.48 & .63\end{array}$

Note. DASS-21 Dep = Depression, Anxiety, and Stress Scales -21 Depression Subscale, DASS21 Anx $=$ The Depression, Anxiety, and Stress Scales -21 Anxiety Subscale, DASS-21 Stress = The Depression, Anxiety, and Stress Scales - 21 Stress Subscale, ISI = Insomnia Severity Index, SHCQ = Subjective Health Complaints Questionnaire, RRS = Ruminative Response Scale 
Table 21

Percentage of Participants Achieving Clinically Significant Change on Symptom Measures Separated by Condition

\begin{tabular}{lccc|cc}
\hline \multirow{2}{*}{ Measure } & \multicolumn{3}{c}{ Percent Achieving Clinically Significant } \\
Change & & \\
\cline { 2 - 3 } & Postponement & Monitoring & $\begin{array}{c}\text { Assessment } \\
\text { Only }\end{array}$ & $\chi^{2}$ & $p$ \\
\cline { 2 - 3 } PSWQ-PW & 22.7 & 8.7 & 4.5 & 3.81 & .15 \\
GAD-Q-IV & 4.5 & 13.0 & 13.6 & 1.22 & .54 \\
DASS21-Dep & 0 & 8.7 & 0 & 3.94 & .14 \\
DASS21-Anx & 4.5 & 17.4 & 9.1 & 2.05 & .36 \\
ISI & 4.5 & 0 & 0 & 2.08 & .35 \\
SHCQ & 0 & 0 & 0 & - & - \\
RRS & 0 & 4.3 & 0 & 1.94 & .38 \\
\hline
\end{tabular}

Note. PSWQ-PW = Penn State Worry Questionnaire - Past Week, GAD-Q-IV = Generalized Anxiety Disorder Question for DSM-IV, DASS-21 Dep = Depression, Anxiety, and Stress Scales - 21 Depression Subscale, DASS-21 Anx = The Depression, Anxiety, and Stress Scales 21 Anxiety Subscale, DASS-21 Stress = The Depression, Anxiety, and Stress Scales - 21 Stress Subscale, ISI = Insomnia Severity Index, SHCQ = Subjective Health Complaints Questionnaire, RRS $=$ Ruminative Response Scale 
Table 22

Participant Feedback on Worry Postponement and Monitoring Interventions Separated by Condition

\begin{tabular}{|c|c|c|c|c|}
\hline & $\begin{array}{c}\text { Worry } \\
\text { Postponement } \\
(n=23) \\
\end{array}$ & Monitoring $(n=22)$ & & \\
\hline & Frequency $(\%)$ & Frequency $(\%)$ & $\chi^{2}$ & $p$ \\
\hline $\begin{array}{l}\text { Did not continue using } \\
\text { intervention }\end{array}$ & $10(38.46 \%)$ & $14(63.64 \%)$ & 4.91 & .18 \\
\hline $\begin{array}{l}\text { Continued to use intervention a } \\
\text { few times over the past } 2 \text { weeks }\end{array}$ & $11(42.31) \%$ & $4(18.18 \%)$ & & \\
\hline $\begin{array}{l}\text { Continued to use the } \\
\text { intervention most days over the } \\
\text { past } 2 \text { weeks }\end{array}$ & $1(3.87 \%)$ & $1(4.55 \%)$ & & \\
\hline \multirow{2}{*}{$\begin{array}{l}\text { Continued to use the } \\
\text { intervention daily over the past } 2 \\
\text { weeks }\end{array}$} & $1(3.87 \%)$ & $3(13.64 \%)$ & & \\
\hline & $M(S D)$ & $M(S D)$ & $t$ & $p$ \\
\hline $\begin{array}{l}\text { How helpful was the } \\
\text { intervention to you? }\end{array}$ & $3.54(1.50)$ & $3.86(1.52)$ & -0.72 & .47 \\
\hline $\begin{array}{l}\text { How easy to use was the } \\
\text { intervention to you? }^{\mathrm{a}}\end{array}$ & $4.95(1.81)$ & $6.05(1.35)$ & -2.17 & .04 \\
\hline $\begin{array}{l}\text { How confident would you be in } \\
\text { recommending the intervention } \\
\text { to a friend who experiences } \\
\text { similar problems? } b\end{array}$ & $5.67(1.95)$ & $5.59(2.11)$ & 0.13 & .90 \\
\hline
\end{tabular}

${ }^{\text {a }}$ Rated on a scale from 0 to 7.

${ }^{\mathrm{b}}$ Rated on a scale from1 to 9 . 


\section{Appendix A. Participant Flow Diagram}

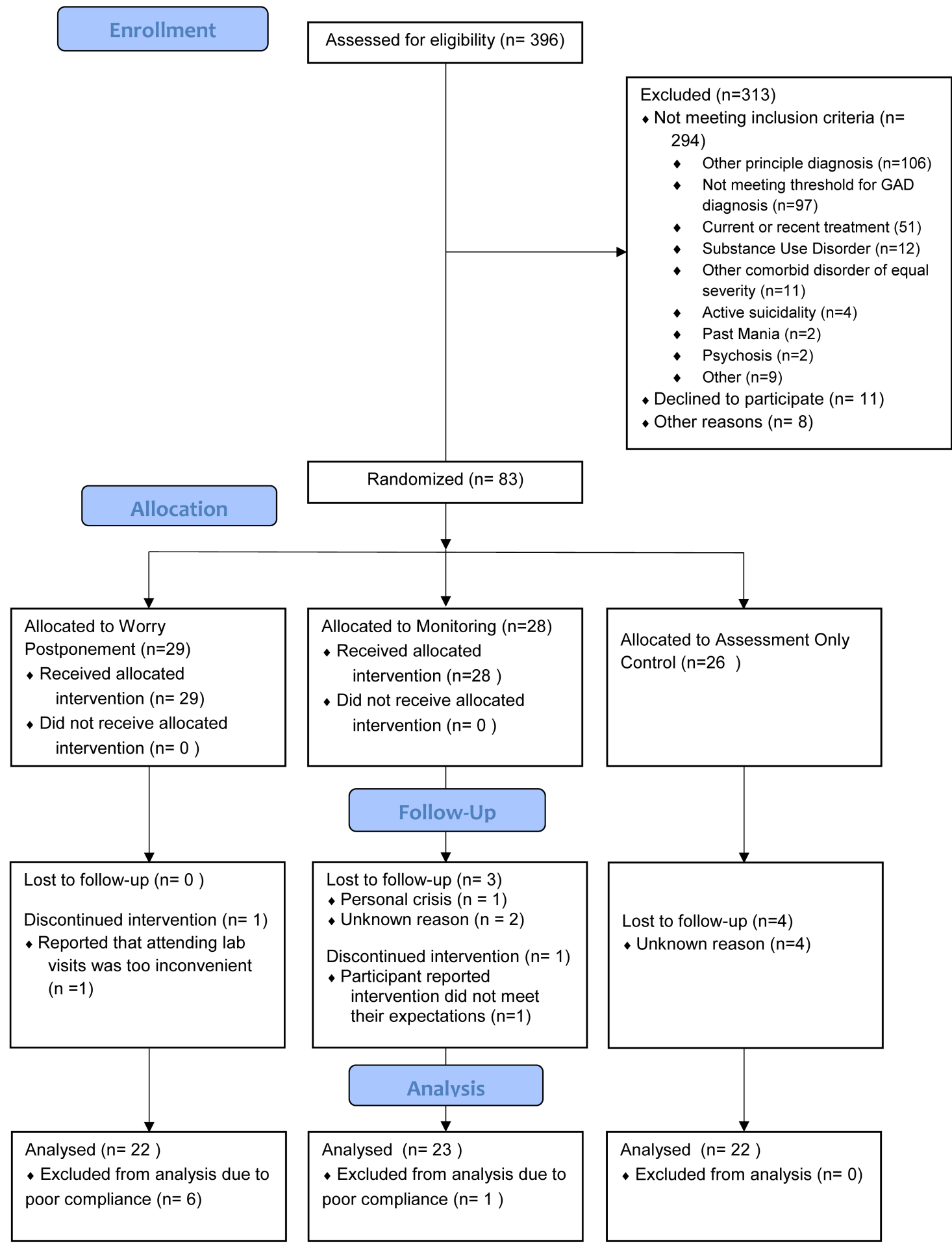


Appendix B. Participant Home Screen of MovisensXS Application

Worry Postponement Condition

$$
\text { 慗 }
$$

งร

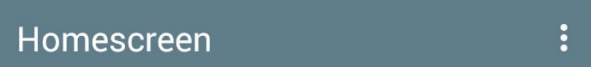

Record your worry

Evening Questionnaire

Morning Questionnaire

Worry Postponement Instructions
Worry Monitoring Condition
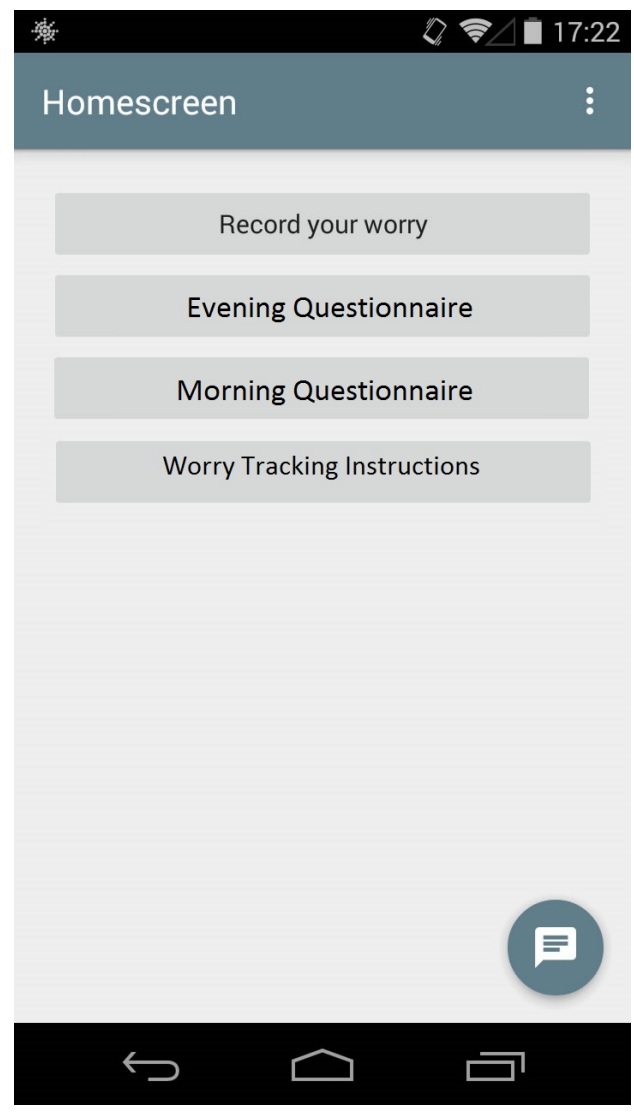
Appendix C. "Record Your Worry" Form

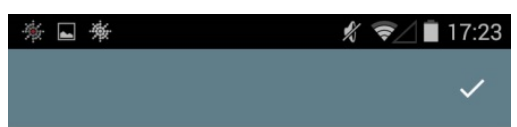

Please write a brief description of what you were worrying about. 
Appendix D. Instructions Forms

Worry Postponement Instructions

Worry Monitoring Instructions

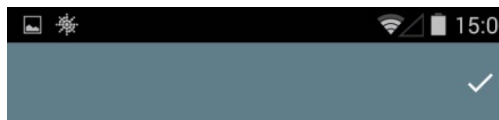

This intervention has four basic instructions:

1. Learn to identify worrisome thoughts and other thoughts that are unnecessary or unpleasant.

Distinguish these from necessary of pleasant thoughts related to the present moment. When you notice a worrisome thought come into your head, record this instance of worry using you e-diary.

2. Establish a half hour worry period to take place at the same time and in the same location each day

3. When you catch yourself worrying, postpone the worry to the worry period and replace it with attending to present-moment experience

4. Make use of the half hour worry period to worry about your concerns and to engage in problem-solving to eliminate those concerns

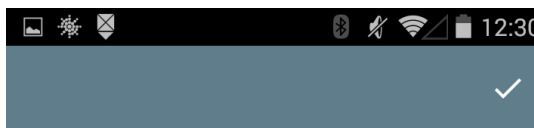

This intervention has four basic instructions:

1. Learn to identify worrisome thoughts and other thoughts that are unnecessary or unpleasant.

Distinguish these from necessary of pleasant thoughts related to the present moment.

2. When you notice a worrisome thought come into your head, record this instance of worry in your ediary.

3. At the end of the day, think back over your day and estimate how many minutes you have spent worrying that day.

4. Continue to monitor and record the frequency and length of your worry episodes over the course of the next two weeks. 
Appendix E. Daily Worry Questionnaire

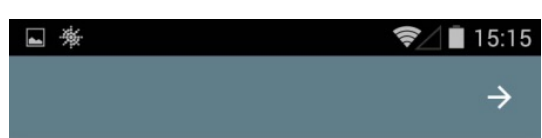

Since completing your Morning Questionnaire, how long did you spend worrying in minutes?

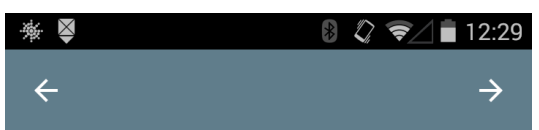

Today when you began worrying, how intense (e.g., strong, extreme, overwhelming) was it?

Not intense at all
\begin{tabular}{|l|l|l|l|l|l|l|}
\multicolumn{4}{c|}{ Very Intense } \\
\hline 0 & 1 & 2 & 3 & 4 & 5 & 6 \\
\hline
\end{tabular}

\section{$\hookleftarrow \square \square$

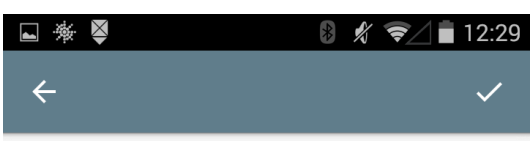

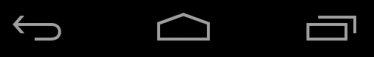

Today, when you began worrying, how difficult was it to stop?

Not at all difficult
\begin{tabular}{|l|l|l|l|l|l|l|}
\hline 0 & 1 & 2 & 3 & 4 & 5 & 6 \\
\hline
\end{tabular}

\section{$\hookleftarrow \square \square$}

Note. This Questionnaire is displayed over three pages. Participants in both the worry postponement and worry monitoring conditions will receive an alarm, alerting them to complete this form once daily in the evening. 
Appendix F. Daily Worry Postponement Questionnaire

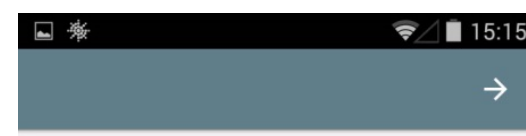

Did you use your worry time today?

Yes

No
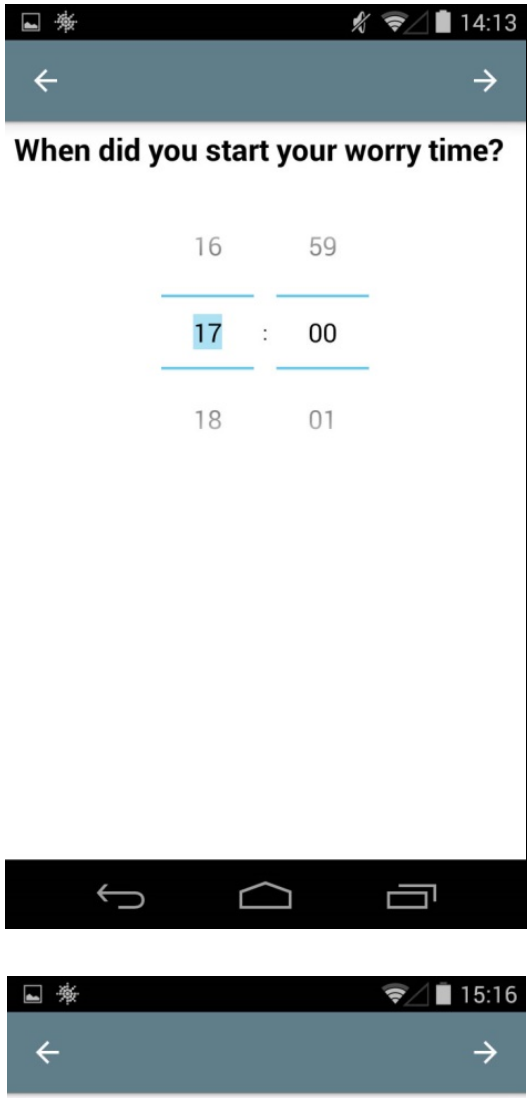

Where did you complete your worry time?
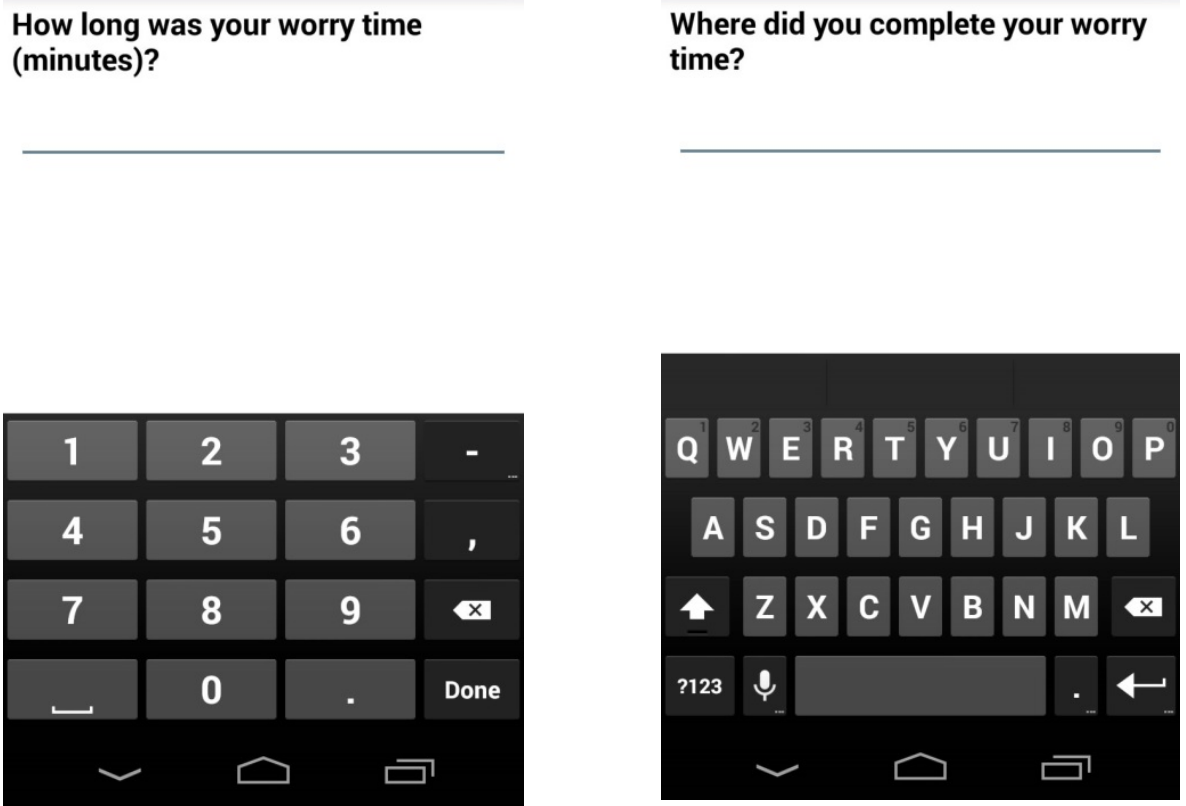

Note. This questionnaire is display over four pages. The questionnaire is displayed only to participants in the worry postponement condition and appears immediately following the daily worry questionnaire items in Appendix E. 


\section{Appendix G. Night Worry Questionnaire}

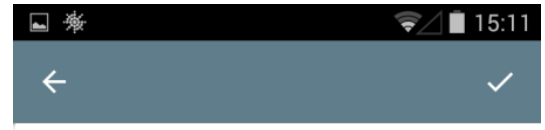

Since you completed your Evening Questionnaire last night, how many minutes did you spend worrying?

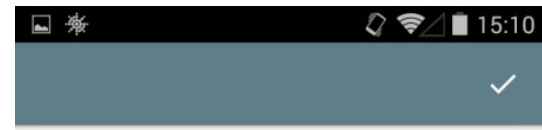

Did you have any worry episodes over night?

$\square$ Yes

$\square$ No

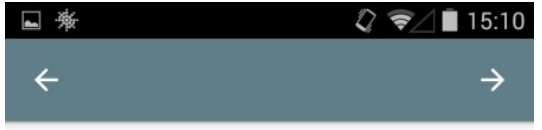

How many episodes of worry did you have overnight?
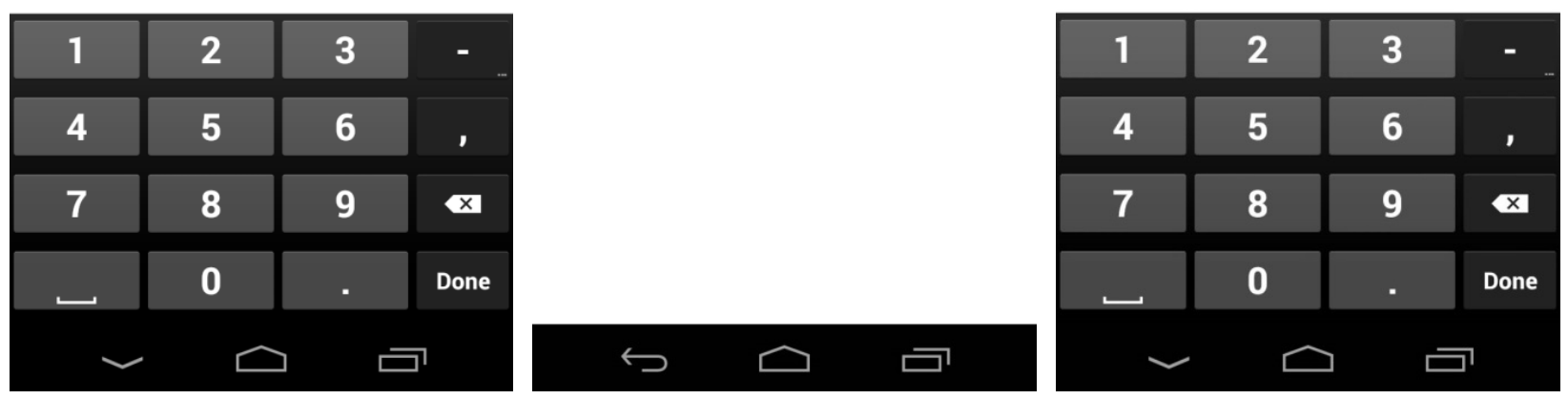

Note. This questionnaire is displayed over three pages. If the first question is answered "no," the subsequent questions are not displayed. Participants in the worry postponement and worry monitoring conditions will receive an alarm in the morning alerting them to complete this form. 


\section{Appendix H. Worry Postponement Instructions}

\section{Worry Postponement Instructions}

Worrying involves thinking about a subject that has or can have negative consequences for yourself, and for which there is no, or not yet, a solution. It often, but not always, consists of a chain of negative thoughts, about the same or different topics, and often concerns something in the future. The thought often takes shape as 'Imagine that . . ' or 'What would happen if . . .?' The same thoughts often return; when you are engaged in worrying it is difficult to stop or control. It definitely occupies your mind, and it is often 'distracting and anxiety-provoking.'

In people who might describe themselves as "worriers," worry often pops into their head frequently throughout the day in any place, time, or situation. When worry occurs throughout the day, it can become associated with many places, times, and situations, such that over time just being exposed to those places, times, and/or situations can come to trigger spontaneous worry. Given this, the goal of this intervention is to reduce the frequency of worry by gradually coming to associate worry with more distinct and specific times and locations, so that only those times and locations come to trigger worry and its associated emotional experiences.

\section{This intervention has four basic instructions:}

1. Learn to identify worrisome thoughts and other thoughts that are unnecessary or unpleasant. Distinguish these from necessary of pleasant thoughts related to the present moment. When you notice a worrisome thought come into your head, record this instance of worry using you e-diary.

2. Establish a half hour worry period to take place at the same time and in the same location each day

3. When you catch yourself worrying, postpone the worry to the worry period and replace it with attending to present-moment experience

4. Make use of the half hour worry period to worry about your concerns and to engage in problem-solving to eliminate those concerns

\section{Tips for setting up your worry period:}

- Pick a 30 minute worry time that is at least 3 hours before your typical bed time to ensure the worry doesn't interfere with your ability to go to sleep

- Pick a location where you will have your worry time each day - for example, it could be at your desk, in a quiet corner of your house, or on the couch

- Try to have your worry period at the same time and location everyday

- Worry as you normally would during your worry period, trying to keep your focus and attention on your worries

- Use this time to problem solve when appropriate 


\section{Appendix I. Worry Monitoring Instructions}

\section{Worry Monitoring Instructions}

Worrying involves thinking about a subject that has or can have negative consequences for yourself, and for which there is no, or not yet, a solution. It often, but not always, consists of a chain of negative thoughts, about the same or different topics, and often concerns something in the future. The thought often takes shape as 'Imagine that ... ' or 'What would happen if . . .?'. The same thoughts often return; when you are engaged in worrying it is difficult to stop or control. It definitely occupies your mind, and it is often 'distracting and anxiety-provoking.'

In people who might describe themselves as "worriers," worry often pops into their head frequently throughout the day in any place, time, or situation. Worry can seem to come about "automatically" or "out of the blue." Because of this, we might not be aware of how much of our day we spend being consumed in our worried thoughts. One of the most important steps in gaining control of your worry is to start bringing attention to when it occurs. You can do this by recognizing and monitoring when worry occurs, how often it occurs, and how much of your day you spend worrying. Through worry monitoring, you will gain helpful knowledge and insight into the patterns influencing your worry.

\section{This intervention has four basic instructions:}

1. Learn to identify worrisome thoughts and other thoughts that are unnecessary or unpleasant. Distinguish these from necessary of pleasant thoughts related to the present moment.

2. When you notice a worrisome thought come into your head, record this instance of worry in your e-diary.

3. At the end of the day, think back over your day and estimate how many minutes you have spent worrying that day.

4. Continue to monitor and record the frequency and length of your worry episodes over the course of the next two weeks. 
Appendix J. Instructions for Completing Phone Forms: Worry Postponement Condition

\section{Worry Tracking Study: Instructions for Completing Your Phone-Based Daily Worry}

\section{Monitoring}

Introduction to the Study: Things to remember

This study is investigating the ways in which learning to recognize and track your worry patterns may affect your experience of worry and anxiety. The study includes you tracking your worry over a two week period using a smartphone application.

You will either be given a Motorola Moto G Smartphone with the MovisensXS application installed to borrow for the duration of the study or have the MovisensXS application installed on your personal smartphone. You will be asked to carry the device with you for the duration of the study. We ask that you respond multiple times a day so that we are able to keep track how these things change, remain the same, or interact over time. You will be provided more specific details about the study when data collection is completed.

Because the study will last for two weeks, it is important that you have the same high level of commitment throughout the study. Each time you make a report, we ask that you are as honest and as thorough as the first time you tried it - otherwise, your answers will not reflect your actual behavior and experiences. We appreciate your interest in the study and hope that you benefit from knowing that you contributed to important research.

\section{Operating the Motorola Moto G Smartphone}

You may be familiar with using Smartphones, but just in case, we have included instructions for a few general functions you will need for this study. All of the functions of the Smartphone not associated with the study are locked, so you will not be able to use the Smartphone for anything but answering questions. This will conserve the battery life of the Smartphone, and thus keep your information safe until we can get it from the Smartphone's memory.

The screen is like a computer screen, and is where all the information will be displayed. You can tap it gently with your finger to select things on screen.

Sometimes you may find yourself in a situation where you do not want the Smartphone alarm to go off. If you desire the turn the alarm completely off, follow these instructions: Press and hold the button on the right side of the phone, select turn off phone. You will press and hold the same button to turn the phone back on. IMPORTANT: Please remember to turn the phone back on.

As you might imagine, these small electronics are quite valuable to us because of the data they carry, but also because they are expensive to purchase and repair. You are therefore held personally responsible for the safekeeping of the device for the entire time you have it. We ask that you take care to treat it gently so that it is not damaged while you use it. You must also return it at the end of your participation in the study. To encourage you to do this, we are having you sign a contract with all of your personal information and the identifier of the specific device you are given for use in the experiment. It is quite important that you bring it back safe and 
sound at the conclusion of your session. You have been scheduled for a session to attend when the study is complete to return the Smartphone and learn more about the study. If you need to reschedule this appointment, please contact the researcher as soon as possible, as there will be other participants waiting to use the device. Devices should be returned to Kathleen Tallon in the Cognition and Psychopathology Lab, 105 Bond St., Room 224 (second floor).

\section{Completing Your Daily Worry Tracking}

There are three forms that you will be asked to complete on a daily basis throughout the next two weeks:

1. Worry Record

2. Evening Questionnaire

3. Morning Questionnaire

Each are described in detail below:

\section{Worry Record:}

- When: This form should be completed throughout the day any time that you catch yourself worrying. Worrying is when you are thinking repetitively about something that is preoccupying and anxiety provoking. You will likely catch yourself worrying multiple times throughout the day. The number of worry records you make might be different each day. We ask that you complete this form, as soon as possible, each time you find yourself worrying. In the evening after completing your Evening Questionnaire and while you are sleeping you are not expected to record you worry. Your experience of evening and nighttime worry will be captured in the Morning Questionnaire.

- Where: You can access this form by opening the movisensXS app and selecting "Worry Record"

- What: This form asks you to provide a brief (i.e., a few words) description of what you were worrying about when you caught yourself worrying (e.g. "money", "argument with roommate", "my health" etc.)

\section{Evening Questionnaire}

- When: This questionnaire should be completed daily, in the early evening. If you do not complete the form, an alarm will go off at 8:00pm reminding you to fill out the form.

- Where: You can access this form by opening the movisensXS app and selecting "Evening Questionnaire"

- What: This questionnaire asks you about your experience of worry throughout the day during the time ranging from when you completed the Morning Questionnaire up until when you fill out this form. This questionnaire also asks you to record some information about your use of worry time that day. Halfway through your two weeks of monitoring there will be 15 additional questions about your experience of worry over the previous week.

\section{Morning Questionnaire}

- When: The questionnaire should be completed daily after you wake up. If you do not complete the form on your own, an alarm will go off at 10:00am to remind you to complete the form.

- Where: You can access this form by opening the movisensXS app and selecting "Morning Questionnaire"

- What: This questionnaire asks you about your experience of worry in the evening and overnight. 


\section{Other information about using movisensXS}

\section{Responding to an Alarm on Motorola Phones}

When an alarm goes off to remind you to complete a form, you can respond to it by swiping downwards with one finger from the top of the screen. You will see the alarm notification. You can choose to "answer" the alarm. This will take you to the questionnaire you need to complete. You can also "delay" the alarm if you would like to complete the questionnaire at a later time. If you chose not to complete the questionnaire you can "dismiss" the alarm.

\section{Silencing the Alarm}

If you do not want to be disturbed, you can silence the phone or set it to vibrate using the volume controls on the right side of the phone. Don't forget to turn the volume back on so that you don't miss an alarm.

\section{Charging the Phone}

Your phone will be fully charged at the beginning of the study. Be sure to regularly charge the phone so that you will be able to use it when necessary. If using the lab phone be sure to always keep it on you so that you can complete your recordings.

\section{Questions or Concerns}

If you ever have any questions or concerns about the intervention or how to complete the phone based monitoring, do not hesitate to contact Kathleen for clarification.

\section{Researcher Contact information:}

\section{Kathleen Tallon}

Tele: $416-979-5000 \times 2188$

Email: caplab@psych.ryerson.ca

\section{Thank you for participating in this study!}


Appendix K. Instructions for Completing Phone Forms: Worry Monitoring Condition

\section{Worry Tracking Study: Instructions for Completing Your Phone-Based Daily Worry}

\section{Monitoring}

Introduction to the Study: Things to remember

This study is investigating the ways in which learning to recognize and track your worry patterns may affect your experience of worry and anxiety. The study includes you tracking your worry over a two week period using a smartphone application.

You will either be given a Motorola Moto G Smartphone with the MovisensXS application installed to borrow for the duration of the study or have the MovisensXS application installed on your personal smartphone. You will be asked to carry the device with you for the duration of the study. We ask that you respond multiple times a day so that we are able to keep track how these things change, remain the same, or interact over time. You will be provided more specific details about the study when data collection is completed.

Because the study will last for two weeks, it is important that you have the same high level of commitment throughout the study. Each time you make a report, we ask that you are as honest and as thorough as the first time you tried it - otherwise, your answers will not reflect your actual behavior and experiences. We appreciate your interest in the study and hope that you benefit from knowing that you contributed to important research.

\section{Operating the Motorola Moto G Smartphone}

You may be familiar with using Smartphones, but just in case, we have included instructions for a few general functions you will need for this study. All of the functions of the Smartphone not associated with the study are locked, so you will not be able to use the Smartphone for anything but answering questions. This will conserve the battery life of the Smartphone, and thus keep your information safe until we can get it from the Smartphone's memory.

The screen is like a computer screen, and is where all the information will be displayed. You can tap it gently with your finger to select things on screen.

Sometimes you may find yourself in a situation where you do not want the Smartphone to signal. If you desire the turn the alarm completely off, follow these instructions: Press and hold the button on the right side of the phone, select turn off phone. You will press and hold the same button to turn the phone back on. IMPORTANT: Please remember to turn the phone back on.

As you might imagine, these small electronics are quite valuable to us because of the data they carry, but also because they are expensive to purchase and repair. You are therefore held personally responsible for the safekeeping of the device for the entire time you have it. We ask that you take care to treat it gently so that it is not damaged while you use it. You must also return it at the end of your participation in the study. To encourage you to do this, we are having you sign a contract with all of your personal information and the identifier of the specific device you are given for use in the experiment. It is quite important that you bring it back safe and 
sound at the conclusion of your session. You have been scheduled for a session to attend when the study is complete to return the Smartphone and learn more about the study. If you need to reschedule this appointment, please contact the researcher as soon as possible, as there will be other participants waiting to use the device. Devices should be returned to Kathleen Tallon in the Cognition and Psychopathology Lab, 105 Bond St., Room 224 (second floor).

\section{Completing Your Daily Worry Tracking}

There are three forms that you will be asked to complete on a daily basis throughout the next two weeks:

4. Worry Record

5. Evening Questionnaire

6. Morning Questionnaire

Each are described in detail below:

\section{Worry Record:}

- When:This form should be completed throughout the day any time that you catch yourself worrying. Worrying is when you are thinking repetitively about something that is preoccupying and anxiety provoking. You will likely catch yourself worrying multiple times throughout the day. The number of worry records you make might be different each day. We ask that you complete this form, as soon as possible, each time you find yourself worrying. In the evening after completing your Evening Questionnaire and while you are sleeping you are not expected to record you worry. Your experience of evening and nighttime worry will be captured in the Morning Questionnaire.

- Where: You can access this form by opening the movisensXS app and selecting "Worry Record"

- What: This form asks you to provide a brief (i.e., a few words) description of what you were worrying about when you caught yourself worrying (e.g. "money", "argument with roommate", "my health" etc.)

\section{Evening Questionnaire}

- When: This questionnaire should be completed daily, in the early evening. If you do not complete the form, an alarm will go off at $8: 00 \mathrm{pm}$ reminding you to fill out the form.

- Where: You can access this form by opening the movisensXS app and selecting "Evening Questionnaire"

- What: This questionnaire asks you about your experience of worry throughout the day during the time ranging from when you completed the Morning Questionnaire up until when you fill out this form. Halfway through your two weeks of monitoring there will be 15 additional questions about your experience of worry over the previous week.

\section{Morning Questionnaire}

- When: The questionnaire should be completed daily after you wake up. If you do not complete the form on your own, an alarm will go off at 10:00am to remind you to complete the form.

- Where: You can access this form by opening the movisensXS app and selecting "Morning Questionnaire" 
- What: This questionnaire asks you about your experience of worry in the evening and overnight.

\section{Other information about using movisensXS}

\section{Responding to an Alarm on Motorola Phones}

When an alarm goes off to remind you to complete a form, you can respond to it by swiping downwards with one finger from the top of the screen. You will see the alarm notification. You can choose to "answer" the alarm. This will take you to the questionnaire you need to complete. You can also "delay" the alarm if you would like to complete the questionnaire at a later time. If you chose not to complete the questionnaire you can "dismiss" the alarm.

\section{Silencing the Alarm}

If you do not want to be disturbed, you can silence the phone or set it to vibrate using the volume controls on the right side of the phone. Don't forget to turn the volume back on so that you don't miss an alarm.

\section{Charging the Phone}

Be sure to regularly charge the phone so that you will be able to use it when necessary. If using the lab phone be sure to always keep it on you so that you can complete your recordings.

\section{Questions or Concerns}

If you ever have any questions or concerns about the intervention or how to complete the phone based monitoring, do not hesitate to contact Kathleen for clarification.

\section{Researcher Contact information:}

\section{Kathleen Tallon}

Tele: 416-979-5000 x 2188

Email: caplab@psych.ryerson.ca

Thank you for participating in this study! 


\section{Appendix L. Worry Postponement Feedback Questionnaire}

In this study you were instructed to use the worry postponement intervention for two weeks. The worry postponement intervention included tracking your worry, postponing worry, and using your daily worry time. We are interested in what your experience was using this intervention. This information will help us understanding how to better improve interventions for worry. Please see the questions below.

1. Did you continue to use worry postponement in the past two weeks?

○ No

- Yes, I used it a few times over the past two weeks

- Yes, I used it most days over the past two weeks

- Yes, I used it daily over the past two weeks

2. How helpful was the worry postponement intervention to you?

$\begin{array}{llllllll}\text { Not at all helpful } 0 & 1 & 2 & 3 & 4 & 5 & 6 & 7 \text { Very helpful }\end{array}$

3. If you found the intervention to be helpful, what did you find helpful about it?

4. Was there any part of worry postponement that you did not like or was unhelpful?

5. How easy to use was the worry postponement intervention?

$\begin{array}{lllllllll}\text { Very difficult to use } & 0 & 1 & 2 & 3 & 4 & 5 & 6 & 7 \text { Very easy to use }\end{array}$

6. How confident would you be in recommending this intervention to a friend who experiences similar problems?

$\begin{array}{llllllllll}\text { Not at all confident } & 1 & 2 & 3 & 4 & 5 & 6 & 7 & 8 & 9 \text { Very confident }\end{array}$

7. When you used your 30-minute worry time, how did you typically use the time (select all that apply):

○ Worrying about concerns that came up during the day

○ Problem solving concerns that came up during the day

- I did not use my worry time

O Other (please specify):

8. Any other comments or feedback on the worry postponement intervention: 


\section{Appendix M. Worry Tracking Feedback Questionnaire}

In this study you were instructed to use the worry tracking intervention for two weeks. The worry tracking intervention included tracking your worry, and completing two daily questionnaires about your worry. We are interested in what your experience was using this intervention. This information will help us understanding how to better improve interventions for worry. Please see the questions below.

1. Did you continue to use worry tracking in the past two weeks?

○ No

- Yes, I used it a few times over the past two weeks

O Yes, I used it most days over the past two weeks

- Yes, I used it daily over the past two weeks

2. How helpful was the worry tracking intervention to you?

$\begin{array}{llllllll}\text { Not at all helpful } 0 & 1 & 2 & 3 & 4 & 5 & 6 & 7 \text { Very helpful }\end{array}$

3. If you found the intervention to be helpful, what did you find helpful about it?

4. Was there any part of worry tracking that you did not like or was unhelpful?

5. How easy to use was the worry tracking intervention?

$\begin{array}{lllllllll}\text { Very easy to use } & 0 & 1 & 2 & 3 & 4 & 5 & 6 & 7\end{array}$

6. How confident would you be in recommending this intervention to a friend who experiences similar problems?

$\begin{array}{llllllllll}\text { Not at all confident } & 1 & 2 & 3 & 4 & 5 & 6 & 7 & 8 & 9 \text { Very confident }\end{array}$

7. Any other comments or feedback on the worry tracking intervention: 
Appendix N. Qualitative Feedback on Worry Postponement Intervention $(n=23)$

\begin{tabular}{|c|c|c|}
\hline & $\begin{array}{l}\text { If you found the intervention to be helpful, what did you } \\
\text { find helpful about it? }\end{array}$ & $\begin{array}{l}\text { Do you have any other comments or feedback on the worry } \\
\text { postponement intervention? }\end{array}$ \\
\hline 1 & $\begin{array}{l}\text { It was good to cut off worrying thoughts and not let them } \\
\text { ruminate whenever possible. I liked having a conscious } \\
\text { moment where I was aware I was having thoughts I } \\
\text { should avoid having. }\end{array}$ & $\begin{array}{l}\text { The hardest part about worry postponement was my varying } \\
\text { schedule. Sometimes, I'd have plans or work in the evening, } \\
\text { which made it very difficult to form a routine. I think this } \\
\text { would work best for someone with a more predictable schedule } \\
\text { than mine. I still like the idea of using it, but know making it a } \\
\text { daily thing is unlikely because of my constantly changing days. }\end{array}$ \\
\hline 2 & $\begin{array}{l}\text { I did not find the intervention too helpful to be honest. } \\
\text { Please see reasons below. }\end{array}$ & $\begin{array}{l}\text { I found it a bit difficult to try and set aside a time and a quiet } \\
\text { place (I live in an apt building (where I wouldn't associate my } \\
\text { worrying thoughts with negativity). Inside my home was an } \\
\text { obvious choice, but for that last reason, I didn't want to do the } \\
\text { intervention there. I still had worrying thoughts despite doing } \\
\text { the intervention, and for me, I feel like distracting myself with } \\
\text { something like, yoga for an hour works better. This way, I am } \\
\text { using my mind and body and doing something positive, } \\
\text { completely focusing on myself. This method of distraction (ex: } \\
\text { going to a spa for a massage), rather than facing my worries } \\
\text { head on is more calming for myself personally. So, treating } \\
\text { myself to something combining the body and mind! }\end{array}$ \\
\hline 3 & $\begin{array}{l}\text { I believe that by assigning time to worry, it allowed me to } \\
\text { focus on the issues fully without being as distracted, as I } \\
\text { would plan around that time. }\end{array}$ & \\
\hline 4 & $\begin{array}{l}\text { It was nice to stave off the worry and get on with the } \\
\text { moment/task at hand. }\end{array}$ & \\
\hline
\end{tabular}




\begin{tabular}{|c|c|c|}
\hline 5 & $\begin{array}{l}\text { I developed the ability to push aside thoughts for a more } \\
\text { appropriate time. }\end{array}$ & I found worry postponement to be very empowering. \\
\hline 6 & $\begin{array}{l}\text { Trying to postpone worrisome thoughts until later so as } \\
\text { not to let it engulf your whole day. Analyzing your actual } \\
\text { worries was helpful as well }\end{array}$ & $\begin{array}{l}\text { Having the postponement 'session' was helpful but also a little } \\
\text { frustrating for me as I found myself thinking about worrying } \\
\text { almost all of the time so it was difficult to dismiss thoughts } \\
\text { until later. }\end{array}$ \\
\hline 7 & $\begin{array}{l}\text { I like the idea of putting off a worry until later to deal } \\
\text { with it. When people tell you to let it go, it's really hard to } \\
\text { because the reason for your worry is that you're trying to } \\
\text { address the problem in a roundabout way. When you } \\
\text { simply try to 'let it go' it doesn't actually help because you } \\
\text { haven't addressed it or solved it yet. I had peace in mind } \\
\text { knowing that I would be able to address it and would } \\
\text { address it later on, which enabled me to fully let the } \\
\text { thought go. }\end{array}$ & $\begin{array}{l}\text { I think it would be better if there was a way to view all the } \\
\text { worries that the person being studied could review. For people } \\
\text { who worry a lot, it's hard to keep track of all of the worries you } \\
\text { have in one day and it would be best to be able to review them } \\
\text { for the problem solving/worry time so that you can see them to } \\
\text { address them one by one. With this worry study,you are able to } \\
\text { record your worries but you cannot see them at the end of the } \\
\text { day. / / In addition, for someone with a very unregulated or } \\
\text { routinized schedule this method was difficult (for example, for } \\
\text { a student). }\end{array}$ \\
\hline 8 & $\begin{array}{l}\text { Could help me be a bit more productive in the moment, } \\
\text { since I would postpone my worrying until a later time }\end{array}$ & \\
\hline 9 & & $\begin{array}{l}\text { I found it difficult to actually postpone the worry. I still tended } \\
\text { to worry about things at that moment and then again during the } \\
\text { postponement time, so it seemed like just extra worrying time. I } \\
\text { also found it difficult to postpone worry to a particular time } \\
\text { every day since my plans change from day to day so sometimes } \\
\text { I would forget or miss it. }\end{array}$ \\
\hline 10 & $\begin{array}{l}\text { I found that pushing my worries and focusing on the } \\
\text { present moment helped to decrease the overall amount of } \\
\text { worrying I did over the course of the day. }\end{array}$ & $\begin{array}{l}\text { I'm glad that I am now more aware of my worries and that my } \\
\text { thoughts seem more organized overall. }\end{array}$ \\
\hline
\end{tabular}




\begin{tabular}{|c|c|c|}
\hline 11 & $\begin{array}{l}\text { I found it helpful because it helped me realize that } \\
\text { worries need not always be in the background and ruin } \\
\text { the day; I can allocate a certain time to deal with them } \\
\text { proactively. }\end{array}$ & $\begin{array}{l}\text { it was difficult to stick to one specific time, because of plans in } \\
\text { the evening. If I recommended the method to someone, I would } \\
\text { recommend they try to do the worry time anytime between five } \\
\text { pm-a few hours before they go to bed. Knowing that there is a } \\
\text { larger time bracket makes it less constraining / / .also for the } \\
\text { question above, used it to problem solve as well / / for question } \\
\text { above: }\end{array}$ \\
\hline 12 & $\begin{array}{l}\text { it helped me keep the worry in perspective? Like, } i \text { would } \\
\text { be able to excuse the worry for the time being and then } \\
\text { revisit it at a later time with a clearer and less anxious } \\
\text { mind }\end{array}$ & nope \\
\hline 13 & $\begin{array}{l}\text { It helped me notice when I was feeling the worry. As I } \\
\text { continued using the intervention, I significantly noticed a } \\
\text { decrease in frequency per day of worrying. It was also } \\
\text { helpful in actually helping with the situations in which I } \\
\text { worried about because during the } 30 \text { minutes, I could } \\
\text { actually do something to better them i.e. applying for new } \\
\text { jobs, and opening e-mails that I was anxious about }\end{array}$ & \\
\hline 14 & $\begin{array}{l}\text { 1) postponing made me aware of the quantity and quality } \\
\text { of the worrying I did / 2) it felt safe to have a parking lot } \\
\text { to put the worrying into / 3) sometimes the worrying } \\
\text { dispersed before I got to worry time / 4) towards the end } \\
\text { of the two weeks the worry time } \\
\text { became more of a worry recollection, sorting and problem } \\
\text { solving time / }\end{array}$ & \\
\hline 15 & & $\begin{array}{l}\text { the app glitched out a few times when I did the study early and } \\
\text { then the alarm went off to do it, it made me do it again instead } \\
\text { of dismissing it for some reason. Also if I accidentally hit the } \\
\text { wrong study then I had to do that one before doing the right }\end{array}$ \\
\hline
\end{tabular}




\begin{tabular}{|c|c|c|}
\hline & & $\begin{array}{l}\text { study (i.e., hitting morning study at night), which meant I } \\
\text { sometimes did two morning studies or two night studies in one } \\
\text { day. Even exiting and closing the app didn't fix that issue, so it's } \\
\text { something to look at going forward perhaps. }\end{array}$ \\
\hline 16 & $\begin{array}{l}\text { It helped me to compartmentalize my worries into a } \\
\text { period where I could readily and actively deal with them } \\
\text { without compromising other priorities. For example, the } \\
\text { time spent worrying during a period became an } \\
\text { opportunity to 'problem-solve', which boosted my } \\
\text { motivation to complete them. It allowed me to accept my } \\
\text { worries as a signal that carried information that I should } \\
\text { somehow be addressing. }\end{array}$ & $\begin{array}{l}\text { It encouraged me to come up with ways to track my worries } \\
\text { using my own smartphone. }\end{array}$ \\
\hline 17 & & $\begin{array}{l}\text { Possibly reduce the time to twenty minutes as it was } \\
\text { discouraging to complete unless I spent the full time, which } \\
\text { was hard to fit in with my night. }\end{array}$ \\
\hline 18 & $\begin{array}{l}\text { / / I found very helpful, specially the part where you } \\
\text { transfer your worries to the worried period. also the } \\
\text { mind constantly worries if that would be money I would } \\
\text { be rich at the end of the two weeks. / }\end{array}$ & $\begin{array}{l}\text { Glad that I had an opportunity in this study, learned quiet a lot } \\
\text { and glad to share. / Thank you. }\end{array}$ \\
\hline 19 & $\begin{array}{l}\text { Surprinsingly What I found VERY helpful was to recored } \\
\text { my worry on the device. It helps me to put some distance } \\
\text { between me and the worry }\end{array}$ & $\begin{array}{l}\text { The worry postponement did not really work for me but the } \\
\text { tracking did tremendously }\end{array}$ \\
\hline 20 & $\begin{array}{l}\text { It felt like getting stuff off my chest before the bed time } \\
\text { hours }\end{array}$ & \\
\hline 21 & $\begin{array}{l}\text { I liked knowing that it was okay for me to worry, just at a } \\
\text { later time. Usually I find that trying to tamp down my } \\
\text { worries just makes them build-up and explode later. But }\end{array}$ & \\
\hline
\end{tabular}




\begin{tabular}{|c|c|c|}
\hline & $\begin{array}{l}\text { releasing my worries at a specified time each day helped } \\
\text { me to release my worries more gradually. }\end{array}$ & \\
\hline 22 & $\begin{array}{l}\text { Because I had to put off my worry till it was my daily } \\
\text { worry time, I did not feel like worrying when it was time. } \\
\text { This just made me not worry during the day because I } \\
\text { would force myself to not worry for the purpose of this } \\
\text { project. }\end{array}$ & $\begin{array}{l}\text { I think this is a very good way to get me to stop worrying } \\
\text { during the day because when worry time came at } 6 \mathrm{pm} \text { I didn't } \\
\text { feel like worrying. So I would go the whole day sometimes } \\
\text { without worrying. On the other hand, I want to worry because } \\
\text { if I don't I start to feel unsettled. Doing this on my own would } \\
\text { be hard because there is no second party involved that I would } \\
\text { we doing this for. I should want to do it to benefit myself but } \\
\text { would just need something to motivate me. }\end{array}$ \\
\hline 23 & $\begin{array}{l}\text { In some ways, shrugging off something is a good way to } \\
\text { get through the day because it makes the worry seem less } \\
\text { important. However, it is hard to get into the habit of } \\
\text { pushing a worry away when you are the type that finds it } \\
\text { hard to stop the worry no matter how hard you try. }\end{array}$ & $\begin{array}{l}\text { Perhaps edit the questions asked if you did not use the worry } \\
\text { time }\end{array}$ \\
\hline
\end{tabular}




\begin{tabular}{|c|c|c|}
\hline & $\begin{array}{l}\text { If you found the intervention to be helpful, what did you } \\
\text { find helpful about it? }\end{array}$ & $\begin{array}{l}\text { Do you have any other comments or feedback on the worry } \\
\text { postponement intervention? }\end{array}$ \\
\hline 1 & $\begin{array}{l}\text { it made me more mindful of my worrying / it made me } \\
\text { think about the difference between chattering mind and } \\
\text { real worry / it made me analyze my worrying to decide if } \\
\text { it was reasonable or unreasonable }\end{array}$ & $\begin{array}{l}\text { Sometimes thinking about tracking my worries worried me. It is } \\
\text { always valuable to draw attention in a non-threatening and non } \\
\text { judgemental way to a behaviour. }\end{array}$ \\
\hline 2 & $\begin{array}{l}\text { It was beneficial to complete the worry tracking because } \\
\text { it would draw my attention to the fact that I was worrying } \\
\text { and would then give me the opportunity to interrupt it. } \\
\text { Also, sometimes the act of writing out and elaborating on } \\
\text { my worry either gave rise to solutions that weren't } \\
\text { previously visible or allowed for enough of an outlet that } \\
\text { I no longer felt the need to continue worrying about that } \\
\text { thing. }\end{array}$ & $\begin{array}{l}\text { The reminders were ideal because I am incredibly forgetful and } \\
\text { I often lose pieces of papers or forget to carry my tracking diary } \\
\text { so having it as a phone app was a great way to ensure I have it } \\
\text { on me and fill it out without excuse. The app was well-designed } \\
\text { and straight-forward to use so that encouraged its use. I } \\
\text { appreciated how it left space for you to elaborate on your } \\
\text { worries where you could write as much or as little as you felt } \\
\text { like in the moment. / / Some suggestions for future studies: (a) } \\
\text { allow for users to see charts and graphs of their worry curves, } \\
\text { (b) collect more data and make it accessible to users, (c) I want } \\
\text { to see what times I worry most and I wish the app would track } \\
\text { the intensity of each worry and make it available to see and } \\
\text { compare, (d) the app should have an algorithm that groups } \\
\text { worries of a similar kind so that users can see what the focus of } \\
\text { most of our worries are, and (e) having an "un-worry" tab that } \\
\text { guides users through a mindfulness technique or contains a } \\
\text { space for users to write their own exercise/technique/positive } \\
\text { message etc. could be a useful tool for interrupting worries in } \\
\text { the moment. }\end{array}$ \\
\hline 3 & $\begin{array}{l}\text { It was helpful having a system in place to help me focus } \\
\text { my worrying and pinpoint the causes of my worrying } \\
\text { each day. }\end{array}$ & $\begin{array}{l}\text { The tracking system was very easy to use - great interface with } \\
\text { no noteable glitches. }\end{array}$ \\
\hline
\end{tabular}




\begin{tabular}{|c|c|c|}
\hline 4 & & $\begin{array}{l}\text { '- I would've liked to be able to go back and review the worries } \\
\text { I had previously recorded. I like to be able to go back and say } \\
\text { to myself, "Okay, well I addressed that worry, I don't need to } \\
\text { worry about it anymore (or, revisit the worry and figure out } \\
\text { what to do about it.) / - The app itself was easy to use, it was } \\
\text { just hard to remember to input worries right when they } \\
\text { happened }\end{array}$ \\
\hline 5 & find out how much time I actually spend worrying & no comment \\
\hline 6 & $\begin{array}{l}\text { It made me reflect on how much wasted time I spent } \\
\text { worrying on tasks vs. actually addressing them and being } \\
\text { productive. It made me realize that worrying was } \\
\text { affecting my sleep. }\end{array}$ & \\
\hline 7 & $\begin{array}{l}\text { to get a sense of the intensity and kind of my worries for } \\
\text { understanding myself }\end{array}$ & \\
\hline 8 & & $\begin{array}{l}\text { It was difficult to track all worries as they came up due to } \\
\text { circumstances (ie. setting where I could not take out the phone, } \\
\text { such as at work) }\end{array}$ \\
\hline 9 & Extra funds for my tuiton fee. & $\begin{array}{l}\text { Thank you for helping me to awake and getting aware of how } \\
\text { worried I've been through the past few months }\end{array}$ \\
\hline 10 & $\begin{array}{l}\text { It is quite helpful to keep track of how often I worry on a } \\
\text { daily basis, and what do I worry about. }\end{array}$ & \\
\hline 11 & & $\begin{array}{l}\text { It felt like tracking my worries actually made me more worried } \\
\text { about things, because I spent more time thinking about them, } \\
\text { and it made me more aware of them by typing them out. }\end{array}$ \\
\hline
\end{tabular}




\begin{tabular}{|c|c|c|}
\hline 12 & $\begin{array}{l}\text { I became more mindful of the occasions when I allowed } \\
\text { worry to set in and used various techniques to mitigate } \\
\text { the effects. My own personal intervention tools. }\end{array}$ & $\begin{array}{l}\text { The tracking exercise did exactly what it was intended to do - I } \\
\text { think the idea of monitoring worrisome thoughts via the app } \\
\text { was a good start. However, I think that once the worrying } \\
\text { thought has been identified there should be a } \\
\text { process/prompt/exercise that enables the user to change } \\
\text { thoughts; to focus on a better feeling thought. :) }\end{array}$ \\
\hline 13 & made me realize how many times a day $\mathrm{i}$ worry & $\begin{array}{l}\text { i dont know how but to not make it forced that I have to } \\
\text { document my worry cause im usually too busy worrying and } \\
\text { forget to write it down }\end{array}$ \\
\hline 14 & $\begin{array}{l}\text { It was a monitoring system, } i \text { found it was very good for } \\
\text { noticing frequency of behaviour patterns. Beeps to } \\
\text { remind me were helpful. }\end{array}$ & $\begin{array}{l}\text { It's a good way to keep track of how often throughout day you } \\
\text { spend time being agitated and concerened about something -- } \\
\text { how much time you are spending thinking about these } \\
\text { concerns, learnt i spend a fair amount of time burdened by these } \\
\text { issues }\end{array}$ \\
\hline 15 & $\begin{array}{l}\text { It was helpful because it interrupted the worry for me to } \\
\text { actually document it, thereby causing me to stop worrying } \\
\text { for that moment. }\end{array}$ & $\begin{array}{l}\text { The app was pretty easy but sometimes it didn't "persist" so I } \\
\text { had to search for it when a worry came up. }\end{array}$ \\
\hline 16 & $\begin{array}{l}\text { It brought attention to topics that I didn't realize I worried } \\
\text { so much about (things/conversations that had already } \\
\text { occurred) }\end{array}$ & $\begin{array}{l}\text { Since I stopped using the worry tracker a little over two weeks } \\
\text { ago, I might have remembered more of the details it had on my } \\
\text { worrying if this questionnaire was asked once I returned the } \\
\text { phone, so it was top of mind. }\end{array}$ \\
\hline 17 & $\begin{array}{l}\text { It made me acknowledge when I was anxious and logging } \\
\text { it made me deal with it immediately, rather than pushing } \\
\text { the anxiousness away or ignoring it. }\end{array}$ & No \\
\hline 18 & $\begin{array}{l}\text { Easier to write down worry thoughts in a designated app } \\
\text { than a general app or physical book. Gave me something } \\
\text { to do related to my anxieties when I can't see my } \\
\text { counselor VS just wallowing in them on my own. }\end{array}$ & \\
\hline
\end{tabular}




\begin{tabular}{|c|c|c|}
\hline & $\begin{array}{l}\text { Obligation to do it (as part of the study) and the } \\
\text { knowledge that they'd be seen by someone and used for } \\
\text { something (ie not just shouting the thoughts into the void) } \\
\text { may have also helped with those points though. }\end{array}$ & \\
\hline 19 & $\begin{array}{l}\text { It allowed me to see what I was worrying when writing it } \\
\text { down, but I also noticed how brief my worries were. }\end{array}$ & $\begin{array}{l}\text { I think tracking the worry is a good idea. However, sometimes I } \\
\text { feel like when you're worrying you forget to track it down. } \\
\text { Maybe, you can have an a range in time that is like } 2-5 \text { hours? } \\
\text { that checks up on you to tell you to jot down your worries } \\
\text { instead of writing it down when you have a worry? }\end{array}$ \\
\hline 20 & $\begin{array}{l}\text { Having a tool to track my worry helped to make me more } \\
\text { conscious of paying attention to my worrying, and the } \\
\text { scales helped to understand how I felt about it. }\end{array}$ & $\begin{array}{l}\text { It was easy to use overall- the only challenge was mentally } \\
\text { remembering to acknowledge the worry and record the details } \\
\text { accordingly }\end{array}$ \\
\hline 21 & $\begin{array}{l}\text { It may be more aware of how often I worried and what I } \\
\text { worried about. Eventually I wondered if the things I } \\
\text { mentioned were really worry worthy, or if I should maybe } \\
\text { ignore it and get over the issue. }\end{array}$ & $\begin{array}{l}\text { The only reason I wouldn't recommend it is, I had times when I } \\
\text { went to sleep early and due to the time being set I had to wake } \\
\text { and fill out the form. I would have rather have filled out the } \\
\text { form when I woke up or before I went to bed. }\end{array}$ \\
\hline
\end{tabular}




\section{References}

Antony, M. M., Bieling, P. J., Cox, B. J., Enns, M. W., \& Swinson, R. P. (1998). Psychometric properties of the 42-item and 21-item versions of the Depression Anxiety Stress Scales in clinical groups and a community sample. Psychological Assessment, 10, 176-181.

American Psychiatric Association. (2013). Diagnostic and statistical manual for mental disorders, $5^{\text {th }}$ ed. Washington, DC: American Psychiatric Association.

Attneave, F. (1959). Applications of information theory to psychology: A summary of basic concepts, methods, and results. Oxford, UK: Henry Holt.

Baddeley, A. D. (1966). The capacity for generating information by randomization. Quarterly Journal of Experimental Psychology, 18, 119-129. doi:10.1080/14640746608400019

Baillargeon, L., Demers, M., \& Ladouceur, R. (1998). Stimulus-control: Nonpharmacologic treatment for insomnia. Canadian Family Physician, 44, 73-79.

Bastien, C. H., Vallières, A., \& Morin, C. M. (2001). Validation of the Insomnia Severity Index as an outcome measure for insomnia research. Sleep Medicine, 2, 297-307. doi:10.1016/S1389-9457(00)00065-4

Beck, A.T., Steer, R.A., \& Brown, G.K. (1996). Manual for the Beck Depression Inventory-II. San Antonio, TX: Psychological Corporation.

Bishop, S. R., Lau, M., Shapiro, S., Carlson, L., Anderson, N. D., Carmody, J., ... \& Devins, G. (2004). Mindfulness: A proposed operational definition. Clinical Psychology: Science and Practice, 11, 230-241. doi:10.1093/clipsy.bph077

Bootzin, R. R. (1972). Stimulus control treatment for insomnia. Proceedings of the Meeting of the American Psychological Association, 7, 395-396. 
Borkovec, T. D. (2006). Applied relaxation and cognitive therapy for pathological worry and generalized anxiety disorder. In G. C. Davey \& A. Wells (Eds.), Worry and its psychological disorders: Theory, assessment and treatment (pp. 273-287). West Sussex, UK: John Wiley and Sons.

Borkovec, T.D., Alcaine, O., \& Behar, E. (2004). Avoidance theory of worry and generalized anxiety disorder. In R.G. Heimberg, C.L. Turk, \& D.S. Mennin (Eds.), Generalized anxiety disorder: Advances in research and practice (pp. 77-108). New York, NY: Guilford Press.

Borkovec, T. D., \& Costello, E. (1993). Efficacy of applied relaxation and cognitive-behavioral therapy in the treatment of generalized anxiety disorder. Journal of Consulting and Clinical Psychology, 61, 611-619. doi:10.1037/0022-006X.61.4.611

Borkovec, T. D., \& Nau, S. D. (1972). Credibility of analogue therapy rationales. Journal of Behavior Therapy and Experimental Psychiatry, 3, 257-260. doi:10.1016/00057916(72)90045-6

Borkovec, T. D., Ray, W. J., \& Stöber, J. (1998). Worry: A cognitive phenomenon intimately linked to affective, physiological, and interpersonal behavioral processes. Cognitive Therapy and Research, 22, 561-576. doi:10.1023/A:1018790003416

Borkovec, T. D., Robinson, E., Pruzinsky, T., \& DePree, J. A. (1983). Preliminary exploration of worry: Some characteristics and processes. Behaviour Research and Therapy, 21, 9-16. doi:10.1016/0005-7967(83)90121-3

Borkovec, T. D., Wilkinson, L., Folensbee, R., \& Lerman, C. (1983). Stimulus control applications to the treatment of worry. Behaviour Research and Therapy, 21, 247-251. doi:10.1016/0005-7967(83)90121-3 
Brosschot, J. F., Gerin, W., \& Thayer, J. F. (2006). The perseverative cognition hypothesis: A review of worry, prolonged stress-related physiological activation, and health. Journal of Psychosomatic Research, 60, 113-124. doi:10.1016/j.jpsychores.2005.06.074

Brosschot, J. F., \& Van Der Doef, M. (2006). Daily worrying and somatic health complaints: Testing the effectiveness of a simple worry reduction intervention. Psychology and Health, 21, 19-31. doi:10.1080/14768320500105346

Brown, T. A., \& Barlow, D. H. (2014). Anxiety and related disorders interview schedule for DSM-5 (ADIS-5) - Lifetime version. New York, NY: Oxford University Press.

Chadwick, P., Hember, M., Symes, J., Peters, E., Kuipers, E., \& Dagnan, D. (2008). Responding mindfully to unpleasant thoughts and images: Reliability and validity of the Southampton Mindfulness Questionnaire (SMQ). British Journal of Clinical Psychology, 47, 451-455. doi:10.1348/014466508X314891

Chesson, A. L., Anderson, W. M., Littner, M., Davila, D., Hartse, K., Johnson, S., ... \& Rafecas, J. (1999). Practice parameters for the nonpharmacologic treatment of chronic insomnia. An American Academy of Sleep Medicine report. Standards of Practice Committee of the American Academy of Sleep Medicine. Sleep, 22, 1128-1133.

Covin, R., Ouimet, A. J., Seeds, P. M., \& Dozois, D. J. (2008). A meta-analysis of CBT for pathological worry among clients with GAD. Journal of Anxiety Disorders, 22, 108-116. doi:10.1016/j.janxdis.2007.01.002

Craigie, M. A., Rees, C. S., Marsh, A., \& Nathan, P. (2008). Mindfulness-based cognitive therapy for generalized anxiety disorder: A preliminary evaluation. Behavioural and Cognitive Psychotherapy, 36, 553-568. doi:10.1017/S135246580800458X 
Crowe, S. F., Matthews, C., \& Walkenhorst, E. (2007). Relationship between worry, anxiety and thought suppression and the components of working memory in a non-clinical sample. Australian Psychologist, 42, 170-177. doi:10.1080/00050060601089462

Cuijpers, P., Sijbrandij, M., Koole, S., Huibers, M., Berking, M., \& Andersson, G. (2014). Psychological treatment of generalized anxiety disorder: A meta-analysis. Clinical Psychology Review, 34, 130-140.

Davey, G. C. (1993). A comparison of three worry questionnaires. Behaviour Research and Therapy, 31, 51-56. doi:10.1016/0005-7967(93)90042-S

Davey, G. C. (1994). Worrying, social problem-solving abilities, and social problem-solving confidence. Behaviour Research and Therapy, 32, 327-330. doi:10.1016/00057967(94)90130-9

Derryberry, D., \& Reed, M. A. (2002). Anxiety-related attentional biases and their regulation by attentional control. Journal of Abnormal Psychology, 111, 225-236. doi:10.1037//0021843X.111.2.225

Devilly, G. J., \& Borkovec, T. D. (2000). Psychometric properties of the credibility/expectancy questionnaire. Journal of Behavior Therapy and Experimental Psychiatry, 31, 73-86. doi:10.1016/S0005-7916(00)00012-4

Dugas, M. J., Brillon, P., Savard, P., Turcotte, J., Gaudet, A., Ladouceur, R., ... \& Gervais, N. J. (2010). A randomized clinical trial of cognitive-behavioral therapy and applied relaxation for adults with generalized anxiety disorder. Behavior Therapy, 41, 46-58. doi:10.1016/j.beth.2008.12.004 
Dugas, M. J., Gagnon, F., Ladouceur, R., \& Freeston, M. H. (1998). Generalized anxiety disorder: A preliminary test of a conceptual model. Behaviour Research and Therapy, 36, 215-226. doi:10.1016/S0005-7967(97)00070-3

Dugas, M. J., Ladouceur, R., Léger, E., Freeston, M. H., Langolis, F., Provencher, M. D., \& Boisvert, J. M. (2003). Group cognitive-behavioral therapy for generalized anxiety disorder: Treatment outcome and long-term follow-up. Journal of Consulting and Clinical Psychology, 71, 821-825. doi:10.1037/0022-006X.71.4.821

Dugas, M. J., Letarte, H., Rhéaume, J., Freeston, M. H., \& Ladouceur, R. (1995). Worry and problem solving: Evidence of a specific relationship. Cognitive Therapy and Research, 19, 109-120. doi:10.1007/BF02229679

D’Zurilla, T. J., Nezu, A. M., \& Maydeu-Olivares, A. (2001). Manual for the Social Problem Solving Inventory-Revised. Toronto, ON: Multi-Health Systems.

Eagleson, C., Hayes, S., Mathews, A., Perman, G., \& Hirsch, C. R. (2016). The power of positive thinking: Pathological worry is reduced by thought replacement in Generalized Anxiety Disorder. Behaviour Research and Therapy, 78, 13-18.

Eriksen, H. R., Ihlebæk, C., \& Ursin, H. (1999). A scoring system for subjective health complaints (SHC). Scandinavian Journal of Public Health, 27, 63-72. doi:10.1177/14034948990270010401

Espie, C. A., Lindsay, W. R., Brooks, D. N., Hood, E. M., \& Turvey, T. (1989). A controlled comparative investigation of psychological treatments for chronic sleep-onset insomnia. Behaviour Research and Therapy, 27, 79-88. doi:10.1016/0005-7967(89)90123-X

Evans, F. J. (1978). Monitoring attention deployment by random number generation index to measure subjective randomness. Bulletin of the Psychonomic Society, 12, 35-38. 
doi:10.3758/BF03329617

Evans, S., Ferrando, S., Findler, M., Stowell, C., Smart, C., \& Haglin, D. (2008). Mindfulnessbased cognitive therapy for generalized anxiety disorder. Journal of Anxiety Disorders, 22, 716-721. doi:10.1016/j.janxdis.2007.07.005

Eysenck, M. W., Derakshan, N., Santos, R., \& Calvo, M. G. (2007). Anxiety and cognitive performance: Attentional control theory. Emotion, 7, 336-353. doi:10.1037/15283542.7 .2 .336

Feldman, G., Hayes, A., Kumar, S., Greeson, J., \& Laurenceau, J. P. (2007). Mindfulness and emotion regulation: The development and initial validation of the Cognitive and Affective Mindfulness Scale-Revised (CAMS-R). Journal of Psychopathology and Behavioral Assessment, 29, 177-190. doi:10.1007/s10862-006-9035-8

Fisher, P. L. (2006). The efficacy of psychological treatments for generalised anxiety disorder. In G. C. Davey \& A. Wells (Eds.), Worry and its psychological disorders: Theory, assessment and treatment (pp. 359-377). West Sussex, UK: John Wiley and Sons.

Foster, C., Startup, H., Potts, L., \& Freeman, D. (2010). A randomised controlled trial of a worry intervention for individuals with persistent persecutory delusions. Journal of Behavior Therapy and Experimental Psychiatry, 41, 45-51. doi:10.1016/j.jbtep.2009.09.001

Gosselin, P., Pelletier, O., \& Ladouceur, R. (2001, July). The Negative Problem Orientation Questionnaire (NPOQ): Development and validation among a non-clinical sample. In Poster session presented at the meeting of the World Congress of Behavioral and Cognitive Therapies. Vancouver, BC. 
Hallion, L. S., Ruscio, A. M., \& Jha, A. P. (2014). Fractionating the role of executive control in control over worry: A preliminary investigation. Behaviour Research and Therapy, 54, 16. doi:10.1016/j.brat.2013.12.002

Hanrahan, F., Field, A. P., Jones, F. W., \& Davey, G. C. (2013). A meta-analysis of cognitive therapy for worry in generalized anxiety disorder. Clinical Psychology Review, 33, 120132. doi:10.1016/j.cpr.2012.10.008

Hayes, A. F. (2006). A primer on multilevel modeling. Human Communication Research, 32, 385-410. doi: 10.1111/j.1468-2958.2006.00281.x

Hayes, A. M., \& Feldman, G. (2004). Clarifying the construct of mindfulness in the context of emotion regulation and the process of change in therapy. Clinical Psychology: Science and Practice, 11, 255-262. doi:10.1093/clipsy.bph080

Hayes, S., Hirsch, C., \& Mathews, A. (2008). Restriction of working memory capacity during worry. Journal of Abnormal Psychology, 117, 712-717. doi:10.1016/j.brat.2009.10.006

Hayes, S., Hirsch, C. R., Krebs, G., \& Mathews, A. (2010). The effects of modifying interpretation bias on worry in generalized anxiety disorder. Behaviour Research and Therapy, 48, 171-178. doi: 10.1016/j.brat.2009.10.006

Hirsch, C. R., Hayes, S., \& Mathews, A. (2009). Looking on the bright side: Accessing benign meanings reduces worry. Journal of Abnormal Psychology, 118, 44-54. doi:10.1037/a0013473

Hirsch, C. R., \& Mathews, A. (2012). A cognitive model of pathological worry. Behaviour Research and Therapy, 50, 636-646. doi:10.1016/j.brat.2012.06.007 
Jacobs, G. D., Benson, H., \& Friedman, R. (1993). Home-based central nervous system assessment of a multifactor behavioral intervention for chronic sleep-onset insomnia. Behavior Therapy, 24, 159-174. doi:10.1016/S0005-7894(05)80261-8

Jacobson, N. S., Follette, W. C., \& Ravenstorf, D. (1984). Psychotherapy outcome research: Methods for reporting variability and evaluating clinical significance. Behavior Therapy, 15, 336-352. doi:10.1016/S0005-7894(84)80002-7

Jellesma, F. C., Verkuil, B., \& Brosschot, J. F. (2009). Postponing worrisome thoughts in children: The effects of a postponement intervention on perseverative thoughts, emotions and somatic complaints. Social Science \& Medicine, 69, 278-284. doi:10.1016/j.socscimed.2009.04.031

Lacks, P., Bertelson, A. D., Gans, L., \& Kunkel, J. (1983). The effectiveness of three behavioral treatments for different degrees of sleep onset insomnia. Behavior Therapy, 14, 593-605. doi:10.1016/S0005-7894(83)80052-5

Ladouceur, R., Dugas, M. J., Freeston, M. H., Léger, E., Gagnon, F., \& Thibodeau, N. (2000). Efficacy of a cognitive-behavioral treatment for generalized anxiety disorder: Evaluation in a controlled clinical trial. Journal of Consulting and Clinical Psychology, 68, 957-964. doi.org/10.1037/0022-006X.68.6.957

Ladouceur, R., Talbot, F., \& Dugas, M. J. (1997). Behavioral expressions of intolerance of uncertainty in worry experimental findings. Behavior Modification, 21, 355-371. doi: $10.1177 / 01454455970213006$

LaFreniere, L. S., \& Newman, M. G. (2016). A brief ecological momentary intervention for generalized anxiety disorder: A randomized controlled trial of the Worry Outcome Journal. Depression and Anxiety, 33, 829-839. doi:10.1002/da.22507 
Leigh, E., \& Hirsch, C. R. (2011). Worry in imagery and verbal form: Effect on residual working memory capacity. Behaviour Research and Therapy, 49, 99-105. doi:10.1016/j.brat.2010.11.005

Lewinsohn, P. M., Biglan, A., \& Zeiss, A. M. (1976). Behavioral treatment of depression. In P. O. Davidson (Ed.), The behavioral management of anxiety, depression and pain (pp. 91146). New York, NY: Brunner/Mazel.

Lewis, C., Pearce, J., \& Bisson, J. I. (2012). Efficacy, cost-effectiveness and acceptability of self-help interventions for anxiety disorders: Systematic review. British Journal of Psychiatry, 200, 15-21. doi:10.1192/bjp.bp.110.084756

Lovibond, P. F., \& Lovibond, S. H. (1995). The structure of negative emotional states: Comparison of the Depression Anxiety Stress Scales (DASS) with the Beck Depression and Anxiety Inventories. Behaviour Research and Therapy, 33, 335-343. doi:10.1016/0005-7967(94)00075-U

Martell, C. R., Addis, M. E., \& Jacobson, N. S. (2001). Depression in context: Strategies for guided action. New York, NY: WW Norton.

Mathews, A., Yiend, J., \& Lawrence, A. D. (2004). Individual differences in the modulation of fear-related brain activation by attentional control. Journal of Cognitive Neuroscience, 16, 1683-1694. doi:10.1162/0898929042947810

Matthews, G., \& Wells, A. (2000). Attention, automaticity, and affective disorder. Behavior Modification, 24, 69-93. doi:10.1177/0145445500241004

McGowan, S. K., \& Behar, E. (2013). A preliminary investigation of stimulus control training for worry: Effects on anxiety and insomnia. Behavior Modification, 37, 90-112. doi:10.1177/0145445512455661 
Meyer, T. J., Miller, M. L., Metzger, R. L., \& Borkovec, T. D. (1990). Development and validation of the Penn State Worry Questionnaire. Behaviour Research and Therapy, 28, 487-495. doi:10.1016/0005-7967(90)90135-6

Miyake, A., Friedman, N. P., Emerson, M. J., Witzki, A. H., Howerter, A., \& Wager, T. D. (2000). The unity and diversity of executive functions and their contributions to complex "frontal lobe" tasks: A latent variable analysis. Cognitive Psychology, 41, 49-100. doi:10.1006/cogp.1999.0734

Molina, S., \& Borkovec, T. D. (1994). The Penn State Worry Questionnaire: Psychometric properties and associated characteristics. In G. C. L. Davey \& F. Tallis (Eds.), Worrying: Perspectives on theory, assessment and treatment (pp. 265-283). Oxford, UK: John Wiley and Sons.

Moore, M. T., Anderson, N. L., Barnes, J. M., Haigh, E. A., \& Fresco, D. M. (2014). Using the GAD-Q-IV to identify generalized anxiety disorder in psychiatric treatment seeking and primary care medical samples. Journal of Anxiety Disorders, 28, 25-30. doi:10.1016/j.janxdis.2013.10.009

Morawetz, D. (1989). Behavioral self-help treatment for insomnia: A controlled evaluation. Behavior Therapy, 20, 365-379. doi:10.1016/S0005-7894(89)80056-5

Morin, C. M., \& Azrin, N. H. (1988). Behavioral and cognitive treatments of geriatric insomnia. Journal of Consulting and Clinical Psychology, 56, 748-753. doi:10.1037/0022006X.56.5.748

Morin, C. M., Culbert, J. P., \& Schwartz, S. M. (1994). Nonpharmacological interventions for insomnia. American Journal of Psychiatry, 151, 1172-1180. doi:10.1176/ajp.151.8.1172

Morin, C. M., Hauri, P. J., Espie, C. A., Spielman, A. J., Buysse, D. J., \& Bootzin, R. R. (1999). 
Nonpharmacologic treatment of chronic insomnia. An American Academy of Sleep Medicine review. Sleep, 22, 1134-1156.

Newman, M. G., \& Borkovec, T. D. (2002). Cognitive behavioral therapy for worry and generalized anxiety disorder. In G. Simos (Ed.), Cognitive behaviour therapy: A guide for the practising clinician (pp. 150-172). New York, NY: Taylor \& Francis.

Newman, M. G., Zuellig, A. R., Kachin, K. E., Constantino, M. J., Przeworski, A., Erickson, T., \& Cashman-McGrath, L. (2002). Preliminary reliability and validity of the Generalized Anxiety Disorder Questionnaire-IV: A revised self-report diagnostic measure of generalized anxiety disorder. Behavior Therapy, 33, 215-233. doi:10.1016/S00057894(02)80026-0

Nolen-Hoeksema, S., \& Morrow, J. (1991). A prospective study of depression and posttraumatic stress symptoms after a natural disaster: The Loma Prieta earthquake. Journal of Personality and Social Psychology, 61, 115-121.

Orsillo, S. M., Roemer, L., \& Barlow, D. H. (2003). Integrating acceptance and mindfulness into existing cognitive-behavioral treatment for GAD: A case study. Cognitive and Behavioral Practice, 10, 222-230. doi:1077-7229/03/222-23051.00/0

Owen, A. M., McMillan, K. M., Laird, A. R., \& Bullmore, E. (2005). N-back working memory paradigm: A meta-analysis of normative functional neuroimaging studies. Human Brain Mapping, 25, 46-59. doi:10.1002/hbm.20131

Robichaud, M., \& Dugas, M. J. (2005a). Negative problem orientation (Part I): Psychometric properties of a new measure. Behaviour Research and Therapy, 43, 391-401. doi:10.1016/j.brat.2004.02.007 
Robichaud, M., \& Dugas, M. J. (2005b). Negative problem orientation (Part II): Construct validity and specificity to worry. Behaviour Research and Therapy, 43, 403-412. doi:10.1016/j.brat.2004.02.008

Robinson, C. M., Klenck, S. C., \& Norton, P. J. (2010). Psychometric properties of the Generalized Anxiety Disorder Questionnaire for DSM-IV among four racial groups. Cognitive Behaviour Therapy, 39, 251-261. doi:10.1080/16506073.2010.486841

Roemer, L., \& Orsillo, S. M. (2002). Expanding our conceptualization of and treatment for generalized anxiety disorder: Integrating mindfulness/acceptance-based approaches with existing cognitive-behavioral models. Clinical Psychology: Science and Practice, 9, 5468. doi:10.1093/clipsy.9.1.54

Roemer, L., \& Orsillo, S. M. (2007). An open trial of an acceptance-based behavior therapy for generalized anxiety disorder. Behavior Therapy, 38, 72-85.

doi:10.1016/j.beth.2006.04.004

Roemer, L., Orsillo, S. M., \& Salters-Pedneault, K. (2008). Efficacy of an acceptance-based behavior therapy for generalized anxiety disorder: Evaluation in a randomized controlled trial. Journal of Consulting and Clinical Psychology, 76, 1083-1089. doi:10.1037/a0012720

Ruscio, A. M., \& Borkovec, T. D. (2004). Experience and appraisal of worry among high worriers with and without generalized anxiety disorder. Behaviour Research and Therapy, 42, 1469-1482. doi:10.1016/j.brat.2003.10.007

Rygh, J. L., \& Sanderson, W. C. (2004). Treating generalized anxiety disorder: Evidence-based strategies, tools, and techniques. New York, NY: Guilford Press.

Sexton, K. A., \& Dugas, M. J. (2008). The cognitive avoidance questionnaire: Validation of the 
English translation. Journal of Anxiety Disorders, 22, 355-370.

doi:10.1016/j.janxdis.2007.04.005

Sheehan, D. V. (2014). Mini International Neuropsychiatric Interview, Version 7.0.0 for DSM-5 (MINI 7.0.0). Jacksonville, FL: Medical Outcomes Systems.

Sheehan, D., Lecrubier, Y., Sheehan, K., Amorim, P. \& Janavs, J. (1998). The Mini-International Neuropsychiatric (MINI): The development and validation of a structured diagnostic psychiatric interview for DSM-IV and ICD-10. Journal of Clinical Psychiatry, 59, 34 57.

Sheehan, D. V., Lecrubier, Y., Harnett Sheehan, K., Janavs, J., Weiller, E., Keskiner, A., ... \& Dunbar, G. C. (1997). The validity of the Mini International Neuropsychiatric Interview (MINI) according to the SCID-P and its reliability. European Psychiatry, 12, 232-241. doi:10.1016/S0924-9338(97)83297-X

Stefanopoulou, E., Hirsch, C. R., Hayes, S., Adlam, A., \& Coker, S. (2014). Are attentional control resources reduced by worry in generalized anxiety disorder? Journal of Abnormal Psychology, 123, 330-335. doi:10.1037/a0036343

Stöber, J. (1998). Worry, problem elaboration and suppression of imagery: The role of concreteness. Behaviour Research and Therapy, 36, 751-756. doi:10.1016/S00057967(98)00027-8

Stöber, J., \& Bittencourt, J. (1998). Weekly assessment of worry: An adaptation of the Penn State Worry Questionnaire for monitoring changes during treatment. Behaviour Research and Therapy, 36, 645-656. doi:10.1016/S0005-7967(98)00031-X 
Stöber, J., \& Borkovec, T. D. (2002). Reduced concreteness of worry in generalized anxiety disorder: Findings from a therapy study. Cognitive Therapy and Research, 26, 89-96. doi:10.1023/A:1013845821848

Szabó, M., \& Lovibond, P. F. (2006). Worry episodes and perceived problem solving: A diarybased approach. Anxiety, Stress, and Coping, 19, 175-187. doi:10.1080/10615800600643562

Szkodny, L. E., Newman, M. G., \& Goldfried, M. R. (2014). Clinical experiences in conducting empirically supported treatments for generalized anxiety disorder. Behavior Therapy, 45, 7-20. doi:10.1016/j.beth.2013.09.009

Tallis, F., Davey, G. C. L., \& Capuzzo, N. (1994). The phenomenology of nonpathological worry: A preliminary investigation. In G. C. L. Davey \& F. Tallis (Eds.), Worrying: Perspectives on theory assessment and treatment (pp. 61-89). West Sussex, UK: John Wiley and Sons.

Tallon, K., Koerner, N., \& Yang, L. (2016). Working memory in Generalized Anxiety Disorder: Effects of verbal and image-based worry and relation to cognitive and emotional processes. Journal of Experimental Psychopathology.

Treynor, W., Gonzalez, R., \& Nolen-Hoeksema, S. (2003). Rumination reconsidered: A psychometric analysis. Cognitive Therapy and Research, 27, 247-259.

Urbaniak, G. C., \& Plous, S. (2013). Research Randomizer (Version 4.0) [Computer software]. Retrieved from http://www.randomizer.org/

Vandierendonck, A., De Vooght, G., \& Van der Goten, K. (1998). Does random time interval generation interfere with working memory executive functions? European Journal of Cognitive Psychology, 10, 413-422. doi:10.1080/713752284 
Verkuil, B., Brosschot, J. F., Korrelboom, K., Reul-Verlaan, R., \& Thayer, J. F. (2011). Pretreatment of worry enhances the effects of stress management therapy: A randomized clinical trial. Psychotherapy and Psychosomatics, 80, 189-190. doi:10.1159/000320328

Verkuil, B., Brosschot, J. F., \& Thayer, J. F. (2007). Capturing worry in daily life: Are trait questionnaires sufficient? Behaviour Research and Therapy, 45, 1835-1844. doi:10.1016/j.brat.2007.02.004

Versluis, A., Verkuil, B., \& Brosschot, J. F. (2016). Reducing worry and subjective health complaints: A randomized trial of an internet-delivered worry postponement intervention. British Journal of Health Psychology, 21, 318-335.

Watkins, E. R. (2008). Constructive and unconstructive repetitive thought. Psychological Bulletin, 134,163-206. doi:10.1037/0033-2909.134.2.163

Wells, A. (1999). A metacognitive model and therapy for generalized anxiety disorder. Clinical Psychology and Psychotherapy, 6, 86-95.

Wells, A. (2006). Metacognitive therapy for worry and generalized anxiety disorder. In G. C. Davey \& A. Wells (Eds.), Worry and its psychological disorders: Theory, assessment and treatment (pp. 179-199). West Sussex, UK: John Wiley and Sons.

Wells, A., \& Cartwright-Hatton, S. (2004). A short form of the metacognitions questionnaire: Properties of the MCQ-30. Behaviour Research and Therapy, 42, 385-396. doi:10.1016/S0005-7967(03)00147-5

Wetherell, J. L., Gatz, M., \& Craske, M. G. (2003). Treatment of generalized anxiety disorder in older adults. Journal of Consulting and Clinical Psychology, 71, 31-40. doi:10.1037/0022-006X.71.1.31 
Wetherell, J. L., Sorrell, J. T., Thorp, S. R., \& Patterson, T. L. (2005). Psychological interventions for late-life anxiety: A review and early lessons from the CALM study. Journal of Geriatric Psychiatry and Neurology, 18, 72-82. doi:10.1177/0891988705276058

Wittchen, H. U. (2002). Generalized anxiety disorder: Prevalence, burden, and cost to society. Depression and Anxiety, 16, 162-171. doi:10.1002/da.10065

Woelk, H., \& Schläfke, S. (2010). A multi-center, double-blind, randomised study of the Lavender oil preparation Silexan in comparison to Lorazepam for generalized anxiety disorder. Phytomedicine, 17, 94-99. doi:10.1016/j.phymed.2009.10.006

Zwart, C. A., \& Lisman, S. A. (1979). Analysis of stimulus control treatment of sleep-onset insomnia. Journal of Consulting and Clinical Psychology, 47, 113. doi:10.1037/0022006X.47.1.11 